\title{
Modular Synthesis of Di- and Trisubstituted Imidazoles from Ketones and Aldehydes: A Route to Kinase Inhibitors
}

\author{
Ian de Toledo, ${ }^{\dagger}$ Thiago A. Grigolo, ${ }^{\dagger}$ James M. Bennett, $^{\dagger}$ Jonathan M. Elkins, $^{\ddagger}, \S$ and Ronaldo A. Pilli* ${ }^{\dagger} \dagger$ \\ ${ }^{\dagger}$ Department of Organic Chemistry, Institute of Chemistry, University of Campinas, UNICAMP, Campinas, São Paulo 13083-970, \\ Brazil \\ ${ }^{\ddagger}$ Structural Genomics Consortium, Nuffield Department of Medicine, University of Oxford, Old Road Campus Research Building, \\ Roosevelt Drive, Oxford OX3 7DQ, United Kingdom \\ ${ }^{\S}$ Structural Genomics Consortium, Departamento de Genética e Evolução, Instituto de Biologia, UNICAMP, Campinas, São Paulo \\ 13083-886, Brazil
}

Supporting Information

ABSTRACT: A one-pot and modular approach to the synthesis of 2,4(5)-disubstituted imidazoles was developed based on ketone oxidation, employing catalytic $\mathrm{HBr}$ and DMSO, followed by imidazole condensation with aldehydes. This methodology afforded twenty-nine disubstituted $\mathrm{NH}$ imidazoles $(23 \%-85 \%$ yield). A three-step synthesis of 20 kinase inhibitors was achieved by employing this oxidationcondensation protocol, followed by bromination and Suzuki coupling in the imidazole ring to yield trisubstituted $\mathrm{NH}$ imidazoles $(23 \%-69 \%$, three steps). This approach was also employed in the synthesis of known inhibitor GSK3037619A.

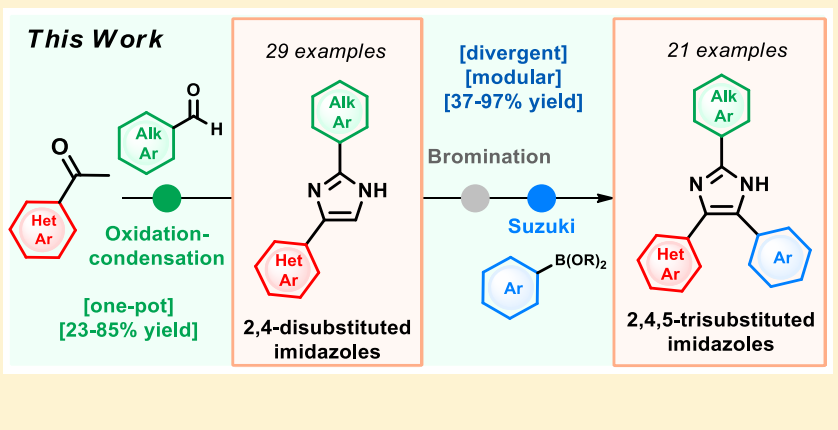

A ccessibility and availability of small organic molecules remains one of the major challenges in the drug discovery process. ${ }^{1}$ Efficient and rapid approaches to access these molecules are highly desirable in order to provide medicinal chemists and chemical biologists the right tools in their scientific endeavors. ${ }^{2}$ In this context, synthetic organic chemistry plays a pivotal role in creating pathways to access these molecules in a short, economic and efficient way from commercially and widely available building blocks. Moreover, the synthetic approach must offer versatility by allowing modular changes in a divergent fashion in order to generate several different molecules from a single precursor.

Substituted imidazoles are one class of such small organic molecules with broad interest, ranging from applications in materials and polymer science ${ }^{3,4}$ to their use as ionic liquids, ${ }^{5}$ and as therapeutic agents ${ }^{6}$ and bioactive molecules such as the marine alkaloids Nortopsentins $\mathrm{A}-\mathrm{C}^{7}$ (Figure 1). Methods to access these scaffolds have been intensely explored and can be roughly divided into two approaches. The first approach involves the formation of the imidazole ring from suitable precursors, ${ }^{8}$ while the second involves the functionalization of a preformed imidazole ring. ${ }^{9}$ Combinations of both approaches can also be employed to efficiently assemble substituted imidazoles. $^{10-14}$

In our search for selective and potent inhibitors of the kinase STK $10,{ }^{15}$ which is a serine-threonine kinase important due to its role in lymphocyte migration, ${ }^{16-19}$ we were challenged with the task of providing an efficient, modular and divergent synthetic route for rapid evaluation of the structure-activity

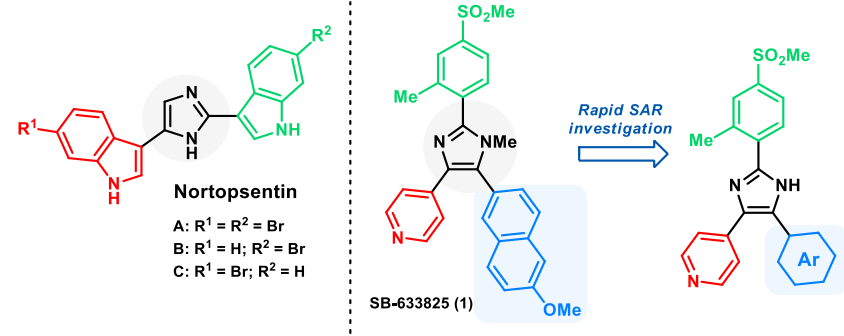

Figure 1. Bioactive Nortopsentins A-C and pyridyl-imidazole kinase inhibitor SB-633825 (1).

relationship (SAR) of trisubstituted pyridyl-imidazole $\mathbf{1}$ (Figure 1), focusing on changes in the naphthyl moiety since the previous synthetic approach introduced the naphthalene in the first step of a six-step route. ${ }^{20}$

Previous work from Laufer ${ }^{12}$ and Springer ${ }^{21}$ already provided access to trisubstituted pyridyl imidazoles in a divergent and modular fashion, although with the use of protecting groups, thus increasing the step count by two (Figure 2). The use of oxalyl boronates by Yudin offers a regioselective, protecting group-free and modular approach to imidazoles. However, the key intermediate is accessed in five steps, and the cross-coupled product is obtained in moderate yields (Figure 2). ${ }^{11}$

Received: July 8, 2019

Published: August 28, 2019 


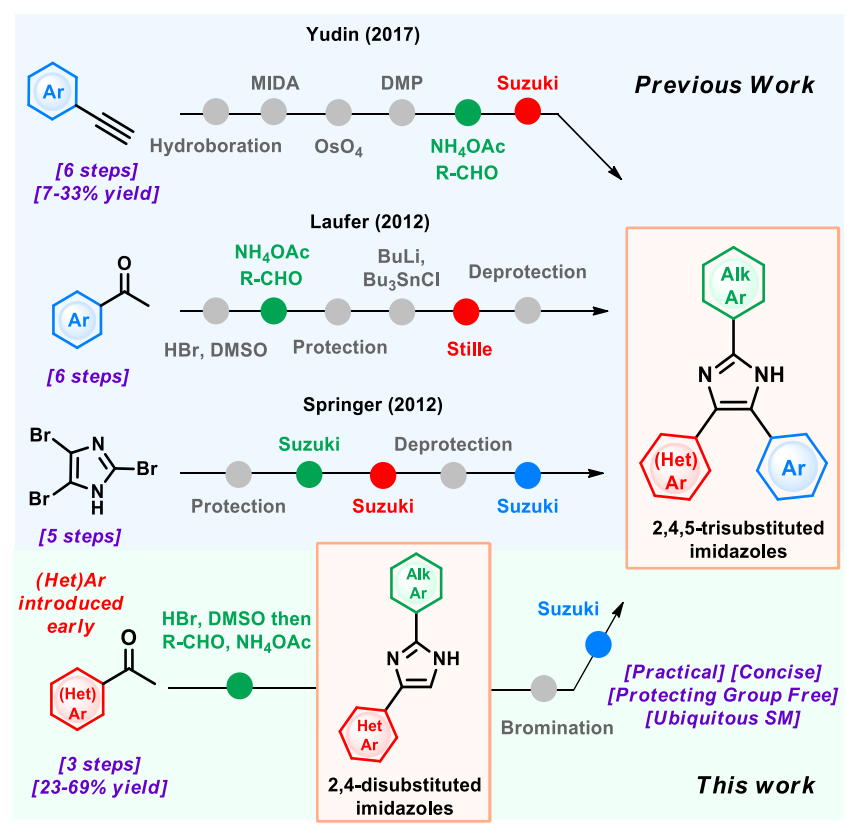

Figure 2. Modular access to 2,4,5-trisubstituted imidazoles.

We sought to address both challenges by implementing an efficient step- and redox-economical approach to disubstituted 2,4(5)-NH imidazoles, followed by the Suzuki reaction to introduce the aromatic substituent in a protecting group-free fashion. Herein, we report an improved one-pot approach to disubstituted 2,4(5)-NH imidazoles consisting of a sequential Kornblum oxidation, ${ }^{22}$ followed by Radziszewski ${ }^{23}$ imidazole condensation, which allowed the synthesis of twenty-nine $2,4(5)$-disubstituted imidazoles in yields ranging from $23 \%$ to $85 \%$. Moreover, representative imidazoles 32 and 36 were further functionalized to rapidly access a small kinase inhibitor library of 20 trisubstituted 2,4,5-NH imidazoles in yields ranging from $23 \%$ to $69 \%$ for three steps.

Initially, we investigated the possibility of obtaining the 2,4(5)-NH imidazole 5 employing the sequential oxidationcondensation protocol with acetophenone (2) and $p$ tolualdehyde (4) as representative carbonyl substrates. After extensive optimization, it was found that formation of glyoxal 3 from acetophenone (2) could be achieved, employing a catalytic amount $(10 \mathrm{~mol} \%)$ of aqueous $\mathrm{HBr}$ in DMSO at 85 ${ }^{\circ} \mathrm{C}$. After addition of a MeOH/DMSO $(6: 4 \mathrm{v} / \mathrm{v})$ solution of glyoxal to a mixture of $p$-tolualdehyde (4) and $\mathrm{NH}_{4} \mathrm{OAc}$ in $\mathrm{MeOH}$, the desired imidazole was isolated in $69 \%$ yield (Table 1 , entry 1).

Decreasing $\mathrm{HBr}$ loading (entries 2-5) resulted in longer reaction times (oxidation step) with some improvement in the yield. Importantly, an increase in reaction temperature did not have an impact on the yield (entries 4 and 5), but the oxidation reaction proceeded faster. When the reaction was carried out in the absence of $\mathrm{HBr}$ (entry 6), neither glyoxal 3 nor imidazole 5 were observed. When the amount of acetophenone was increased (entry 1), a better yield was observed and 1.25 equiv was selected as the optimum amount. Changing from $\mathrm{MeOH}$ to $\mathrm{EtOH}$ (oxidation step, entry 8) or adding polar aprotic (DMF and DMSO, entries 7 and 9) and apolar (PhMe, entry 10) solvent in the condensation step did not provide better yields. (See Table S1 for all conditions employed.)

The substrate scope (Scheme 1) was then explored using different methyl ketones and aldehydes. The transformation
Table 1. Optimization of the Reaction Conditions for the Synthesis of Disubstituted Imidazole 5

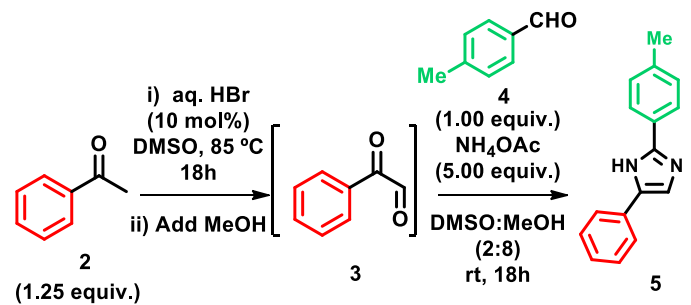

$\begin{array}{ccc}\text { Entry } & \text { Changes from the conditions described above } & \text { Yield }^{b} \\ 1 & \text { none } & 69(69) \\ 2 & 2 \text { (1.00 equiv), aq } \mathrm{HBr}(200 \mathrm{~mol} \%), 60{ }^{\circ} \mathrm{C}, 24 \mathrm{~h} & 48 \\ 3 & 2\left(1.00 \text { equiv), aq } \mathrm{HBr}(50 \mathrm{~mol} \%), 60^{\circ} \mathrm{C}, 72 \mathrm{~h}\right. & 57 \\ 4 & 2\left(1.00 \text { equiv), aq } \mathrm{HBr}(50 \mathrm{~mol} \%), 85^{\circ} \mathrm{C}, 12 \mathrm{~h}\right. & 55 \\ 5 & 2\left(1.00 \text { equiv), aq } \mathrm{HBr}(10 \mathrm{~mol} \%), 85^{\circ} \mathrm{C}, 18 \mathrm{~h}\right. & 61 \\ 6 & \mathrm{no} \mathrm{aq} \mathrm{HBr} & 0 \\ 7 & \mathrm{DMSO} / \mathrm{MeOH}(7: 3)^{c} & 45 \\ 8 & \mathrm{DMSO} / \mathrm{EtOH}(2: 8)^{c} & 49 \\ 9 & \text { DMSO/MeOH/DMF }(2: 3: 5)^{c} & 45 \\ 10 & \text { DMSO/MeOH/PhMe }(2: 3: 5)^{c} & 47 \\ 11 & \text { with isolation of } 3(\text { stepwise procedure }) & (52)\end{array}$

${ }^{a}$ Oxidation step performed using acetophenone (2) (Table 1), aqueous $\mathrm{HBr}(48 \% \mathrm{w} / \mathrm{w}, 8.9 \mathrm{M})$ (Table 1), and DMSO (0.50 M). Condensation step performed by slow addition $(30 \mathrm{~min}$ ) of glyoxal 3 solution in DMSO/MeOH (4:6, v/v, $0.19 \mathrm{M}$ relative to acetophenone $2)$ to a mixture of tolualdehyde (4) $(0.3 \mathrm{mmol})$ and $\mathrm{NH}_{4} \mathrm{OAc}(1.5$ $\mathrm{mmol})$ in $\mathrm{MeOH}(1.5 \mathrm{~mL}, 0.2 \mathrm{M})$. Final solvent composition: DMSO/MeOH (8:2). ${ }^{b}$ Yield after workup as determined by ${ }^{1} \mathrm{H}$ NMR analysis of the crude reaction mixture with 1,3,5-trimethoxybenzene as the internal standard. Isolated yield given in (parenthesis). ${ }^{c}$ Final solvent composition

proved to tolerate well the electronic properties of the substituted acetophenones employed. Notably, substituted acetophenones bearing electron-donating $(6,14)$, electronwithdrawing $(10,11,12,15)$, electron-neutral (13), and pyridine $(\mathbf{8})$ were good substrates for this transformation, and products were isolated in yields ranging from 56 to $72 \%$ (Scheme 1a). However, the 3 -indole and 2 -furyl derivatives 7 and 9, respectively, performed poorly under standard conditions while 2-hydroxyacetophenone (37) did not show reactivity even when higher amounts of $\mathrm{HBr}(300 \mathrm{~mol} \%)$ were employed. For 3-acetylindole (38), it was necessary to increase the $\mathrm{HBr}$ loading to $300 \mathrm{~mol} \%$ at $85{ }^{\circ} \mathrm{C}$ to accomplish consumption of the starting material, but the imidazole product was not obtained under these conditions. Saturated ketones (40 and 41) were consumed under standard oxidation conditions without formation of the imidazole product (Scheme 1d). Interestingly, when 4-aminoacetophenone (42) was reacted with $110 \mathrm{~mol} \%$ of $\mathrm{HBr}$, the brominated side product 43 was obtained (Scheme 1e).

Considering the aldehyde scope, benzaldehydes bearing electron-withdrawing groups $(17,18,20,21,23,24,27)$, such as halides and nitro groups, performed better than those bearing electron-donating groups $(19,22)$ with the exception of the phenolic derivative 16, which was isolated in $85 \%$ yield. This behavior might be due to the electron distribution in the aromatic ring of the substituted benzaldehyde, which is more reactive when electron-withdrawing groups are present. Interestingly, saturated cyclic aldehydes such as cyclopropyl (31) and cyclohexyl (28) carboxyaldehydes were good substrates for this transformation (70\% and $83 \%$ yield, 
Scheme 1. Scope of the Oxidation-Condensation Approach to 2,4(5)-Disubstituted Imidazoles

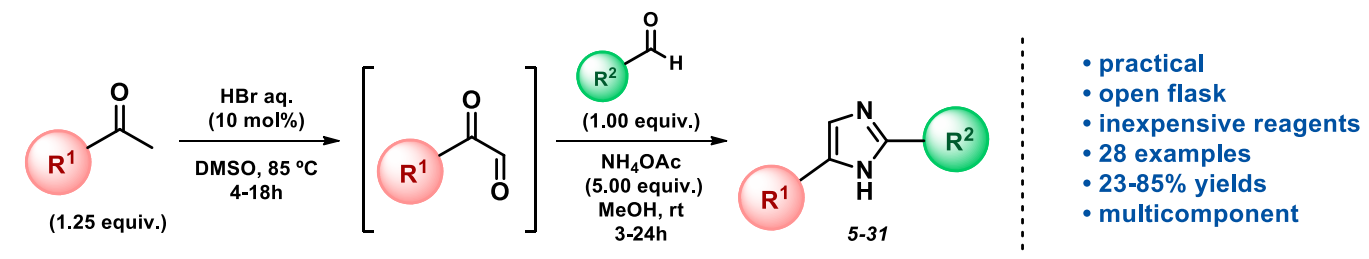

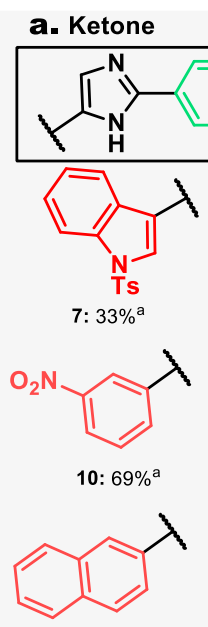

13: $67 \%^{\mathrm{a}}$

\section{(1)}

$3-24 h$

$5-31$

b. Aldehyde
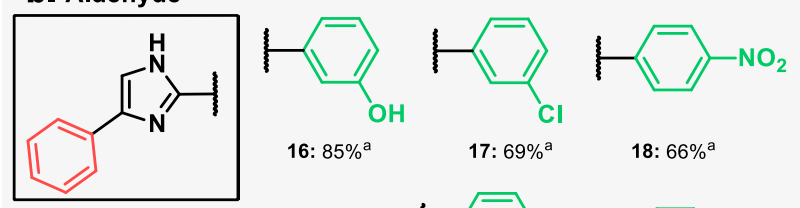

16: $85 \%^{\mathrm{a}}$

17: $69 \%^{\mathrm{a}}$

18: $66 \%^{\mathrm{a}}$
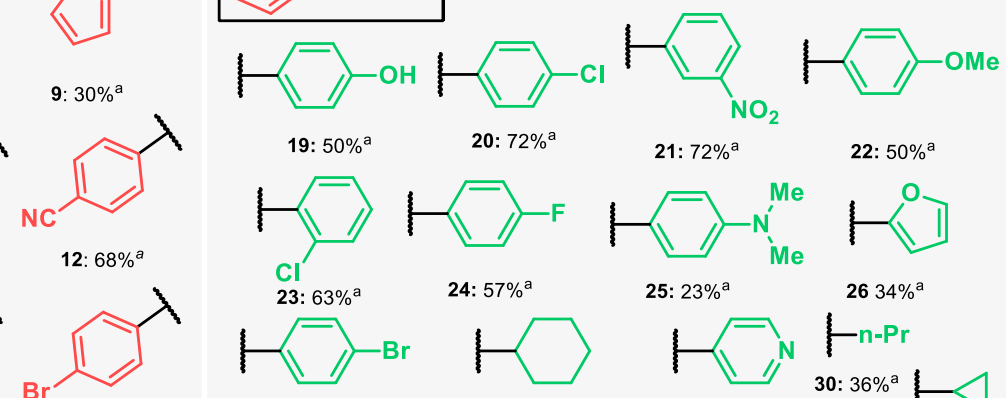

22: $50 \%^{a}$

12: $68 \%^{a}$
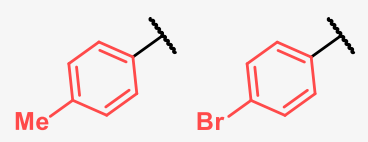

15: $72 \%^{a}$

27: $61 \%^{\mathrm{a}}$

28: $83 \%^{a}$

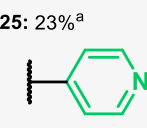

29: $40 \%^{\mathrm{a}}$

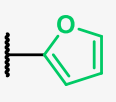

$2634 \%^{a}$

-n-Pr
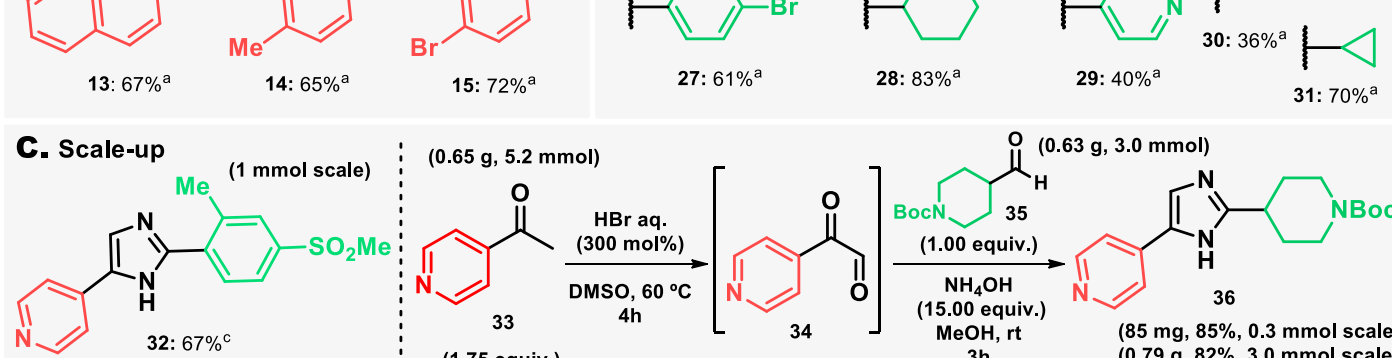

$\underbrace{\substack{\text { DMso, } 60^{\circ} \mathrm{C} \\ 43 \mathrm{~h}}}_{(1.75 \text { equiv. })}$

d. Incompatible ketones<smiles>CC(=O)c1ccccc1O</smiles>

37<smiles>CC(=O)c1c[nH]c2ccccc12</smiles>

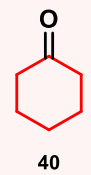

39

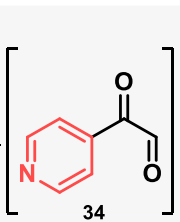

$\underset{\substack{(15.00 \text { equiv. }) \\ \text { MeOH, } r t}}{\stackrel{\mathrm{NH}_{4} \mathrm{OH}}{(1.00 \text { equiv. })}}$ $3 \mathrm{~h}$

31: $70 \%^{\mathrm{a}}$

${ }^{a}$ Reaction scale: $0.30 \mathrm{mmol}$. Reactions performed employing the ketone (1.25 equiv), aldehyde (1.00 equiv), $\mathrm{NH}_{4} \mathrm{OAc}(5.00$ equiv), DMSO (0.75 $\mathrm{mL}), \mathrm{MeOH}(2.75 \mathrm{~mL})$. Yields described correspond to isolated yields after column cromatography. Ketone (1.25 equiv), $48 \%$ aq $\mathrm{HBr}(10 \mathrm{~mol} \%)$, DMSO, $85^{\circ} \mathrm{C}, 18 \mathrm{~h}$ then aldehyde (1.00 equiv), $\mathrm{NH}_{4} \mathrm{OAc}$ ( 5.00 equiv), $\mathrm{MeOH}, \mathrm{rt}, 24 \mathrm{~h} .{ }^{b}$ Ketone ( 1.25 equiv), $48 \%$ aq $\mathrm{HBr}(300 \mathrm{~mol} \%), \mathrm{DMSO}$, $85^{\circ} \mathrm{C}, 8 \mathrm{~h}$ then aldehyde (1.00 equiv), $\mathrm{NH}_{4} \mathrm{OAc}$ ( 5.00 equiv), $\mathrm{MeOH}, \mathrm{rt}, 24 \mathrm{~h} .{ }^{c}$ Ketone (1.75 equiv), $48 \%$ aq $\mathrm{HBr}(300 \mathrm{~mol} \%), \mathrm{DMSO}, 85{ }^{\circ} \mathrm{C}, 18$ $\mathrm{h}$ then aldehyde (1.00 equiv), $\mathrm{NH}_{4} \mathrm{OAc}$ (10.00 equiv), $\mathrm{MeOH}, \mathrm{rt}, 24 \mathrm{~h}$.

respectively), although $n$-butyraldehyde derivative 30 was isolated in only $36 \%$. Overall, imidazoles 16-31 from the aldehyde scope were isolated in yields ranging from 23 to $85 \%$ from the corresponding aldehydes (Scheme 1b). The disubstituted imidazole 32 was obtained in $67 \%$ yield after optimization of the reaction conditions for this specific substrate. (See Table S2.) It was also possible to employ the commercially available Boc-protected aldehyde 35 under slightly modified conditions using $\mathrm{NH}_{4} \mathrm{OH}$ as a basic ammonia source to neutralize $\mathrm{HBr}$ in order to avoid unwanted deprotection. The disubstituted imidazole 36 was isolated in $85 \%$ yield in a $0.3 \mathrm{mmol}$ scale, and the reaction proved to be scalable in a $3.0 \mathrm{mmol}$ scale, affording 36 in $82 \%$ yield. (Scheme 1c). This one-pot approach for disubstituted imidazoles has the following advantages when compared to a stepwise procedure: (1) avoids glyoxal isolation, which can be troublesome; $^{23}$ (2) starts from ubiquitous and/or easily accessible starting materials; (3) employs aqueous $\mathrm{HBr}$ as the catalyst and DMSO as the oxidant, and (4) is amenable to scale-up. On the other hand, the 4(5)-position of the imidazole ring is restricted to aryl substitutents and is not compatible with acid-sensitive substrates, such as indoles. (Scheme $1 \mathrm{~d}$ ).

To show further applicability of the method, disubstituted imidazole 32 was functionalized at C-5 position of the imidazole ring to afford a small library of pyridyl imidazoles inhibitors for testing against STK10 and SLK kinases. ${ }^{15}$ This was accomplished by bromination of the 2,4-disubstituted imidazole $^{24}$ 32, followed by Suzuki-Miyaura cross-coupling ${ }^{25}$ with boronic acids or esters (Scheme $2 \mathrm{a}$ ). In this case, nineteen 2,4,5-trisubstituted imidazoles 46-64 were obtained in yields ranging from 37 to $97 \%$ from the common intermediate 44 (Scheme 2b). Interestingly, it was possible to perform a onepot Miyaura borylation and Suzuki cross-coupling starting from bromide 65 to access trisubstituted imidazole 67 in 52\% yield (Scheme 2c). Moreover, the trisubstituted imidazole 71, which was synthesized by Yudin in six steps ( $12 \%$ overall 
Scheme 2. Synthesis of 2,4,5-Trisubstituted Imidazole STK10 Kinase Inhibitors

a. Synthetic approach towards STK10 kinase inhibitors
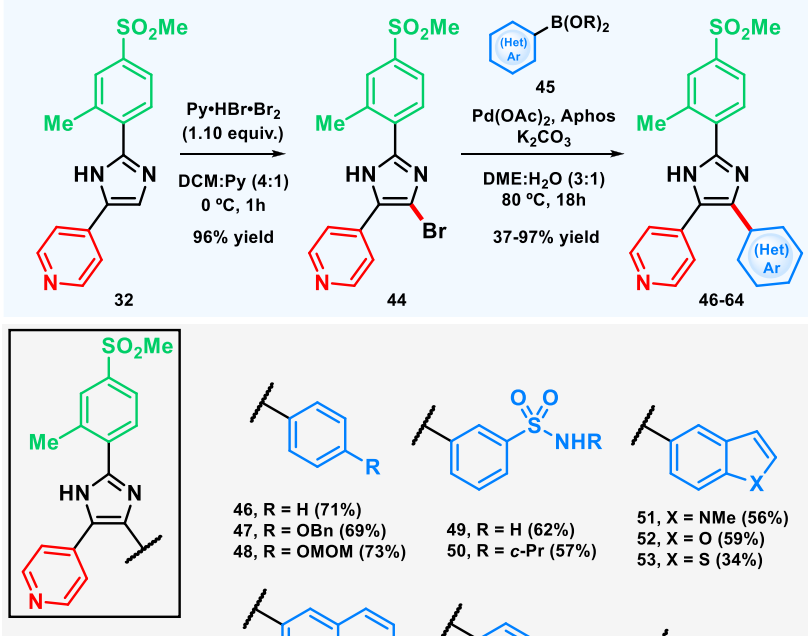

$46, R=H(71 \%)$
$47, R=O B n(69)$

$48, R=$ OMOM $(73 \%) \quad 50, R=c-\operatorname{Pr}(57 \%) \quad 52, X=O(59 \%)$
$53, X=S(34 \%)$
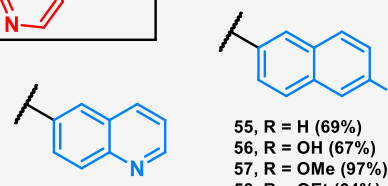

54: $52 \%$

$58, R=$ OEt $(64 \%)$

$59, \mathrm{R}=\mathrm{O} c-\operatorname{Pr}(63 \%)$

C. One-pot Miyaura Borylation-Suzuki Cross Coupling

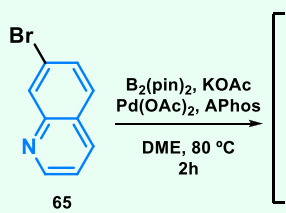

(.00

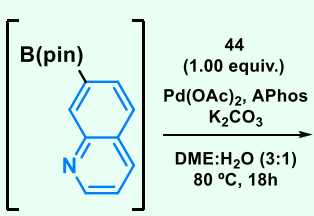

66
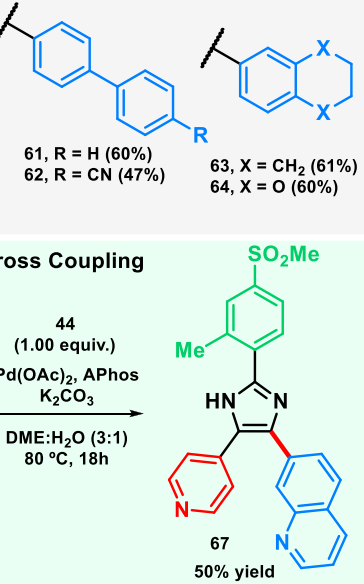

d. Efficient synthesis of imidazole 71 and access to GSK3037619A (72)

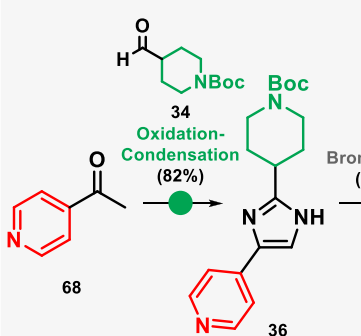

[Current] 3 steps $\left(69 \%, R^{1}=B o c\right)$ [Previous] 6 steps $\left(12 \%, R^{1}=\right.$ Boc $)$

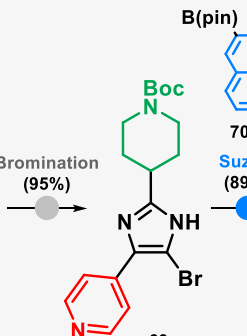

69

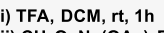
ii) $\mathrm{CH}_{2} \mathrm{O}, \mathrm{Na}(\mathrm{OAc})_{3} \mathrm{BH}$ $\mathrm{MeCN}, \mathrm{rt}, \mathbf{2 4 h}$ $80 \%$ yield

(pin)

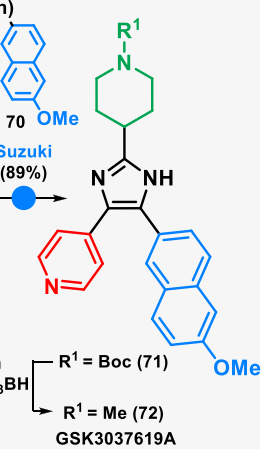

yield), ${ }^{11}$ could be accessed in three steps (69\% overall yield) from 4-acetylpyridine (68) employing the same strategy as for imidazoles 46-64 (Scheme 2d). From this advanced intermediate 71, the known inhibitor GSK3037619A (72) could be synthesized in a one-pot procedure in $80 \%$ yield (Scheme 2d).

Compounds $57(\mathrm{R}=\mathrm{OMe})$ and $59(\mathrm{R}=\mathrm{O} c-\mathrm{Pr})$ were subjected to binding displacement assays ${ }^{11}$ against STK10 and SLK kinases and displayed $K_{\mathrm{i}}$ values of 146 and $700 \mathrm{nM}$, respectively, against STK10, and 180 and $230 \mathrm{nM}$, respectively, for SLK. The weaker binding of the cyclopropyl derivative to STK10 might be explained by a more significant space restriction in the hydrophobic pocket of STK10 to bulkier substituents at the 6-position compared to SLK.

In conclusion, we developed an improved one-pot procedure for the synthesis of 2,4(5)-disubstituted $\mathrm{NH}$-imidazoles, employing widely available starting materials such as methyl ketones and aldehydes, and demonstrated the utility of the methodology by using it as a key step in a short, modular, and divergent synthetic route to 2,4,5-trisubstituted pyridylimidazole inhibitors of the STK10 kinase and for the synthesis of the GSK3037619A in 4 steps (55\% overall yield). This approach enabled rapid exploration of the SAR at the C-5 position of the imidazole ring and permits regioselective variation at the $\mathrm{C}-2$ and $\mathrm{C}-4$ positions for future exploration.

\section{EXPERIMENTAL SECTION}

General Information. Unless stated otherwise, the synthesis of 2,4-disubstituted imidazoles was performed using an undistilled solvent, without any precaution to exclude air and moisture, in 5 $\mathrm{mL}$ vials, and the mixture was stirred with Teflon-coated magnetic bars $(1 \mathrm{~cm} \times 0.5 \mathrm{~cm})$. Suzuki couplings for the preparation of $2,4,5$ trisubstituted imidazoles were performed under a nitrogen atmosphere in $100 \mathrm{~mm} \times 13 \mathrm{~mm}(9 \mathrm{~mL})$ culture tubes and were stirred with Teflon-coated magnetic bars $(1 \mathrm{~cm} \times 0.5 \mathrm{~cm})$. Dry dimethoxyethane (DME, 99.5\%) and dry dimethylformamide (DMF, 99.5\%) were purchased from Sigma-Aldrich and stored under $3 \AA$ molecular sieves and nitrogen-purged before use. Dichloromethane (DCM) and triethylamine $\left(\mathrm{Et}_{3} \mathrm{~N}\right)$ were pretreated with calcium hydride and distilled before use. Pyridine was distilled from calcium hydride and stored over $4 \AA$ molecular sieves. Tetrahydrofuran was dried over $4 \AA$ molecular sieves and distilled from sodium metal and benzophenone before use. All other solvents and commercial reagents were used as supplied without further purification unless stated otherwise. All reactions involving heating were carried out using aluminum blocks and a contact thermometer. Reactions were monitored by thin-layer chromatography (silica gel 60 F254 in aluminum foil, Merck), and visualization was achieved under UV light $(254 \mathrm{~nm})$ followed by staining in potassium permanganate $\left(\mathrm{KMnO}_{4}\right)$, Dragendorff stain (Dragendorff), dinitrophenylhydrazine stain (DNFH), $p$-anisaldehyde stain ( $p$-ASD), or curcumin stain and heating. Silica gel 60 F254 (200-400 Mesh, Merck) was used for purifications by standard flash column chromatography. NMR spectra were recorded on a Bruker Avance DPX $250 \mathrm{MHz}\left(250 \mathrm{MHz}{ }^{1} \mathrm{H}, 63 \mathrm{MHz}{ }^{13} \mathrm{C}\right)$, Bruker Avance III $400\left(400 \mathrm{MHz}{ }^{1} \mathrm{H}, 101 \mathrm{MHz}{ }^{13} \mathrm{C}\right)$, Bruker Avance III $500(500$ $\left.\mathrm{MHz}{ }^{1} \mathrm{H}, 126 \mathrm{MHz}{ }^{13} \mathrm{C}\right)$, or Bruker Avance III $600\left(600 \mathrm{MHz}{ }^{1} \mathrm{H}\right.$, $151 \mathrm{MHz}{ }^{13} \mathrm{C}$ ) unit. The chemical shifts are expressed in parts per million (ppm) relative to the residual solvent signal as an internal reference $\left([1] \mathrm{CDCl}_{3}{ }^{1} \mathrm{H}\right.$ RMN $=7.26,{ }^{13} \mathrm{C} \mathrm{RMN}=77.16$; [2] DMSO- $d_{6}{ }^{1} \mathrm{H}$ RMN $=2.50,{ }^{13} \mathrm{C} \mathrm{RMN}=39.52$; [3] acetone- $d_{6}:{ }^{1} \mathrm{H}$ $\mathrm{RMN}=2.05,{ }^{13} \mathrm{C} \mathrm{RMN}=206.26$; [4] methanol- $d_{4}:{ }^{1} \mathrm{H} \mathrm{RMN}=3.31$, ${ }^{13} \mathrm{C}$ RMN = 49.00). Multiplicities are reported with the following symbols: $\mathrm{s}=$ singlet, $\mathrm{d}=$ doublet, $\mathrm{t}=$ triplet, $\mathrm{q}=$ quartet, $\mathrm{m}=$ multiplet and multiples thereof. High-resolution mass spectra (ESI) were acquired on an Xevo Q-Tof mass spectrometer (Waters, Manchester, U.K.) equipped with a nanoESI type ionization source. IR spectra were recorded using a Thermo Scientific Nicolet IS5 spectrometer, using Thermo Scientific ID3 ATR. Melting points were recorded on a MP50 Metler-Toledo melting point apparatus and are uncorrected. STK10 and SLK binding displacement assays were performed as previously described. ${ }^{11}$

Optimization of the Reaction Conditions. 5-Phenyl-2-( $p$ tolyl)-1H-imidazole (5). A $6 \mathrm{~mL}$ vial was charged with acetophenone $2(46.0 \mathrm{mg}, 0.375 \mathrm{mmol}, 1.25$ equiv), DMSO $(0.75 \mathrm{~mL}, 0.5 \mathrm{M})$, concentrated aqueous $\mathrm{HBr}(48 \% \mathrm{w} / \mathrm{w}, 8.9 \mathrm{M})(4.24 \mu \mathrm{L}, 0.03 \mathrm{mmol}$, $10 \mathrm{~mol} \%)$, deionized water $(71 \mu \mathrm{L})$, and a magnetic stirr bar under air. The reaction mixture was stirred in a preheated aluminum block at $85{ }^{\circ} \mathrm{C}$ and was followed by TLC analysis $(30 \%$ EtOAc/hexane, $p$ ASD). After consumption of the starting material, the reaction mixture was cooled to room temperature and diluted with $\mathrm{MeOH}$ 
(1.25 mL, 0.19 M, final concentration relative to acetophenone (2), 2:8 mixture of $\mathrm{DMSO} / \mathrm{MeOH}$ ). This stock $\mathrm{DMSO} / \mathrm{MeOH}$ solution was added dropwise over $30 \mathrm{~min}$ via syringe to a $6 \mathrm{~mL}$ vial containing $p$-tolualdehyde (4) $\left(37.0 \mathrm{mg}, 0.30 \mathrm{mmol}, 1.00\right.$ equiv), $\mathrm{NH}_{4} \mathrm{OAc}(116$ $\mathrm{mg}, 1.50 \mathrm{mmol}, 5.00$ equiv), and $\mathrm{MeOH}(1.5 \mathrm{~mL}, 0.2 \mathrm{M}$ in relation to 4). The reaction mixture was stirred at room temperature for $18 \mathrm{~h}$ and then poured directly into a separatory funnel containing a mixture of saturated $\mathrm{NaHCO}_{3}$ and saturated $\mathrm{Na}_{2} \mathrm{~S}_{2} \mathrm{O}_{3}(1: 1,1 \times 20 \mathrm{~mL})$ and EtOAc $(10 \mathrm{~mL})$. The phases were separated, and the aqueous phase was extracted with EtOAc $(5 \times 5 \mathrm{~mL})$. The organic phases were combined, washed with saturated $\mathrm{NaCl}$ solution $(1 \times 5 \mathrm{~mL})$, dried over $\mathrm{Na}_{2} \mathrm{SO}_{4}$, filtered, and concentrated in the rotaevaporator. The residue was diluted with $\mathrm{EtOAc}(5 \mathrm{~mL})$, and a $1 \mathrm{~mL}$ aliquot was taken and concentrated in vacuo. To this were added 1,3,5-trimethoxybenzene $(10.2 \mathrm{mg}, 0.06 \mathrm{mmol})$ and acetone- $d_{6}(0.6 \mathrm{~mL})$, and the sample was analyzed by ${ }^{1} \mathrm{H}$ NMR. The crude mixtures were combined and purification of the residue by silica gel chromatography, eluting with EtOAc in hexanes $(19 \mathrm{~cm} \times 20 \mathrm{~mm}$, gradient elution, $0 \% \rightarrow$ $30 \%, 5 \%$ increases, $50 \mathrm{~mL}$ runs, $5-10 \mathrm{~mL}$ fractions), yielded 5 as a white solid ( $69 \%$ yield, $48.0 \mathrm{mg}$, $0.21 \mathrm{mmol}): R_{f}=0.30$ (30\% EtOAc/ hexane, UV, Dragendorff stain); ${ }^{1} \mathrm{H}$ NMR (500 MHz, DMSO- $\left.d_{6}\right) \delta$ $12.55(\mathrm{~s}, 1 \mathrm{H}), 7.89$ (d, $J=7.9 \mathrm{~Hz}, 2 \mathrm{H}), 7.86(\mathrm{~d}, J=7.6 \mathrm{~Hz}, 2 \mathrm{H}), 7.72$ $(\mathrm{s}, 1 \mathrm{H}), 7.36(\mathrm{t}, J=7.5 \mathrm{~Hz}, 2 \mathrm{H}), 7.28(\mathrm{~d}, J=7.9 \mathrm{~Hz}, 2 \mathrm{H}), 7.20(\mathrm{t}, J=$ $7.2 \mathrm{~Hz}, 1 \mathrm{H}), 2.34(\mathrm{~s}, 3 \mathrm{H}) ;{ }^{13} \mathrm{C}\left\{{ }^{1} \mathrm{H}\right\}$ NMR $\left(126 \mathrm{MHz}, \mathrm{DMSO}-d_{6}\right) \delta$ $146.0,140.9,137.5,134.8,129.3,128.4,126.1,125.2$, 124.9, 124.4, $114.0,20.9$. Spectroscopic data are in accordance with the literature. ${ }^{26}$

Ketone Scope: General Procedure A. A $6 \mathrm{~mL}$ vial was charged with the corresponding acetophenone $(0.375 \mathrm{mmol}, 1.25$ equiv), DMSO $(0.75 \mathrm{~mL}, 0.5 \mathrm{M})$, concentrated aqueous $\mathrm{HBr}(48 \% \mathrm{w} / \mathrm{w}, 8.9$ M) $(4.24 \mu \mathrm{L}, 0.03 \mathrm{mmol}, 10 \mathrm{~mol} \%)$, deionized water $(71 \mu \mathrm{L})$, and a magnetic stir bar under air. The reaction mixture was stirred in a preheated aluminum block at $85{ }^{\circ} \mathrm{C}$ and was followed by TLC analysis (EtOAc/hexane, $p$-ASD). After consumption of the starting material, the reaction mixture was cooled to room temperature and diluted with $\mathrm{MeOH}(1.25 \mathrm{~mL}, 0.19 \mathrm{M}$, final concentration relative to the corresponding acetophenone, 4:6 mixture of $\mathrm{DMSO} / \mathrm{MeOH}$ ). This stock DMSO/MeOH solution was added dropwise over $30 \mathrm{~min}$ via syringe to a $6 \mathrm{~mL}$ vial containing benzaldehyde $(32.0 \mathrm{mg}, 0.30$ mmol, 1.00 equiv), $\mathrm{NH}_{4} \mathrm{OAc}(116 \mathrm{mg}, 1.50 \mathrm{mmol}, 5.00$ equiv), and $\mathrm{MeOH}$ ( $1.5 \mathrm{~mL}, 0.2 \mathrm{M}$ in relation to benzaldehyde). The reaction mixture was stirred at room temperature for $18 \mathrm{~h}$ and then poured directly into a separatory funnel containing a mixture of saturated $\mathrm{NaHCO}_{3}$ and saturated $\mathrm{Na}_{2} \mathrm{~S}_{2} \mathrm{O}_{3}(1: 1,1 \times 20 \mathrm{~mL})$ and EtOAc $(10$ $\mathrm{mL}$ ). The phases were separated, and the aqueous phase was extracted with EtOAc $(5 \times 5 \mathrm{~mL})$. The organic phases were combined, washed with saturated $\mathrm{NaCl}$ solution $(1 \times 5 \mathrm{~mL})$, dried over $\mathrm{Na}_{2} \mathrm{SO}_{4}$, filtered, and concentrated in the rotaevaporator. The residue was purified by silica gel column chromatography.

Aldehyde Scope: General Procedure B. A $6 \mathrm{~mL}$ vial was charged with acetophenone $(46.0 \mathrm{mg}, 0.375 \mathrm{mmol}, 1.25$ equiv), DMSO $(0.75 \mathrm{~mL}, 0.5 \mathrm{M})$, concentrated aqueous $\mathrm{HBr}(48 \% \mathrm{w} / \mathrm{w}, 8.9$ M) $(4.24 \mu \mathrm{L}, 0.0375 \mathrm{mmol}, 10 \mathrm{~mol} \%)$, deionized water $(71 \mu \mathrm{L})$, and a magnetic stir bar under air. The reaction mixture was stirred in a preheated aluminum block at $85{ }^{\circ} \mathrm{C}$ and was followed by TLC analysis (EtOAc/hexane, $p$-ASD). After consumption of the starting material, the reaction mixture was cooled to room temperature and diluted with $\mathrm{MeOH}(1.25 \mathrm{~mL}, 0.19 \mathrm{M}$, final concentration relative to the corresponding acetophenone, 4:6 mixture of $\mathrm{DMSO} / \mathrm{MeOH}$ ). This stock DMSO/MeOH solution was added dropwise over $30 \mathrm{~min}$ via syringe to a $6 \mathrm{~mL}$ vial containing the corresponding aldehyde ( $0.30 \mathrm{mmol}, 1.00$ equiv), $\mathrm{NH}_{4} \mathrm{OAc}(116 \mathrm{mg}, 1.50 \mathrm{mmol}, 5.00$ equiv), and $\mathrm{MeOH}(1.5 \mathrm{~mL}, 0.2 \mathrm{M}$ in relation to the aldehyde). The reaction mixture was stirred at room temperature for $18 \mathrm{~h}$ and then poured directly into a separatory funnel containing a mixture of saturated $\mathrm{NaHCO}_{3}$ and saturated $\mathrm{Na}_{2} \mathrm{~S}_{2} \mathrm{O}_{3}(1: 1,1 \times 20 \mathrm{~mL})$ and EtOAc $(10$ $\mathrm{mL})$. The phases were separated, and the aqueous phase was extracted with EtOAc $(5 \times 5 \mathrm{~mL})$. The organic phases were combined, washed with saturated $\mathrm{NaCl}$ solution $(1 \times 5 \mathrm{~mL})$, dried over $\mathrm{Na}_{2} \mathrm{SO}_{4}$, filtered, and concentrated in the rotaevaporator. The residue was purified by silica gel column chromatography.
4-(4-Methoxyphenyl)-2-phenyl-1H-imidazole (6). The title compound was prepared according to general procedure A, using 4 'methoxyacetophenone $(58.0 \mathrm{mg}, 0.375 \mathrm{mmol}, 1.25$ equiv) and benzaldehyde ( $32.0 \mathrm{mg}, 0.30 \mathrm{mmol}, 1.00$ equiv). Purification by silica gel chromatography, eluting with EtOAc in hexanes $(19 \mathrm{~cm} \times 20 \mathrm{~mm}$, gradient elution, $0 \% \rightarrow 30 \%, 5 \%$ increases, $50 \mathrm{~mL}$ runs, $5-10 \mathrm{~mL}$ fractions), yielded 6 as a pale yellow solid (60\% yield, $44.0 \mathrm{mg}, 0.18$ $\mathrm{mmol}): R_{f}=0.12$ (30\% EtOAc/hexane, Dragendorff stain); ${ }^{1} \mathrm{H}$ NMR $\left(500 \mathrm{MHz}, \mathrm{DMSO}-d_{6}\right) \delta 12.55(\mathrm{br} \mathrm{s}, 1 \mathrm{H}), 7.99(\mathrm{~d}, J=7.4 \mathrm{~Hz}, 2 \mathrm{H})$, $7.78(\mathrm{~d}, J=8.7 \mathrm{~Hz}, 2 \mathrm{H}), 7.63,(\mathrm{~d}, J=1.4 \mathrm{~Hz}, 1 \mathrm{H}), 7.46(\mathrm{t}, J=7.8 \mathrm{~Hz}$, $2 \mathrm{H}), 7.35(\mathrm{t}, J=7.3 \mathrm{~Hz}, 1 \mathrm{H}), 6.95(\mathrm{~d}, J=8.7 \mathrm{~Hz}, 2 \mathrm{H}), 3.77(\mathrm{~s}, 3 \mathrm{H})$; ${ }^{13} \mathrm{C}\left\{{ }^{1} \mathrm{H}\right\}$ NMR (126 MHz, DMSO-d $\left.d_{6}\right) \delta 158.3,157.9,146.3,145.5$, $141.0,130.7,128.7,128.6,128.0,127.5,125.9,125.6,125.5,125.0$, $124.8,114.3,113.9,113.0,55.2,55.0 ; \nu_{\max }\left(\mathrm{cm}^{-1}\right.$, thin film, ATR $)$ 2925 (br), $1602(\mathrm{~s}), 1517(\mathrm{w}), 1480(\mathrm{w}), 1443(\mathrm{w}), 1312(\mathrm{~s}), 1213$ (w), $1147(\mathrm{~s}), 1109(\mathrm{~m}), 1075(\mathrm{w}), 1075(\mathrm{w}), 999(\mathrm{w}), 958(\mathrm{~m}), 877$ (w), $768(\mathrm{~s}), 762(\mathrm{~s}), 744(\mathrm{~s}), 733(\mathrm{~s}), 700(\mathrm{~m})$; HRMS (ESI+/TOF) $m / z[\mathrm{M}+\mathrm{H}]^{+}$calcd for $\mathrm{C}_{16} \mathrm{H}_{15} \mathrm{~N}_{2} \mathrm{O} 251.1184$, found 251.1173; mp $175.0-177.8^{\circ} \mathrm{C}$ (EtOAc) (lit. $\left.170-174{ }^{\circ} \mathrm{C}\right)$. Spectroscopic data are in accordance with the literature. ${ }^{27}$

3-(2-Phenyl-1H-imidazol-5-yl)-1-tosyl-1H-indole (7). The title compound was prepared according to general procedure A, using 1(1-tosyl-1H-indol-3-yl)ethanone (S2) $(58.0 \mathrm{mg}, 0.375 \mathrm{mmol}, 1.25$ equiv) and benzaldehyde ( $32.0 \mathrm{mg}, 0.30 \mathrm{mmol}, 1.00$ equiv). Purification by silica gel chromatography, eluting with EtOAc in hexanes $(17 \mathrm{~cm} \times 20 \mathrm{~mm}$, gradient elution, $0 \% \rightarrow 60 \%, 5 \%$ increases, $45 \mathrm{~mL}$ runs, $15 \mathrm{~mL}$ fractions), yielded 7 as a white solid (33\% yield, $44.0 \mathrm{mg}, 0.11 \mathrm{mmol}): R_{f}=0.17(30 \% \mathrm{EtOAc} / \mathrm{hexane}, \mathrm{UV}$, Dragendorff stain); ${ }^{1} \mathrm{H}$ NMR (600 MHz, DMSO- $\left.d_{6} / \mathrm{D}_{2} \mathrm{O} / \mathrm{TFA}\right) \delta$ $8.54(\mathrm{~s}, 1 \mathrm{H}), 8.30(\mathrm{~s}, 1 \mathrm{H}), 8.10-8.07(\mathrm{~m}, 2 \mathrm{H}), 8.01(\mathrm{~d}, J=8.3 \mathrm{~Hz}$, $1 \mathrm{H}), 7.99(\mathrm{~d}, J=8.0 \mathrm{~Hz}, 1 \mathrm{H}), 7.91(\mathrm{~d}, J=8.5 \mathrm{~Hz}, 2 \mathrm{H}), 7.71-7.68$ $(\mathrm{m}, 3 \mathrm{H}), 7.49(\mathrm{t}, J=7.8 \mathrm{~Hz}, 1 \mathrm{H}), 7.44-7.39(\mathrm{~m}, 3 \mathrm{H}), 2.30(\mathrm{~s}, 3 \mathrm{H})$; ${ }^{13} \mathrm{C}\left\{{ }^{1} \mathrm{H}\right\}$ NMR (151 MHz, DMSO- $\left.d_{6} / \mathrm{D}_{2} \mathrm{O} / \mathrm{TFA}\right) \delta 146.6,144.5$, $134.5,133.8,132.8,130.8,129.9,127.5,127.2,127.0,126.4,126.3$, $125.6,124.8,123.1,121.0,117.3,113.8,109.8,21.3 ; \nu_{\max }\left(\mathrm{cm}^{-1}\right.$, thin film, ATR) 2847 (br), $1594(\mathrm{w}), 1460(\mathrm{w}), 1445(\mathrm{~m}), 1396(\mathrm{w})$, $1376(\mathrm{~m}), 1304(\mathrm{w}), 1279(\mathrm{w}), 1176(\mathrm{~s}), 1133(\mathrm{~m}), 1113(\mathrm{~m}), 1092$ (m), $1050(\mathrm{w}), 1024(\mathrm{w}), 985(\mathrm{~m}), 966(\mathrm{w}), 903(\mathrm{w}), 817(\mathrm{w}), 746$ (s), $709(\mathrm{~s}), 688(\mathrm{~s}), 660(\mathrm{~s})$; HRMS (ESI+/TOF) $\mathrm{m} / z[\mathrm{M}+\mathrm{H}]^{+}$ calcd for $\mathrm{C}_{24} \mathrm{H}_{20} \mathrm{~N}_{3} \mathrm{O}_{2} \mathrm{~S}$ 414.1276, found 414.1264; mp $249.0{ }^{\circ} \mathrm{C}$ (dec).

4-(2-Phenyl-1H-imidazol-5-yl)pyridine (8). The title compound was prepared according to general procedure $\mathrm{A}$, using 4-acetylpyridine (68) $(47.0 \mathrm{mg}, 0.375 \mathrm{mmol}, 1.25$ equiv) ,benzaldehyde $(32.0 \mathrm{mg}, 0.30$ mmol, 1.00 equiv) and $48 \%$ aq. $\mathrm{HBr}(300 \mathrm{~mol} \%)$. Purification by silica gel chromatography, eluting with $\mathrm{MeOH}$ in DCM $(19 \mathrm{~cm} \times 20$ $\mathrm{mm}$, gradient elution, $0 \% \rightarrow 6 \%, 0.5 \%$ increases, $30 \mathrm{~mL}$ runs, $7 \mathrm{~mL}$ fractions), yielded 8 as a yellow solid ( $56 \%$ yield, $37.0 \mathrm{mg}, 0.17$ mmol): $R_{f}=0.18$ (EtOAc, Dragendorff stain); ${ }^{1} \mathrm{H} \mathrm{NMR}(500 \mathrm{MHz}$, DMSO-d $\left.d_{6}\right) \delta 12.90($ br s, $1 \mathrm{H}), 8.53(\mathrm{~d}, J=5.0 \mathrm{~Hz}, 2 \mathrm{H}), 8.10-7.98$ $(\mathrm{m}, 3 \mathrm{H}), 7.80(\mathrm{~d}, J=6.0 \mathrm{~Hz}, 2 \mathrm{H}), 7.49(\mathrm{t}, J=7.4 \mathrm{~Hz}, 2 \mathrm{H}), 7.39(\mathrm{t}, J$ $=7.4 \mathrm{~Hz}, 2 \mathrm{H}) ;{ }^{13} \mathrm{C}\left\{{ }^{1} \mathrm{H}\right\}$ NMR $\left(125 \mathrm{MHz}\right.$, DMSO-d $d_{6} \delta 150.3,147.2$, $142.1,139.1,130.7,129.3,129.0,125.6,119.3,117.9 ; \nu_{\max }\left(\mathrm{cm}^{-1}\right.$, thin film, ATR) 2923, 1601, 1571, 1493, 1458, 1424, 1159, 1093, 999, 950, $821,838,780,774,712,705,694,685,677$; HRMS (ESI+/TOF) $\mathrm{m} /$ $z[\mathrm{M}+\mathrm{H}]^{+}$calcd for $\mathrm{C}_{14} \mathrm{H}_{12} \mathrm{~N}_{3}$ 222.1031, found 222.1037; mp 209.5-210.6 ${ }^{\circ} \mathrm{C}$ (lit. $212-214{ }^{\circ} \mathrm{C}$ ). Spectroscopic data are in accordance with the literature. ${ }^{10}$

4-(Furan-2-yl)-2-phenyl-1H-imidazole (9). The title compound was prepared according to general procedure $\mathrm{A}$, using 4-acetylfuran (41.0 mg, $0.375 \mathrm{mmol}, 1.25$ equiv) and benzaldehyde ( $32.0 \mathrm{mg}, 0.30$ mmol, 1.00 equiv). Purification by silica gel chromatography, eluting with EtOAc in hexanes $(18 \mathrm{~cm} \times 15 \mathrm{~mm}$, gradient elution, $0 \% \rightarrow$ $35 \%, 5 \%$ increases, $30 \mathrm{~mL}$ runs, $7 \mathrm{~mL}$ fractions), yielded a yellow oil, which was triturated with $5 \% \mathrm{DCM} /$ hexanes to yield 9 as a white solid (30\% yield, $19.0 \mathrm{mg}, 0.09 \mathrm{mmol}): R_{f}=0.33$ (30\% EtOAc/ hexane, Dragendorff stain); ${ }^{1} \mathrm{H}$ NMR $\left(500 \mathrm{MHz}\right.$, DMSO- $\left.d_{6}\right) \delta 12.71$ (br s, $1 \mathrm{H}), 7.97(\mathrm{~d}, J=7.3,2 \mathrm{H}), 7.62(\mathrm{~s}, 1 \mathrm{H}), 7.52(\mathrm{~s}, 1 \mathrm{H}), 7.46(\mathrm{t}, J$ $=7.6 \mathrm{~Hz}, 2 \mathrm{H}), 7.37(\mathrm{t}, J=7.4 \mathrm{~Hz}, 1 \mathrm{H}) 6.59(\mathrm{~s}, 1 \mathrm{H}), 6.53(\mathrm{~s}, 1 \mathrm{H})$; ${ }^{13} \mathrm{C}\left\{{ }^{1} \mathrm{H}\right\}$ NMR (126 MHz, DMSO- $\left.d_{6}\right) \delta 150.3,146.0,141.1,133.9$, 
$130.3,128.7,128.3,125.0,113.9,111.4,103.7 ; \nu_{\max }\left(\mathrm{cm}^{-1}\right.$, thin film, ATR) $3130(w), 2739(w, b r), 1560(w), 1494(w), 1460(w), 1407$ $(\mathrm{w}), 1297(\mathrm{w}), 1212(\mathrm{w}), 1160(\mathrm{~m}), 1143(\mathrm{~m}), 1092(\mathrm{w}), 1068(\mathrm{w})$, $1011(\mathrm{~m}), 969(\mathrm{~m}), 889(\mathrm{~m}), 786(\mathrm{~s}), 741(\mathrm{~s}), 719(\mathrm{~s}), 695(\mathrm{~s}), 681$ (s); HRMS (ESI+/TOF) $m / z[\mathrm{M}+\mathrm{H}]^{+}$calcd for $\mathrm{C}_{13} \mathrm{H}_{11} \mathrm{~N}_{2} \mathrm{O}$ 211.0871, found 211.0878; mp 145.4-148.7 ${ }^{\circ} \mathrm{C}$ (EtOAc) (lit. 154$\left.156{ }^{\circ} \mathrm{C}(\mathrm{EtOH})\right)$.

5-(3-Nitrophenyl)-2-phenyl-1H-imidazole (10). The title compound was prepared according to general procedure A, using $3^{\prime}$ nitroacetophenone $(63.0 \mathrm{mg}, 0.375 \mathrm{mmol}, 1.25$ equiv) and benzaldehyde ( $32.0 \mathrm{mg}, 0.30 \mathrm{mmol}, 1.00$ equiv). Purification by silica gel chromatography, eluting with EtOAc in hexanes $(18 \mathrm{~cm} \times$ $15 \mathrm{~mm}$, gradient elution, $0 \% \rightarrow 35 \%, 5 \%$ increases, $30 \mathrm{~mL}$ runs, $7 \mathrm{~mL}$ fractions), yielded 10 as a bright yellow solid (69\% yield, $55.0 \mathrm{mg}$, $0.21 \mathrm{mmol}): R_{f}=0.17$ (30\% EtOAc/hexane, Dragendorff stain); ${ }^{1} \mathrm{H}$ NMR $\left(500 \mathrm{MHz}\right.$, DMSO- $\left.d_{6}\right) \delta 12.87($ br s, $1 \mathrm{H}), 8.68(\mathrm{~s}, 1 \mathrm{H}), 8.30$ $(\mathrm{d}, J=7.7 \mathrm{~Hz}, 1 \mathrm{H}), 8.10-8.00(\mathrm{~m}, 4 \mathrm{H}) 7.67(\mathrm{t}, J=8.0 \mathrm{~Hz}, 1 \mathrm{H}), 7.49$ $(\mathrm{t}, J=7.6 \mathrm{~Hz}, 2 \mathrm{H}), 7.40(\mathrm{t}, J=7.2 \mathrm{~Hz}, 1 \mathrm{H}) ;{ }^{13} \mathrm{C}\left\{{ }^{1} \mathrm{H}\right\}$ NMR $(126$ MHz, DMSO- $\left.d_{6}\right) \delta 148.4,146.5,138.9,136.5,130.5,130.2,130.0$, 128.8, 128.4, 125.1, 120.7, 118.4, 116.3; $\nu_{\max }\left(\mathrm{cm}^{-1}\right.$, thin film, ATR $3383(\mathrm{~m}), 1561(\mathrm{w}), 1541(\mathrm{w}), 1516(\mathrm{~s}), 1290(\mathrm{w}), 1118(\mathrm{~m}), 1103$ (w), $893(\mathrm{~m}), 872(\mathrm{w}), 821(\mathrm{~m}), 782(\mathrm{~s}), 745(\mathrm{~s}), 737(\mathrm{~s}), 718(\mathrm{~s})$, $695(\mathrm{~s}), 687(\mathrm{~s})$; HRMS (ESI+/TOF) $\mathrm{m} / \mathrm{z}[\mathrm{M}+\mathrm{H}]^{+}$calcd for $\mathrm{C}_{15} \mathrm{H}_{12} \mathrm{~N}_{3} \mathrm{O}_{2}$ 266.0930, found 266.0933; mp 183.7-185.3 ${ }^{\circ} \mathrm{C}$ (EtOAc) (lit. $\left.181.1-183.9^{\circ} \mathrm{C}\right)$. Spectroscopic data are in accordance with the literature. ${ }^{28}$

5-(4-Nitrophenyl)-2-phenyl-1H-imidazole (11). The title compound was prepared according to general procedure A, using $4^{\prime}$ nitroacetophenone (63.0 mg, $0.375 \mathrm{mmol}, 1.25$ equiv) and benzaldehyde ( $32.0 \mathrm{mg}, 0.30 \mathrm{mmol}, 1.00$ equiv). Purification by silica gel chromatography, eluting with EtOAc in hexanes $(18 \mathrm{~cm} \times$ $15 \mathrm{~mm}$, gradient elution, $0 \% \rightarrow 35 \%, 5 \%$ increases, $30 \mathrm{~mL}$ runs, $7 \mathrm{~mL}$ fractions), yielded 11 as a bright yellow solid (64\% yield, $51.0 \mathrm{mg}$, $0.19 \mathrm{mmol}$ ): $R_{f}=0.20$ (30\% EtOAc/hexane, Dragendorff stain); ${ }^{1} \mathrm{H}$ NMR $\left(500 \mathrm{MHz}\right.$, DMSO- $\left.d_{6}\right) \delta 12.97($ br s, $1 \mathrm{H}), 8.25(\mathrm{~d}, J=8.8 \mathrm{~Hz}$, $2 \mathrm{H}), 8.14-8.10(\mathrm{~m}, 3 \mathrm{H}), 8.30(\mathrm{~d}, J=7.8 \mathrm{~Hz}, 2 \mathrm{H}), 7.49(\mathrm{t}, J=7.6 \mathrm{~Hz}$, $2 \mathrm{H}), 7.40(\mathrm{t}, J=7.4 \mathrm{~Hz}, 2 \mathrm{H}) ;{ }^{13} \mathrm{C}\left\{{ }^{1} \mathrm{H}\right\}$ NMR $\left(125 \mathrm{MHz}\right.$, DMSO- $\left.d_{6}\right)$ $\delta$ 147.0, 145.3, 141.4, 139.1, 130.1, 128.8, 125.1, 124.8, 124.1, 118.0; $\nu_{\max }\left(\mathrm{cm}^{-1}\right.$, ATR $): 3352,2359,2344,1598,1506,1489,1458,1333$, $1178,1131,1109,945,858,791,780,753,717,696$; HRMS (ESI +/TOF) $m / z[\mathrm{M}+\mathrm{H}]^{+}$calcd for $\mathrm{C}_{15} \mathrm{H}_{12} \mathrm{~N}_{3} \mathrm{O}_{2}$ 266.0930, found 266.0929; mp 187.2-188.7 ${ }^{\circ} \mathrm{C}$ (EtOAc) (lit. $190-191{ }^{\circ} \mathrm{C}$ ), turned brown upon heating. Spectroscopic data are in accordance with the literature. $^{29}$

4-(2-Phenyl-1H-imidazol-5-yl)benzonitrile (12). The title compound was prepared according to general procedure A, using 4acetylbenzonitrile (55.0 mg, $0.375 \mathrm{mmol}, 1.25$ equiv) and benzaldehyde $(32.0 \mathrm{mg}, 0.30 \mathrm{mmol}, 1.00$ equiv). Purification by silica gel chromatography, eluting with EtOAc in hexanes $(18 \mathrm{~cm} \times$ $15 \mathrm{~mm}$, gradient elution, $0 \% \rightarrow 60 \%, 5 \%$ increases, $12 \times 30 \mathrm{~mL}$ runs, then $10 \%$ increases, $2 \times 30 \mathrm{~mL}$ runs, $10 \mathrm{~mL}$ fractions), yielded 12 as a yellow solid $(68 \%$ yield, $50.0 \mathrm{mg}, 0.20 \mathrm{mmol}): R_{f}=0.18(30 \%$ EtOAc/hexane, Dragendorff stain); ${ }^{1} \mathrm{H}$ NMR (500 MHz, DMSO- $\left.d_{6}\right)$ $\delta 12.88($ br s, $1 \mathrm{H}), 8.10-7.97(\mathrm{~m}, 5 \mathrm{H}), 7.82(\mathrm{~d}, J=8.2 \mathrm{~Hz}, 2 \mathrm{H}), 7.49$ $(\mathrm{t}, J=7.7 \mathrm{~Hz}, 2 \mathrm{H}), 7.39(\mathrm{t}, J=7.5 \mathrm{~Hz}, 1 \mathrm{H}) ;{ }^{13} \mathrm{C}\left\{{ }^{1} \mathrm{H}\right\}$ NMR $(126$ $\left.\mathrm{MHz}, \mathrm{DMSO}-d_{6}\right) \delta 146.6,139.4,139.2,132.5,130.2,128.8,125.0$, $124.8,119.3,117.1,108.0 ; \nu_{\max }\left(\mathrm{cm}^{-1}\right.$, thin film, ATR) $3294(\mathrm{~m})$, $2923(\mathrm{w}), 2851(\mathrm{w}), 2539(\mathrm{w}), 2226(\mathrm{~m}), 1604(\mathrm{~m}), 1539(\mathrm{w}), 1491$ (w), $1458(\mathrm{w}), 1416(\mathrm{w}), 1133(\mathrm{~m}), 945(\mathrm{w}), 849(\mathrm{~m}), 728(\mathrm{~s}), 699$ (s); HRMS (ESI+/TOF) $m / z[\mathrm{M}+\mathrm{H}]^{+}$calcd for $\mathrm{C}_{16} \mathrm{H}_{12} \mathrm{~N}_{3}$ 246.1031, found 246.1032; mp 209.0-211.8 ${ }^{\circ} \mathrm{C}$ (EtOAc)

5-(Naphthalen-2-yl)-2-phenyl-1H-imidazole (13). The title compound was prepared according to general procedure $\mathrm{A}$, using $2^{\prime}$ acetonaphtone $(64.0 \mathrm{mg}, 0.375 \mathrm{mmol}, 1.25$ equiv) and benzaldehyde (32.0 mg, $0.30 \mathrm{mmol}, 1.00$ equiv). Purification by silica gel chromatography, eluting with $\mathrm{MeOH}$ in $\mathrm{DCM}(18 \mathrm{~cm} \times 15 \mathrm{~mm}$, gradient elution, $0 \% \rightarrow 5 \%, 0.5 \%$ increases, $30 \mathrm{~mL}$ runs, $7 \mathrm{~mL}$ fractions), yielded 13 as a pale yellow solid (67\% yield, $54.0 \mathrm{mg}, 0.20$ $\mathrm{mmol}): R_{f}=0.33$ (30\% EtOAc/hexane, Dragendorff stain); ${ }^{1} \mathrm{H}$ NMR $\left(500 \mathrm{MHz}, \mathrm{DMSO}-d_{6}\right) \delta 12.73(\mathrm{br} \mathrm{s}, 1 \mathrm{H}), 8.38(\mathrm{~s}, 1 \mathrm{H}), 8.10-8.00$ (m, 3H), 7.97-7.85 (m, 4H), 7.54-7.48 (m, 3H), $7.45(\mathrm{t}, J=7.5 \mathrm{~Hz}$, $1 \mathrm{H}), 7.38(\mathrm{t}, J=7.4 \mathrm{~Hz}, 1 \mathrm{H}) ;{ }^{13} \mathrm{C}\left\{{ }^{1} \mathrm{H}\right\}$ NMR $\left(126 \mathrm{MHz}\right.$, DMSO- $\left.d_{6}\right)$ $\delta 146.1,141.0,133.4,132.2,131.9,130.5,128.7,128.2,127.9,129.7$, $127.6,126.3,125.2,125.0,123.7,121.8,115.0 ; \nu_{\max }\left(\mathrm{cm}^{-1}\right.$, thin film, ATR) $2850,1630,1602,1572,1500,1484,1464,1454,1401,1263$, $1138,1126,1070,891,859,820,792,784,748,693$; HRMS (ESI +/TOF) $m / z[\mathrm{M}+\mathrm{H}]^{+}$calcd for $\mathrm{C}_{19} \mathrm{H}_{15} \mathrm{~N}_{2}$ 271.1235, found 271.1231; mp 223.9-225.0 ${ }^{\circ} \mathrm{C}(\mathrm{MeOH} / \mathrm{DCM})$.

5-(4-Methylphenyl)-2-phenyl-1H-imidazole (14). The title compound was prepared according to general procedure A, using $4^{\prime}$ methylacetophenone $(53.0 \mathrm{mg}, 0.375 \mathrm{mmol}, 1.25$ equiv) and benzaldehyde ( $32.0 \mathrm{mg}, 0.30 \mathrm{mmol}, 1.00$ equiv). Purification by silica gel chromatography, eluting with EtOAc in hexanes $(18 \mathrm{~cm} \times$ $15 \mathrm{~mm}$, gradient elution, $0 \% \rightarrow 25 \%, 5 \%$ increases, $30 \mathrm{~mL}$ runs, $7 \mathrm{~mL}$ fractions), yielded 14 as a pale yellow solid (65\% yield, $46.0 \mathrm{mg}, 0.20$ mmol): $R_{f}=0.37$ (30\% EtOAc/hexane, Dragendorff stain); ${ }^{1} \mathrm{H}$ NMR $\left(500 \mathrm{MHz}, \mathrm{DMSO}-d_{6}\right) \delta 12.60(\mathrm{br} \mathrm{s}, 1 \mathrm{H}), 8.00(\mathrm{~d}, J=7.1 \mathrm{~Hz}, 2 \mathrm{H})$, $7.80-7.64(\mathrm{~m}, 3 \mathrm{H}), 7.46(\mathrm{t}, J=7.8 \mathrm{~Hz}, 2 \mathrm{H}), 7.35(\mathrm{t}, J=7.3 \mathrm{~Hz}, 1 \mathrm{H})$, $7.18(\mathrm{~d}, J=7.0 \mathrm{~Hz}, 2 \mathrm{H}), 2.31(\mathrm{~s}, 3 \mathrm{H}) ;{ }^{13} \mathrm{C}\left\{{ }^{1} \mathrm{H}\right\}$ NMR $(126 \mathrm{MHz}$, DMSO- $\left.d_{6}\right) \delta 145.6,141.2,135.2,131.9,130.7,129.4,129.0,128.7$, $128.0,124.9,124.4,113.7,20.8 ; \nu_{\max }\left(\mathrm{cm}^{-1}\right.$, thin film, ATR $) 2985$, 1606, 1576, 1498, 1458, 1399, 1137, 1084, 962, 823, 803, 786, 721, 710, 695; HRMS (ESI+/TOF) $\mathrm{m} / z[\mathrm{M}+\mathrm{H}]^{+}$calcd for $\mathrm{C}_{16} \mathrm{H}_{15} \mathrm{~N}_{2}$ 235.1235, found 235.1222; mp 172.8-174.0 ${ }^{\circ} \mathrm{C}$ (EtOAc) (lit $179^{\circ} \mathrm{C}$ [benzene]), turned violet upon heating. Spectroscopic data are in accordance with the literature ${ }^{30,31}$

5-(4-Bromophenyl)-2-phenyl-1H-imidazole (15). The title compound was prepared according to general procedure A, using $4^{\prime}$ bromoacetophenone $(75.0 \mathrm{mg}, 0.375 \mathrm{mmol}, 1.25$ equiv) and benzaldehyde ( $32.0 \mathrm{mg}, 0.30 \mathrm{mmol}, 1.00$ equiv). Purification by silica gel chromatography, eluting with EtOAc in hexanes $(18 \mathrm{~cm} \times$ $15 \mathrm{~mm}$, gradient elution, $0 \% \rightarrow 25 \%, 5 \%$ increases, $30 \mathrm{~mL}$ runs, $7 \mathrm{~mL}$ fractions), yielded 15 as a pale yellow solid ( $72 \%$ yield, $65.0 \mathrm{mg}, 0.22$ mmol): $R_{f}=0.21$ (30\% EtOAc/hexane, Dragendorff stain); ${ }^{1} \mathrm{H}$ NMR $\left(500 \mathrm{MHz}, \mathrm{DMSO}-d_{6}\right) \delta 12.72(\mathrm{br} \mathrm{s}, 1 \mathrm{H}), 7.99(\mathrm{~d}, J=7.5 \mathrm{~Hz}, 2 \mathrm{H})$, 7.86-7.80 (m, 3H), $7.55(\mathrm{~d}, J=8.3 \mathrm{~Hz}, 2 \mathrm{H}), 7.47(\mathrm{t}, J=7.6 \mathrm{~Hz}$, $2 \mathrm{H}), 7.37(\mathrm{t}, J=7.4 \mathrm{~Hz}, 1 \mathrm{H}) ;{ }^{13} \mathrm{C}\left\{{ }^{1} \mathrm{H}\right\}$ NMR $\left(125 \mathrm{MHz}\right.$, DMSO- $\left.d_{6}\right)$ $\delta$ 146.1, 139.9, 134.0, 131.4, 130.4, 128.8, 128.3, 126.4, 125.0, 118.9, 115.0; $\nu_{\max }\left(\mathrm{cm}^{-1}\right.$, thin film, ATR) $2925(\mathrm{br}), 2360(\mathrm{w}), 1602(\mathrm{~s})$, $1517(\mathrm{w}), 1480(\mathrm{w}), 1443(\mathrm{w}), 1312(\mathrm{~s}), 1213(\mathrm{w}), 1147(\mathrm{~s}), 1109$ (m), $1075(\mathrm{w}), 1075(\mathrm{w}), 999(\mathrm{w}), 958(\mathrm{~m}), 877(\mathrm{w}), 768(\mathrm{~s}), 762$ (s), $744(\mathrm{~s}), 733(\mathrm{~s}), 700(\mathrm{~m})$; HRMS (ESI+/TOF) $m / z[\mathrm{M}+\mathrm{H}]^{+}$ calcd for $\mathrm{C}_{15} \mathrm{H}_{12} \mathrm{~N}_{2} \mathrm{Br}$ 299.0184, 301.0164, found 299.0180, 301.0167; mp 169.2-172.5 (EtOAc) (lit. $169-171{ }^{\circ} \mathrm{C}$ ), turned brown upon heating. Spectroscopic data are in accordance with the literature. ${ }^{28,30}$

3-(5-Phenyl-1H-imidazol-2-yl)phenol (16). The title compound was prepared according to general procedure $\mathrm{B}$, using acetophenone (46.0 mg, $0.375 \mathrm{mmol}, 1.25$ equiv) and 3-hydroxybenzaldehyde ( 37.0 $\mathrm{mg}, 0.30 \mathrm{mmol}, 1.00$ equiv). Purification by silica gel chromatography, eluting with EtOAc in hexanes $(19 \mathrm{~cm} \times 20 \mathrm{~mm}$, gradient elution, $0 \%$ $\rightarrow 30 \%, 5 \%$ increases, $50 \mathrm{~mL}$ runs, $5-10 \mathrm{~mL}$ fractions), yielded $\mathbf{1 6}$ as a white yellow solid ( $85 \%$ yield, $60.0 \mathrm{mg}, 0.25 \mathrm{mmol}): R_{f}=0.30(30 \%$ EtOAc/hexane, UV, Dragendorff stain); ${ }^{1} \mathrm{H}$ NMR $(400 \mathrm{MHz}$, $\left.\mathrm{MeOD}-d_{4}\right) \delta 7.76(\mathrm{dd}, J=1.1,8.3 \mathrm{~Hz}, 2 \mathrm{H}), 7.44(\mathrm{~s}, 1 \mathrm{H}), 7.41-$ $7.36(\mathrm{~m}, 4 \mathrm{H}), 7.30-7.23(\mathrm{~m}, 2 \mathrm{H}), 6.83(\mathrm{ddd}, J=1.1,2.4,8.0 \mathrm{~Hz}$, $1 \mathrm{H}) ;{ }^{13} \mathrm{C}\left\{{ }^{1} \mathrm{H}\right\}$ NMR $\left(126 \mathrm{MHz}, \mathrm{MeOD}-d_{4}\right) \delta 159.1,149.0,132.7$, $131.0,129.7,128.0,126.1,117.9,116.9,113.7$ (note that, due to slow relaxation, some ${ }^{13} \mathrm{C}\left\{{ }^{1} \mathrm{H}\right\}$ NMR signals were difficult to identify; ${ }^{28}$ concerning this compound, three signals are missing); HRMS (ESI +/TOF) $m / z[\mathrm{M}+\mathrm{H}]^{+}$calcd for $\mathrm{C}_{15} \mathrm{H}_{13} \mathrm{~N}_{2} \mathrm{O}$ 237.1028, found 237.1011.

2-(3-Chlorophenyl)-5-phenyl-1H-imidazole (17). The title compound was prepared according to general procedure B, using acetophenone (46.0 mg, $0.375 \mathrm{mmol}, 1.25$ equiv) and 3chlorobenzaldehyde ( $42.0 \mathrm{mg}, 0.30 \mathrm{mmol}, 1.00$ equiv). Purification by silica gel chromatography, eluting with EtOAc in hexanes $(19 \mathrm{~cm} \times$ $20 \mathrm{~mm}$, gradient elution, $0 \% \rightarrow 30 \%, 5 \%$ increases, $50 \mathrm{~mL}$ runs, $5-10$ $\mathrm{mL}$ fractions), yielded 17 as a pale yellow solid (69\% yield, $53.0 \mathrm{mg}$, $0.21 \mathrm{mmol}): R_{f}=0.40$ (30\% EtOAc/hexane, UV, Dragendorff stain); ${ }^{1} \mathrm{H}$ NMR $\left(500 \mathrm{MHz}, \mathrm{DMSO}-d_{6}\right) \delta 12.80(\mathrm{~s}, 1 \mathrm{H}), 8.06(\mathrm{~s}, 1 \mathrm{H}), 7.97$ 
$(\mathrm{d}, J=7.5 \mathrm{~Hz}, 1 \mathrm{H}), 7.91-7.76(\mathrm{~m}, 3 \mathrm{H}), 7.50(\mathrm{t}, J=7.9 \mathrm{~Hz}, 1 \mathrm{H})$, 7.42 (ddd, $J=8.0,2.1,0.9 \mathrm{~Hz}, 1 \mathrm{H}), 7.38(\mathrm{t}, J=7.3 \mathrm{~Hz}, 2 \mathrm{H}), 7.22(\mathrm{t}, J$ $=6.9 \mathrm{~Hz}, 1 \mathrm{H}) ;{ }^{13} \mathrm{C}\left\{{ }^{1} \mathrm{H}\right\}$ NMR $\left(126 \mathrm{MHz}\right.$, DMSO- $\left.d_{6}\right) \delta 144.4,141.4$, $133.6,132.5,130.7,128.9,128.5,127.8,127.0,126.4,124.4,123.4$, 114.9; HRMS (ESI+/TOF) $m / z[\mathrm{M}+\mathrm{H}]^{+}$calcd for $\mathrm{C}_{15} \mathrm{H}_{12} \mathrm{ClN}_{2}$ $255.0689,257.0663$, found $255.0693,257.0670$. Spectroscopic data are in accordance with the literature. ${ }^{23}$

2-(4-Nitrophenyl)-5-phenyl-1H-imidazole (18). The title compound was prepared according to general procedure B, using acetophenone (46.0 mg, $0.375 \mathrm{mmol}, 1.25$ equiv) and 4-nitrobenzaldehyde ( $46.0 \mathrm{mg}, 0.30 \mathrm{mmol}, 1.00$ equiv). Purification by silica gel chromatography, eluting with EtOAc in hexanes $(19 \mathrm{~cm} \times 20 \mathrm{~mm}$, gradient elution, $0 \% \rightarrow 30 \%$, $5 \%$ increases, $50 \mathrm{~mL}$ runs, $5-10 \mathrm{~mL}$ fractions), yielded 18 as an orange solid (66\% yield, $53.0 \mathrm{mg}, 0.21$ mmol): $R_{f}=0.20$ (30\% EtOAc/hexane, Dragendorff stain); ${ }^{1} \mathrm{H}$ NMR $\left(500 \mathrm{MHz}, \mathrm{DMSO}-d_{6}\right) \delta 13.13(\mathrm{~s}, 1 \mathrm{H}), 8.34(\mathrm{~d}, J=8.9 \mathrm{~Hz}, 2 \mathrm{H}), 8.23$ $(\mathrm{d}, J=8.8 \mathrm{~Hz}, 2 \mathrm{H}), 7.93(\mathrm{~s}, 1 \mathrm{H}), 7.89(\mathrm{~d}, J=7.6 \mathrm{~Hz}, 2 \mathrm{H}), 7.40(\mathrm{t}, J=$ $7.5 \mathrm{~Hz}, 2 \mathrm{H}), 7.24(\mathrm{t}, J=7.3 \mathrm{~Hz}, 1 \mathrm{H}) ;{ }^{13} \mathrm{C}\left\{{ }^{1} \mathrm{H}\right\} \mathrm{NMR}(63 \mathrm{MHz}$, DMSO- $\left.d_{6}\right) \delta 146.5,143.8,142.4,136.3,134.1,128.5,126.7,125.5$, 124.5, 124.3, 116.3; HRMS (ESI+/TOF) $\mathrm{m} / z[\mathrm{M}+\mathrm{H}]^{+}$calcd for $\mathrm{C}_{15} \mathrm{H}_{12} \mathrm{~N}_{3} \mathrm{O}_{2}$ 266.0930, found 266.0933. Spectroscopic data are in accordance with the literature. ${ }^{32}$

4-(4-Phenyl-1H-imidazol-2-yl)phenol (19). The title compound was prepared according to general procedure $\mathrm{B}$, using acetophenone ( $46.0 \mathrm{mg}, 0.375 \mathrm{mmol}, 1.25$ equiv) and 4-hydroxybenzaldehyde ( 37.0 $\mathrm{mg}, 0.30 \mathrm{mmol}, 1.00$ equiv). Purification by silica gel chromatography, eluting with EtOAc in hexanes $(19 \mathrm{~cm} \times 20 \mathrm{~mm}$, gradient elution, $0 \%$ $\rightarrow 30 \%$, $5 \%$ increases, $50 \mathrm{~mL}$ runs, $5-10 \mathrm{~mL}$ fractions), yielded 19 as a yellow solid ( $50 \%$ yield, $36.0 \mathrm{mg}, 0.15 \mathrm{mmol}): R_{f}=0.30(30 \%$ EtOAc/hexane, Dragendorff stain); ${ }^{1} \mathrm{H}$ NMR $\left(250 \mathrm{MHz}\right.$, DMSO- $\left.d_{6}\right)$ $\delta 12.34(\mathrm{~s}, 1 \mathrm{H}), 9.68(\mathrm{~s}, 1 \mathrm{H}), 7.90-7.71(\mathrm{~m}, 4 \mathrm{H}), 7.65(\mathrm{~s}, 1 \mathrm{H}), 7.35$ $(\mathrm{t}, J=7.6 \mathrm{~Hz}, 2 \mathrm{H}), 7.18(\mathrm{t}, J=7.2 \mathrm{~Hz}, 1 \mathrm{H}), 6.84(\mathrm{~d}, J=8.5 \mathrm{~Hz}, 2 \mathrm{H})$; ${ }^{13} \mathrm{C}\left\{{ }^{1} \mathrm{H}\right\}$ NMR (63 MHz, DMSO- $\left.d_{6}\right) \delta 157.6,146.4,140.5,134.9$, $128.4,126.5,126.0,124.3,122.0,115.4,113.3 ; \nu_{\max }\left(\mathrm{cm}^{-1}\right.$, thin film, ATR) 3221, 2926, 1773,1701, 1609, 1541, 1496, 1460, 1367, 1275, 1175, 1099, 1029, 948, 908, 837, 761, 738, 694, 661, 635; HRMS (ESI+/TOF) $m / z[\mathrm{M}+\mathrm{H}]^{+}$calcd for $\mathrm{C}_{15} \mathrm{H}_{13} \mathrm{~N}_{2} \mathrm{O}$ 237.1028, found 237.1013; mp $227^{\circ} \mathrm{C}$ (dec).

2-(3-Chlorophenyl)-5-phenyl-1H-imidazole (20). The title compound was prepared according to general procedure B, using acetophenone (46.0 $\mathrm{mg}, 0.375 \mathrm{mmol}, 1.25$ equiv) and 4chlorobenzaldehyde ( $42.0 \mathrm{mg}, 0.30 \mathrm{mmol}, 1.00$ equiv). Purification by silica gel chromatography, eluting with EtOAc in hexanes $(19 \mathrm{~cm} \times$ $20 \mathrm{~mm}$, gradient elution, $0 \% \rightarrow 30 \%, 5 \%$ increases, $50 \mathrm{~mL}$ runs, $5-10$ $\mathrm{mL}$ fractions), yielded 20 as a pale yellow solid (72\% yield, $55.0 \mathrm{mg}$, $0.22 \mathrm{mmol}): R_{f}=0.40$ (30\% EtOAc/hexane, UV, Dragendorff stain); ${ }^{1} \mathrm{H}$ NMR $\left(500 \mathrm{MHz}, \mathrm{DMSO}-d_{6}\right) \delta 12.74(\mathrm{~s}, 1 \mathrm{H}), 8.01(\mathrm{~d}, J=8.5 \mathrm{~Hz}$, $2 \mathrm{H}), 7.86(\mathrm{~d}, J=7.4 \mathrm{~Hz}, 2 \mathrm{H}), 7.79(\mathrm{~d}, J=1.8 \mathrm{~Hz}, 1 \mathrm{H}), 7.54(\mathrm{~d}, J=$ $8.5 \mathrm{~Hz}, 2 \mathrm{H}), 7.37(\mathrm{t}, J=7.7 \mathrm{~Hz}, 2 \mathrm{H}), 7.21(\mathrm{t}, J=7.3 \mathrm{~Hz}, 1 \mathrm{H})$; ${ }^{13} \mathrm{C}\left\{{ }^{1} \mathrm{H}\right\}$ NMR $\left(126 \mathrm{MHz}\right.$, DMSO- $\left.d_{6}\right) \delta 144.8,141.3,134.5,132.6$, $129.4,128.8,128.4,126.5,126.3,124.4,114.7$; HRMS (ESI+/TOF) $m / z[\mathrm{M}+\mathrm{H}]^{+}$calcd for $\mathrm{C}_{15} \mathrm{H}_{12} \mathrm{ClN}_{2}$ 255.0689, 257.0663, found 255.0694, 257.0677. Spectroscopic data are in accordance with the literature. ${ }^{32}$

2-(3-Nitrophenyl)-5-phenyl-1H-imidazole (21). The title compound was prepared according to general procedure B, using acetophenone (46.0 mg, $0.375 \mathrm{mmol}, 1.25$ equiv) and 3-nitrobenzaldehyde ( $46.0 \mathrm{mg}, 0.30 \mathrm{mmol}, 1.00$ equiv). Purification by silica gel chromatography, eluting with EtOAc in hexanes $(19 \mathrm{~cm} \times 20 \mathrm{~mm}$, gradient elution, $0 \% \rightarrow 30 \%$, $5 \%$ increases, $50 \mathrm{~mL}$ runs, $5-10 \mathrm{~mL}$ fractions), yielded 21 as a yellow solid ( $72 \%$ yield, $53.0 \mathrm{mg}, 0.21$ $\mathrm{mmol}): R_{f}=0.20$ (30\% EtOAc/hexane, Dragendorff stain); ${ }^{1} \mathrm{H}$ NMR $\left(250 \mathrm{MHz}, \mathrm{DMSO}-d_{6}\right) \delta 13.06(\mathrm{~s}, 1 \mathrm{H}), 8.84(\mathrm{~s}, 1 \mathrm{H}), 8.43(\mathrm{~d}, J=7.8$ $\mathrm{Hz}, 1 \mathrm{H}), 8.20(\mathrm{dd}, J=8.1,2.1 \mathrm{~Hz}, 1 \mathrm{H}), 7.93-7.91(\mathrm{~m}, 4 \mathrm{H}), 7.39(\mathrm{t}, J$ $=7.5 \mathrm{~Hz}, 2 \mathrm{H}), 7.24(\mathrm{t}, J=7.0 \mathrm{~Hz}, 1 \mathrm{H}) ;{ }^{13} \mathrm{C}\left\{{ }^{1} \mathrm{H}\right\} \mathrm{NMR}(63 \mathrm{MHz}$, DMSO- $\left.d_{6}\right) \delta 148.4,143.8,141.7,134.2,132.1,130.9,130.5,128.5$, 126.5, 124.5, 122.5, 119.2, 115.4; HRMS (ESI+/TOF) $m / z[\mathrm{M}+\mathrm{H}]^{+}$ calcd for $\mathrm{C}_{15} \mathrm{H}_{12} \mathrm{~N}_{3} \mathrm{O}_{2}$ 266.0930, found 266.0950 .

2-(4-Methoxyphenyl)-5-phenyl-1H-imidazole (22). The title compound was prepared according to general procedure $\mathrm{B}$, using acetophenone ( $46.0 \mathrm{mg}, 0.375 \mathrm{mmol}, 1.25$ equiv) and $p$-anisaldehyde $(41.0 \mathrm{mg}, 0.30 \mathrm{mmol}, 1.00$ equiv). Purification by silica gel chromatography, eluting with EtOAc in hexanes $(19 \mathrm{~cm} \times 20 \mathrm{~mm}$, gradient elution, $0 \% \rightarrow 40 \%, 5 \%$ increases, $50 \mathrm{~mL}$ runs, $5-10 \mathrm{~mL}$ fractions), yielded 22 as a white solid ( $50 \%$ yield, $38.0 \mathrm{mg}, 0.15$ $\mathrm{mmol}): R_{f}=0.30(30 \%$ EtOAc/hexane, UV, Dragendorff stain $) ;{ }^{1} \mathrm{H}$ NMR (400 MHz, DMSO- $\left.d_{6} / \mathrm{D}_{2} \mathrm{O} / \mathrm{TFA}\right) \delta 7.99(\mathrm{~s}, 1 \mathrm{H}), 7.95$ (d, $J=$ $8.8 \mathrm{~Hz}, 2 \mathrm{H}), 7.80(\mathrm{~d}, J=7.6 \mathrm{~Hz}, 2 \mathrm{H}), 7.50(\mathrm{t}, J=7.5 \mathrm{~Hz}, 2 \mathrm{H}), 7.43$ $(\mathrm{t}, J=7.2 \mathrm{~Hz}, 1 \mathrm{H}), 7.16(\mathrm{~d}, J=8.8 \mathrm{~Hz}, 2 \mathrm{H}), 3.82(\mathrm{~s}, 3 \mathrm{H}) ;{ }^{13} \mathrm{C}\left\{{ }^{1} \mathrm{H}\right\}$ NMR $\left(101 \mathrm{MHz}\right.$, DMSO- $\left.d_{6} / \mathrm{D}_{2} \mathrm{O} / \mathrm{TFA}\right) \delta 163.0,145.3,133.9,130.1$, 130.0, 129.8, 127.2, 126.4, 116.3, 115.7, 115.6, 56.3; HRMS (ESI +/TOF) $m / z[\mathrm{M}+\mathrm{H}]^{+}$calcd for $\mathrm{C}_{16} \mathrm{H}_{15} \mathrm{~N}_{2} \mathrm{O}$ 251.1184, found 251.1186

2-(2-Chlorophenyl)-5-phenyl-1H-imidazole (23). The title compound was prepared according to general procedure B, using acetophenone (46.0 mg, $0.375 \mathrm{mmol}, 1.25$ equiv) and 2chlorobenzaldehyde $(42.0 \mathrm{mg}, 0.30 \mathrm{mmol}, 1.00$ equiv). Purification by silica gel chromatography, eluting with EtOAc in hexanes $(19 \mathrm{~cm} \times$ $20 \mathrm{~mm}$, gradient elution, $0 \% \rightarrow 30 \%, 5 \%$ increases, $50 \mathrm{~mL}$ runs, $5-10$ $\mathrm{mL}$ fractions), yielded 23 as a white solid (63\% yield, $48.0 \mathrm{mg}, 0.19$ mmol): $R_{f}=0.50$ (30\% EtOAc/hexane, UV, Dragendorff stain); ${ }^{1} \mathrm{H}$ NMR $\left(500 \mathrm{MHz}\right.$, DMSO- $\left.d_{6}\right) \delta 12.43(\mathrm{~s}, 1 \mathrm{H}), 7.78(\mathrm{~d}, J=2.0 \mathrm{~Hz}$, $1 \mathrm{H}), 7.88-7.84(\mathrm{~m}, 3 \mathrm{H}), 7.58(\mathrm{dd}, J=1.9,7.2 \mathrm{~Hz}, 1 \mathrm{H}), 7.48-7.43$ $(\mathrm{m}, 2 \mathrm{H}), 7.37(\mathrm{t}, J=7.6 \mathrm{~Hz}, 2 \mathrm{H}), 7.21(\mathrm{t}, J=7.3 \mathrm{~Hz}, 1 \mathrm{H}) ;{ }^{13} \mathrm{C}\left\{{ }^{1} \mathrm{H}\right\}$ NMR $\left(126 \mathrm{MHz}\right.$, DMSO- $\left.d_{6}\right) \delta 143.3,140.8,134.5,131.2,130.8$, $130.2,130.0,129.9,128.4,127.3,126.3,124.4,114.5 ; \nu_{\max }\left(\mathrm{cm}^{-1}\right.$, thin film, ATR) 3059, 1708, 1607, 1567, 1482, 1453, 1111, 1086, 1049, 946, 694; HRMS (ESI+/TOF) $m / z[\mathrm{M}+\mathrm{H}]^{+}$calcd for $\mathrm{C}_{15} \mathrm{H}_{12} \mathrm{ClN}_{2}$ 255.0689, 257.0663, found 255.0690, 257.0670; mp 161.0-162.0 ${ }^{\circ} \mathrm{C}$ (EtOAc).

2-(4-Fluorophenyl)-5-phenyl-1H-imidazole (24). The title compound was prepared according to general procedure B, using acetophenone (46.0 $\mathrm{mg}, 0.375 \mathrm{mmol}, 1.25$ equiv) and 4fluorobenzaldehyde $(38.0 \mathrm{mg}, 0.30 \mathrm{mmol}, 1.00$ equiv). Purification by silica gel chromatography, eluting with EtOAc in hexanes $(19 \mathrm{~cm} \times$ $20 \mathrm{~mm}$, gradient elution, $0 \% \rightarrow 30 \%$, $5 \%$ increases, $50 \mathrm{~mL}$ runs, $5-10$ $\mathrm{mL}$ fractions), yielded 24 as a pale yellow solid (57\% yield, $41.0 \mathrm{mg}$, $0.17 \mathrm{mmol}): R_{f}=0.30$ (30\% EtOAc/hexane, UV, Dragendorff stain); ${ }^{1} \mathrm{H}$ NMR $\left(500 \mathrm{MHz}\right.$, DMSO- $\left.d_{6}\right) \delta 12.65(\mathrm{~s}, 1 \mathrm{H}), 8.04(\mathrm{dd}, J=8.6$, $5.5 \mathrm{~Hz}, 2 \mathrm{H}), 7.86(\mathrm{~d}, J=7.4 \mathrm{~Hz}, 2 \mathrm{H}), 7.76(\mathrm{~s}, 1 \mathrm{H}), 7.37(\mathrm{t}, J=7.6$ $\mathrm{Hz}, 2 \mathrm{H}), 7.32(\mathrm{t}, J=8.8 \mathrm{~Hz}, 2 \mathrm{H}), 7.21(\mathrm{t}, J=7.3 \mathrm{~Hz}, 1 \mathrm{H}) ;{ }^{13} \mathrm{C}\left\{{ }^{1} \mathrm{H}\right\}$ NMR $\left(126 \mathrm{MHz}, \mathrm{DMSO}-d_{6}\right) \delta 162.0\left(\mathrm{~d},{ }^{1} J_{\mathrm{CF}}=245.1 \mathrm{~Hz}\right), 145.1$, 141.1, 134.6, 128.5, $127.30\left(\mathrm{~d},{ }^{4} J_{\mathrm{CF}}=2.5 \mathrm{~Hz}, 1 \mathrm{C}\right), 127.04\left(\mathrm{~d},{ }^{3} J_{\mathrm{CF}}=\right.$ $8.4 \mathrm{~Hz}), 126.2,124.4,115.74\left(\mathrm{~d},{ }^{2} J_{\mathrm{CF}}=22.1 \mathrm{~Hz}\right), 114.3$; HRMS (ESI +/TOF) $m / z[\mathrm{M}+\mathrm{H}]^{+}$calcd for $\mathrm{C}_{15} \mathrm{H}_{12} \mathrm{FN}_{2}$ 239.0984, found 239.0985. Spectroscopic data are in accordance with the literature. ${ }^{33}$

$\mathrm{N}, \mathrm{N}$-Dimethyl-4-(5-phenyl-1H-imidazol-2-yl)aniline (25). The title compound was prepared according to general procedure $\mathrm{B}$, using acetophenone (46.0 $\mathrm{mg}, 0.375 \mathrm{mmol}, 1.25$ equiv) and 4(dimethylamino)benzaldehyde ( $46.0 \mathrm{mg}, 0.30 \mathrm{mmol}, 1.00$ equiv). Purification by silica gel chromatography, eluting with EtOAc in hexanes $(19 \mathrm{~cm} \times 20 \mathrm{~mm}$, gradient elution, $30 \% \rightarrow 50 \%$, $5 \%$ increases, $50 \mathrm{~mL}$ runs, $5-10 \mathrm{~mL}$ fractions), yielded 25 as a yellow solid ( $23 \%$ yield, $18.0 \mathrm{mg}, 0.07 \mathrm{mmol}$ ): $R_{f}=0.40$ (30\% EtOAc/ hexane, UV, Dragendorff stain); ${ }^{1} \mathrm{H}$ NMR $\left(400 \mathrm{MHz}\right.$, DMSO- $d_{6} /$ $\left.\mathrm{D}_{2} \mathrm{O} / \mathrm{TFA}\right) \delta 7.88(\mathrm{~s}, 1 \mathrm{H}), 7.83(\mathrm{~d}, J=9.1 \mathrm{~Hz}, 2 \mathrm{H}), 7.77(\mathrm{~d}, J=7.1$ $\mathrm{Hz}, 1 \mathrm{H}), 7.49(\mathrm{t}, J=7.5 \mathrm{~Hz}, 2 \mathrm{H}), 7.42(\mathrm{t}, J=7.4 \mathrm{~Hz}, 1 \mathrm{H}), 6.85(\mathrm{~d}, J=$ $9.2 \mathrm{~Hz}, 2 \mathrm{H}), 2.98(\mathrm{~s}, 6 \mathrm{H}) ;{ }^{13} \mathrm{C}\left\{{ }^{1} \mathrm{H}\right\}$ NMR (101 MHz, DMSO-d $d_{6} /$ $\left.\mathrm{D}_{2} \mathrm{O} / \mathrm{TFA}\right) \delta 153.1,146.4,133.3,130.0,129.0,127.5,126.3,115.7$, $112.7,109.5,40.4 ; \nu_{\max }\left(\mathrm{cm}^{-1}\right.$, thin film, ATR) 2919, 2850, 1615, $1545,1500,1443,1396,1363,1227,1202,1170,945,820,760,738$, 695; HRMS (ESI+/TOF) $m / z[\mathrm{M}+\mathrm{H}]^{+}$calcd for $\mathrm{C}_{17} \mathrm{H}_{18} \mathrm{~N}_{3}$ 264,1501, found 264,1502; mp 142.0-145.0 ${ }^{\circ} \mathrm{C}$ (EtOAc).

2-(Furan-2-yl)-5-phenyl-1H-imidazole (26). The title compound was prepared according to general procedure $\mathrm{B}$, using acetophenone (46.0 mg, $0.375 \mathrm{mmol}, 1.25$ equiv) and furfural $(29.0 \mathrm{mg}, 0.30 \mathrm{mmol}$, 1.00 equiv). Purification by silica gel chromatography, eluting with EtOAc in hexanes $(19 \mathrm{~cm} \times 20 \mathrm{~mm}$, gradient elution, $0 \% \rightarrow 20 \%, 5 \%$ increases, $50 \mathrm{~mL}$ runs, $5-10 \mathrm{~mL}$ fractions), yielded 26 as a white solid ( $34 \%$ yield, $22.0 \mathrm{mg}$, $0.11 \mathrm{mmol}$ ): $R_{f}=0.10$ (30\% EtOAc/hexane, UV, 
Dragendorff stain); ${ }^{1} \mathrm{H}$ NMR (400 MHz, DMSO- $\left.d_{6} / \mathrm{D}_{2} \mathrm{O} / \mathrm{TFA}\right) \delta$ $8.00(\mathrm{~s}, 1 \mathrm{H}), 7.99(\mathrm{~s}, 1 \mathrm{H}), 7.78(\mathrm{~d}, J=7.5 \mathrm{~Hz}, 2 \mathrm{H}), 7.50(\mathrm{t}, J=7.5$ $\mathrm{Hz}, 2 \mathrm{H}), 7.46-7.41(\mathrm{~m}, 2 \mathrm{H}), 6.80(\mathrm{dd}, J=3.3,1.5 \mathrm{~Hz}, 1 \mathrm{H}) ;{ }^{13} \mathrm{C}\left\{{ }^{1} \mathrm{H}\right\}$ NMR (101 MHz, DMSO- $\left.d_{6} / \mathrm{D}_{2} \mathrm{O} / \mathrm{TFA}\right) \delta 147.8,138.8,136.7,134.0$, 130.3, 130.1, 127.1, 126.3, 116.4, 115.5, 113.9; HRMS (ESI+/TOF) $m / z[\mathrm{M}+\mathrm{H}]^{+}$calcd for $\mathrm{C}_{13} \mathrm{H}_{11} \mathrm{~N}_{2} \mathrm{O}$ 211.0871, found 211.0871. Spectroscopic data are in accordance with the literature ${ }^{34}$

2-(4-Bromophenyl)-5-phenyl-1H-imidazole (27). The title compound was prepared according to general procedure B, using acetophenone (46.0 $\mathrm{mg}, 0.375 \mathrm{mmol}, 1.25$ equiv) and 4bromobenzaldehyde $(56.0 \mathrm{mg}, 0.30 \mathrm{mmol}, 1.00$ equiv). Purification by silica gel chromatography, eluting with EtOAc in hexanes $(19 \mathrm{~cm} \times$ $20 \mathrm{~mm}$, gradient elution, $0 \% \rightarrow 30 \%, 5 \%$ increases, $50 \mathrm{~mL}$ runs, $5-10$ $\mathrm{mL}$ fractions), yielded 27 as a white solid (61\% yield, $55.0 \mathrm{mg}, 0.18$ $\mathrm{mmol}): R_{f}=0.20$ (30\% EtOAc/hexane, UV, Dragendorff stain); ${ }^{1} \mathrm{H}$ NMR $\left(500 \mathrm{MHz}, \mathrm{DMSO}-d_{6}\right) \delta 12.75(\mathrm{~s}, 1 \mathrm{H}), 7.94(\mathrm{~d}, J=8.5 \mathrm{~Hz}$, $2 \mathrm{H}), 7.86(\mathrm{~d}, J=7.2 \mathrm{~Hz}, 2 \mathrm{H}), 7.79(\mathrm{~s}, 1 \mathrm{H}), 7.67(\mathrm{~d}, J=8.5 \mathrm{~Hz}, 2 \mathrm{H})$, $7.37(\mathrm{t}, J=7.7 \mathrm{~Hz}, 2 \mathrm{H}), 7.21(\mathrm{t}, J=7.3 \mathrm{~Hz}, 1 \mathrm{H}) ;{ }^{13} \mathrm{C}\left\{{ }^{1} \mathrm{H}\right\} \operatorname{NMR}(126$ MHz, DMSO- $\left.d_{6}\right) \delta 144.8,141.3,134.5,131.7,129.8,128.5,126.9$, $126.3,124.4,121.2,114.7 ; \nu_{\max }\left(\mathrm{cm}^{-1}\right.$, thin film, ATR) 3069, 1703, $1603,1486,1466,1452,1431,1364,1298,1269,1228,1143,1085$, 1971, 1010, 949, 911, 830, 729, 694; HRMS (ESI+/TOF) $\mathrm{m} / z[\mathrm{M}+$ $\mathrm{H}]^{+}$calcd for $\mathrm{C}_{15} \mathrm{H}_{12} \mathrm{BrN}_{2} 299.0184,301.0164$, found 299.0186, 301.0171; mp 196.0-198.0 ${ }^{\circ} \mathrm{C}$ (EtOAc).

2-Cyclohexyl-5-phenyl-1H-imidazole (28). The title compound was prepared according to general procedure $\mathrm{B}$, using acetophenone (46.0 mg, $0.375 \mathrm{mmol}, 1.25$ equiv) and cyclohexanecarboxaldehyde ( $34.0 \mathrm{mg}, 0.30 \mathrm{mmol}, 1.00$ equiv). Purification by silica gel chromatography, eluting with EtOAc in hexanes $(19 \mathrm{~cm} \times 20 \mathrm{~mm}$, gradient elution, $0 \% \rightarrow 40 \%, 5 \%$ increases, $50 \mathrm{~mL}$ runs, $5-10 \mathrm{~mL}$ fractions), yielded 28 as a white solid ( $83 \%$ yield, $56.0 \mathrm{mg}, 0.25$ $\mathrm{mmol}): R_{f}=0.20$ (30\% EtOAc/hexane, UV, Dragendorff stain); ${ }^{1} \mathrm{H}$ NMR $\left(500 \mathrm{MHz}, \mathrm{CDCl}_{3}\right) \delta 8.35(\mathrm{br} \mathrm{s}, 1 \mathrm{H}), 7.66(\mathrm{~d}, J=7.3 \mathrm{~Hz}, 2 \mathrm{H})$, $7.32(\mathrm{t}, J=7.7 \mathrm{~Hz}, 2 \mathrm{H}), 7.22-7.17(\mathrm{~m}, 2 \mathrm{H}), 2.74(\mathrm{tt}, J=12.0,3.5 \mathrm{~Hz}$, $1 \mathrm{H}), 1.98(\mathrm{~d}, J=11.7 \mathrm{~Hz}, 2 \mathrm{H}), 1.75(\mathrm{~d}, J=13.2 \mathrm{~Hz}, 2 \mathrm{H}), 1.66(\mathrm{~d}, J=$ $12.6 \mathrm{~Hz}, 1 \mathrm{H}), 1.50(\mathrm{dq}, J=12.4,3.1 \mathrm{~Hz}, 2 \mathrm{H}), 1.32-1.12(\mathrm{~m}, 3 \mathrm{H})$; ${ }^{13} \mathrm{C}\left\{{ }^{1} \mathrm{H}\right\}$ NMR $\left(126 \mathrm{MHz}, \mathrm{CDCl}_{3}\right) \delta 153.9,137.1,133.1,128.7$, $126.7,124.9,115.7,38.1,32.2,26.2$, 25.9; HRMS (ESI+/TOF) $\mathrm{m} / z$ $[\mathrm{M}+\mathrm{H}]^{+}$calcd for $\mathrm{C}_{15} \mathrm{H}_{19} \mathrm{~N}_{2}$ 227.1548, found 227.1558. Spectroscopic data are in accordance with the literature. ${ }^{33-35}$

4-(5-Phenyl-1H-imidazol-2-yl)pyridine (29). The title compound was prepared according to general procedure $\mathrm{B}$, using acetophenone (46.0 mg, $0.375 \mathrm{mmol}, 1.25$ equiv) and 4-pyridinecarboxaldehyde (33.0 mg, $0.30 \mathrm{mmol}, 1.00$ equiv). Purification by silica gel chromatography, eluting with EtOAc in hexanes $(19 \mathrm{~cm} \times 20 \mathrm{~mm}$, gradient elution, $50 \% \rightarrow 100 \%, 5 \%$ increases, $50 \mathrm{~mL}$ runs, $5-10 \mathrm{~mL}$ fractions), yielded 29 as a pale yellow solid ( $40 \%$ yield, $26.5 \mathrm{mg}, 0.12$ mmol): $R_{f}=0.01$ (30\% EtOAc/hexane, UV, Dragendorff stain); ${ }^{1} \mathrm{H}$ NMR (400 MHz, DMSO- $\left.d_{6} / \mathrm{D}_{2} \mathrm{O} / \mathrm{TFA}\right) \delta 8.81(\mathrm{~d}, J=6.6 \mathrm{~Hz}, 2 \mathrm{H})$, $8.40(\mathrm{~d}, J=6.6 \mathrm{~Hz}, 2 \mathrm{H}), 8.04(\mathrm{~s}, 1 \mathrm{H}), 7.85(\mathrm{~d}, J=7.6 \mathrm{~Hz}, 2 \mathrm{H}), 7.45$ $(\mathrm{t}, J=7.6 \mathrm{~Hz}, 2 \mathrm{H}), 7.34(\mathrm{t}, J=7.3 \mathrm{~Hz}, 1 \mathrm{H}) ;{ }^{13} \mathrm{C}\left\{{ }^{1} \mathrm{H}\right\}$ NMR $(101$ $\left.\mathrm{MHz}, \mathrm{DMSO}-d_{6} / \mathrm{D}_{2} \mathrm{O} / \mathrm{TFA}\right) \delta 143.5,143.2,142.0,141.6,131.4$, $129.8,129.1,125.9,122.2$, 122.0; HRMS (ESI+/TOF) $m / z[\mathrm{M}+\mathrm{H}]^{+}$ calcd for $\mathrm{C}_{14} \mathrm{H}_{12} \mathrm{~N}_{3} 222.1031$, found 222.1030. Spectroscopic data are in accordance with the literature. ${ }^{23}$

5-Phenyl-2-propyl-1H-imidazole (30). The title compound was prepared according to general procedure $\mathrm{B}$, using acetophenone (46.0 $\mathrm{mg}, 0.375 \mathrm{mmol}$, 1.25 equiv) and butyraldehyde (23.0 mg, 0.30 mmol, 1.00 equiv). Purification by silica gel chromatography, eluting with EtOAc in hexanes $(19 \mathrm{~cm} \times 20 \mathrm{~mm}$, gradient elution, $30 \% \rightarrow$ $70 \%, 5 \%$ increases, $50 \mathrm{~mL}$ runs, $5-10 \mathrm{~mL}$ fractions), yielded 30 as a white solid ( $36 \%$ yield, $20.0 \mathrm{mg}, 0.11 \mathrm{mmol}): R_{f}=0.10(30 \% \mathrm{EtOAc})$ hexane, UV, Dragendorff stain); ${ }^{1} \mathrm{H}$ NMR $\left(500 \mathrm{MHz}, \mathrm{CDCl}_{3}\right) \delta 7.84$ $(\mathrm{s}, 1 \mathrm{H}), 7.67(\mathrm{~d}, J=7.4 \mathrm{~Hz}, 2, \mathrm{H}), 7.33(\mathrm{t}, J=7.7 \mathrm{~Hz}, 2 \mathrm{H}), 7.23-7.17$ $(\mathrm{m}, 2 \mathrm{H}), 2.68(\mathrm{t}, J=7.6 \mathrm{~Hz}, 2 \mathrm{H}), 1.70(\mathrm{sx}, J=7.5 \mathrm{~Hz}, 2 \mathrm{H}), 0.89(\mathrm{t}, J$ $=7.4 \mathrm{~Hz}, 3 \mathrm{H}) ;{ }^{13} \mathrm{C}\left\{{ }^{1} \mathrm{H}\right\}$ NMR $\left(126 \mathrm{MHz}, \mathrm{CDCl}_{3}\right) \delta 149.7,137.6$, $133.1,128.8,126.8,124.8,115.5,30.6,22.2,13.9$; HRMS (ESI +/TOF) $m / z[\mathrm{M}+\mathrm{H}]^{+}$calcd for $\mathrm{C}_{12} \mathrm{H}_{15} \mathrm{~N}_{2}$ 187.1235, found 187.1243. Spectroscopic data are in accordance with the literature. $^{36,37}$
2-Cyclopropyl-5-phenyl-1H-imidazole (31). The title compound was prepared according to general procedure $\mathrm{B}$, using acetophenone (46.0 $\mathrm{mg}, 0.375 \mathrm{mmol}, 1.25$ equiv) and cyclopropanecarboxaldehyde $(23.0 \mathrm{mg}, 0.30 \mathrm{mmol}, 1.00$ equiv). Purification by silica gel chromatography, eluting with EtOAc in hexanes $(19 \mathrm{~cm} \times 20 \mathrm{~mm}$, gradient elution, $0 \% \rightarrow 40 \%$, $5 \%$ increases, $50 \mathrm{~mL}$ runs, $5-10 \mathrm{~mL}$ fractions), yielded 31 as a white solid (70\% yield, $39.0 \mathrm{mg}, 0.21$ $\mathrm{mmol}): R_{f}=0.10\left(30 \% \mathrm{EtOAc} /\right.$ hexane, UV, Dragendorff stain); ${ }^{1} \mathrm{H}$ NMR (400 MHz, DMSO- $\left.d_{6} / \mathrm{D}_{2} \mathrm{O} / \mathrm{TFA}\right) \delta 7.76(\mathrm{~s}, 1 \mathrm{H}), 7.67$ (d, $J=$ $7.4 \mathrm{~Hz}, 2 \mathrm{H}), 7.46(\mathrm{t}, J=7.5 \mathrm{~Hz}, 2 \mathrm{H}), 7.39(\mathrm{t}, J=7.3 \mathrm{~Hz}, 1 \mathrm{H}), 2.30-$ $2.20(\mathrm{~m}, 1 \mathrm{H}), 1.28-1.20(\mathrm{~m}, 2 \mathrm{H}), 1.18-1.11(\mathrm{~m}, 2 \mathrm{H}) ;{ }^{13} \mathrm{C}\left\{{ }^{1} \mathrm{H}\right\}$ NMR $\left(101 \mathrm{MHz}, \mathrm{DMSO}-d_{6} / \mathrm{D}_{2} \mathrm{O} / \mathrm{TFA}\right) \delta 150.9,132.5,130.0,129.9$, $127.3,125.9,114.7,9.7,7.5 ; \nu_{\max }\left(\mathrm{cm}^{-1}\right.$, thin film, ATR) 3034, 2910, $1606,1566,1545,1524,1483,1451,1425,1313,1166,1135,1090$, $1027,1005,881,756,727,693$; HRMS (ESI+/TOF) $m / z[\mathrm{M}+\mathrm{H}]^{+}$ calcd for $\mathrm{C}_{12} \mathrm{H}_{13} \mathrm{~N}_{2}$ 185.1079, found 185.1080; mp $160.0-162.0{ }^{\circ} \mathrm{C}$ (EtOAc).

4-(2-(2-Methyl-4-(methylsulfonyl)phenyl)-1H-imidazol-5-yl)pyridine (32). A $10 \mathrm{~mL}$ round-bottom flask was charged with 4acetylpyridine (68) (219 $\mathrm{mg}, 1.70 \mathrm{mmol}, 1.70$ equiv), a magnetic stir bar, and DMSO $(3.5 \mathrm{~mL}, 0.5 \mathrm{M})$ under air, and concentrated aq $\mathrm{HBr}$ $(48 \% \mathrm{w} / \mathrm{w}, 8.9 \mathrm{M})(595 \mathrm{~mL}, 5.25 \mathrm{mmol}, 3.0$ equiv) was added dropwise. The reaction mixture was stirred in a preheated oil bath at $60{ }^{\circ} \mathrm{C}$ for $8 \mathrm{~h}$. After consumption of the starting material, indicated by TLC analysis (EtOAc, $p$-ASD), the reaction mixture was left to reach room temperature and $\mathrm{MeOH}(5.7 \mathrm{~mL}, 0.19 \mathrm{M})$ was added. This reaction mixture was added dropwise over $30 \mathrm{~min}$ via syringe to a solution of 2-methyl-4-(methylsulfonyl)benzaldehyde (S5) $(198 \mathrm{mg}$, $1.00 \mathrm{mmol}, 1.00$ equiv) and $\mathrm{NH}_{4} \mathrm{OAc}(771 \mathrm{mg}, 10.0 \mathrm{mmol}, 10.0$ equiv) in $\mathrm{MeOH}(5 \mathrm{~mL}, 0.2 \mathrm{M}$ in relation to $\mathrm{S5}$ ) at room temperature. The reaction mixture was stirred at room temperature for $18 \mathrm{~h}$, and the solvent was removed in the rotaevaporator; the residue was diluted with $10 \% \mathrm{MeOH} / \mathrm{DCM}(10 \mathrm{~mL})$ and poured into a separatory funnel containing saturated $\mathrm{NaHCO}_{3}(1 \times 40 \mathrm{~mL})$ and $10 \% \mathrm{MeOH} / \mathrm{DCM}(1 \times 15 \mathrm{~mL})$. The phases were separated, and the aqueous phase was extracted with $10 \% \mathrm{MeOH} / \mathrm{DCM}(7 \times 10 \mathrm{~mL})$. The organic phases were combined, dried over $\mathrm{MgSO}_{4}$, filtered, and concentrated in the rotaevaporator. Purification by silica gel chromatography, eluting with $\mathrm{MeOH}$ in DCM (gradient elution 5\% $\rightarrow 9 \%$ ), yielded 31 as a pale yellow solid (67\% yield, $210 \mathrm{mg}, 0.67$ mmol): $R_{f}=0.17$ (EtOAc, Dragendorff stain); ${ }^{1} \mathrm{H}$ NMR $(500 \mathrm{MHz}$, DMSO- $\left.d_{6}\right) \delta 12.92(\mathrm{br} \mathrm{s}, 1 \mathrm{H}), 8.54(\mathrm{~d}, J=5.9 \mathrm{~Hz}, 2 \mathrm{H}), 8.14(\mathrm{~s}, 1 \mathrm{H})$, 7.94-7.90 (m, 2H), $7.85(\mathrm{~d}, J=8.8 \mathrm{~Hz}, 1 \mathrm{H}), 7.81(\mathrm{~d}, J=5.9 \mathrm{~Hz}$, $2 \mathrm{H}), 3.26(\mathrm{~s}, 3 \mathrm{H}), 2.75(\mathrm{~s}, 3 \mathrm{H}) ;{ }^{13} \mathrm{C}\left\{{ }^{1} \mathrm{H}\right\}$ NMR $(126 \mathrm{MHz}$, DMSO$\left.d_{6}\right) \delta 149.9,145.4,141.5,140.0,138.8,137.6,134.1,129.6,128.9$, $124.4,118.8,117.7,43.5,21.4 ; \nu_{\max }\left(\mathrm{cm}^{-1}\right.$, ATR): 2673, 1607, 1302, $1150,1106,1077,1004,965,950,828,763,739,709,690$; HRMS (ESI+/TOF) $m / z[\mathrm{M}+\mathrm{H}]^{+}$calcd for $\mathrm{C}_{16} \mathrm{H}_{16} \mathrm{~N}_{3} \mathrm{O}_{2} \mathrm{~S} 314.0963$, found 314.0938; mp 225.4-227.3 ${ }^{\circ} \mathrm{C}(\mathrm{MeOH} / \mathrm{DCM})$.

tert-Butyl 4-(5-(Pyridin-4-yl)-1H-imidazol-2-yl)piperidine-1-carboxylate (36). A $50 \mathrm{~mL}$ round-bottom flask was charged with 4acetylpyridine $(68)(645 \mathrm{mg}, 5.16 \mathrm{mmol}, 1.75$ equiv $)$, a magnetic stir bar, and DMSO $(10.8 \mathrm{~mL}, 0.5 \mathrm{M})$ under air, and concentrated aq $\mathrm{HBr}(48 \% \mathrm{w} / \mathrm{w}, 8.9 \mathrm{M})(1.75 \mathrm{~mL}, 15.5 \mathrm{mmol}, 3.0$ equiv $)$ was added dropwise. The reaction mixture was stirred in a preheated oil bath at $60{ }^{\circ} \mathrm{C}$ for $4 \mathrm{~h}$. After consumption of the starting material, indicated by TLC analysis (EtOAc, $p$-ASD), the reaction mixture was left to reach room temperature and $\mathrm{MeOH}(18.3 \mathrm{~mL}, 0.18 \mathrm{M}$ relative to 4acetylpyridine) was added. This reaction mixture was added dropwise over $30 \mathrm{~min}$ via syringe to a solution of 1-(tert-butoxycarbonyl)-4piperidinecarboxaldehyde (35) (629 $\mathrm{mg}, 2.95 \mathrm{mmol}, 1.00$ equiv) and $\mathrm{NH}_{4} \mathrm{OH}$ (6.4 mL, $44.3 \mathrm{mmol}, 15.0$ equiv) in $\mathrm{MeOH}$ (14.8 mL, $0.2 \mathrm{M}$ in relation to 35) at room temperature. The reaction mixture was stirred at room temperature for $4 \mathrm{~h}$ and poured into a separatory funnel containing saturated $\mathrm{NaHCO}_{3}(1 \times 40 \mathrm{~mL})$ and EtOAc $(1 \times$ $40 \mathrm{~mL})$. The phases were separated, and the aqueous phase was extracted with EtOAc $(3 \times 40 \mathrm{~mL})$. The organic phases were combined, dried over $\mathrm{Na}_{2} \mathrm{SO}_{4}$, filtered, and concentrated in the rotaevaporator. Purification by silica gel chromatography, eluting with $\mathrm{EtOH} / \mathrm{EtOAc} / \mathrm{NH}_{4} \mathrm{OH} /$ hexane $(11: 34: 5: 50)(18 \mathrm{~cm} \times 40 \mathrm{~mm}$, 
isocratic elution, (11:34:5:50) EtOH/EtOAc/ $\mathrm{NH}_{4} \mathrm{OH} /$ hexane, $1 \mathrm{~L}$ run, $20 \mathrm{~mL}$ fractions), yielded 36 as a white solid (82\% yield, $793 \mathrm{mg}$, $2.40 \mathrm{mmol}): R_{f}=0.40\left(\mathrm{EtOH} / \mathrm{EtOAc} / \mathrm{NH}_{4} \mathrm{OH} /\right.$ hexane (11:34:5:50), UV, Dragendorff stain); ${ }^{1} \mathrm{H}$ NMR (500 $\mathrm{MHz}$, DMSO-d $\left.d_{6}\right) \delta 12.10(\mathrm{br} \mathrm{s}, 1 \mathrm{H}), 8.46(\mathrm{~d}, J=6.0 \mathrm{~Hz}, 2 \mathrm{H}), 7.77(\mathrm{br}$ s, $1 \mathrm{H}), 7.66(\mathrm{~d}, J=6.0 \mathrm{~Hz}, 2 \mathrm{H}), 3.99(\mathrm{~d}, J=12.4 \mathrm{~Hz}, 2 \mathrm{H}), 2.96-2.78$ (m, 3H), $1.90(\mathrm{dd}, J=13.0,2.3 \mathrm{~Hz}, 2 \mathrm{H}), 1.59(\mathrm{dq}, J=12.3,3.9 \mathrm{~Hz}$, $2 \mathrm{H}), 1.41(\mathrm{~s}, 9 \mathrm{H}) .{ }^{13} \mathrm{C}\left\{{ }^{1} \mathrm{H}\right\}$ NMR $\left(126 \mathrm{MHz}\right.$, DMSO- $\left.d_{6}\right) \delta 153.9$, 152.1, 149.7, 118.5, 78.6, 43.2, 35.2, 30.4, 28.1 (note that, due to slow relaxation, some ${ }^{13} \mathrm{C}\left\{{ }^{1} \mathrm{H}\right\}$ NMR signals were not identified in the spectra; ${ }^{28}$ specifically, the ${ }^{13} \mathrm{C}\left\{{ }^{1} \mathrm{H}\right\}$ NMR data for compound 36 lacks three of the 12 expected signals); $\nu_{\max }\left(\mathrm{cm}^{-1}\right.$, thin film, ATR) 2867 (br), 1690 (s), 1603 (s), $1553(\mathrm{w}), 1429(\mathrm{~m}), 1363(\mathrm{w}), 1285(\mathrm{w})$, $1248(\mathrm{w}), 1230(\mathrm{w}), 1212(\mathrm{w}), 1173(\mathrm{~s}), 1151(\mathrm{~m}), 1126(\mathrm{~m}), 1038$ (w), $1004(\mathrm{~m}), 942(\mathrm{w}), 876(\mathrm{w}), 766(\mathrm{~s}), 720(\mathrm{w}), 686(\mathrm{~m})$; HRMS (ESI+/TOF) $m / z[\mathrm{M}+\mathrm{H}]^{+}$calcd for $\mathrm{C}_{18} \mathrm{H}_{25} \mathrm{~N}_{4} \mathrm{O}_{2} 329.1978$, found 329.1964; mp $215.0{ }^{\circ} \mathrm{C}$ (dec)

1-(4-Amino-3-bromophenyl)ethenone (43). A $6 \mathrm{~mL}$ vial was charged with the $4^{\prime}$-aminoacetophenone $(51.0 \mathrm{mg}, 0.375 \mathrm{mmol}, 1.00$ equiv), DMSO $(0.75 \mathrm{~mL}, 0.5 \mathrm{M})$, concentrated aqueous $\mathrm{HBr}(48 \%$ $\mathrm{w} / \mathrm{w}, 8.9 \mathrm{M})(47 \mu \mathrm{L}, 0.41 \mathrm{mmol}, 110 \mathrm{~mol} \%)$, deionized water (47 $\mu \mathrm{L}$ ), and a magnetic stir bar under air. The reaction mixture was stirred in a preheated aluminum block at $85{ }^{\circ} \mathrm{C}$ and was followed by TLC analysis (30\% EtOAc/hexane, $p$-ASD). The reaction mixture was poured directly into a separatory funnel containing a mixture of saturated $\mathrm{NaHCO}_{3}$ and saturated $\mathrm{Na}_{2} \mathrm{~S}_{2} \mathrm{O}_{3}(1: 1,1 \times 20 \mathrm{~mL})$ and EtOAc $(10 \mathrm{~mL})$. The phases were separated, and the aqueous phase was extracted with EtOAc $(5 \times 5 \mathrm{~mL})$. The organic phases were combined, washed with saturated $\mathrm{NaCl}$ solution $(1 \times 5 \mathrm{~mL})$, dried over $\mathrm{Na}_{2} \mathrm{SO}_{4}$, filtered, and concentrated in the rotaevaporator. Purification by silica gel chromatography, eluting with EtOAc in hexanes $(18 \mathrm{~cm} \times 15 \mathrm{~mm}$, gradient elution, $0 \% \rightarrow 35 \%, 5 \%$ increases, $30 \mathrm{~mL}$ runs, $10 \mathrm{~mL}$ fractions), yielded 33 as a pale yellow solid (99\% yield, $64.0 \mathrm{mg}, 0.30 \mathrm{mmol}): R_{f}=0.53(30 \% \mathrm{EtOAc} / \mathrm{h}$ exane, $p$-ASD); ${ }^{1} \mathrm{H}$ NMR $\left(500 \mathrm{MHz}, \mathrm{CDCl}_{3}\right) \delta 8.05(\mathrm{~d}, J=1.8 \mathrm{~Hz}, 1 \mathrm{H}), 7.73(\mathrm{dd}, J=$ 8.4, $1.8 \mathrm{~Hz}, 1 \mathrm{H}), 6.74(\mathrm{~d}, J=8.4 \mathrm{~Hz}, 1 \mathrm{H}), 4.60(\mathrm{br} \mathrm{s}, 2 \mathrm{H}), 2.49$ (s, $3 \mathrm{H}) ;{ }^{13} \mathrm{C}\left\{{ }^{1} \mathrm{H}\right\}$ NMR $\left(126 \mathrm{MHz}, \mathrm{CDCl}_{3}\right) \delta 195.5,148.5,133.9,129.5$, $128.9,114.3,108.3,26.2$. Spectroscopic data are in accordance with the literature. $^{38}$

4-(4-Bromo-2-(2-methyl-4-(methylsulfonyl)phenyl)-1H-imidazol5-yl)pyridine (44). Following a modified literature procedure, ${ }^{24}$ a 25 $\mathrm{mL}$ round-bottom flask was charged with $32(595 \mathrm{mg}, 1.90 \mathrm{mmol}$, 1.00 equiv), dry DCM $(8.4 \mathrm{~mL})$, dry pyridine $(2.1 \mathrm{~mL})$, and a magnetic stir bar under an inert atmosphere. The RBF was covered with aluminum foil, and the reaction mixture was cooled to $0{ }^{\circ} \mathrm{C}$ in an ice/water bath and stirred for $15 \mathrm{~min}$. Solid $\mathrm{Py} \cdot \mathrm{HBr} \cdot \mathrm{Br}_{2}$ (pyridinium hydrobromide perbromide, $743 \mathrm{mg}, 2.09 \mathrm{mmol}, 1.10$ equiv) was added in portions, by briefly removing the Suba seal, and the reaction mixture was stirred at $0{ }^{\circ} \mathrm{C}$ for $1 \mathrm{~h}$. After consumption of the starting material, indicated by TLC analysis (100\% EtOAc, Dragendorff), the solvent was removed in the rotaevaporator. The residue was partitioned between $1 \mathrm{M}$ aq $\mathrm{NaHSO}_{3}(1 \times 75 \mathrm{~mL})$ and $10 \%$ $\mathrm{MeOH} / \mathrm{DCM}(1 \times 60 \mathrm{~mL})$. The phases were separated, and the aqueous layer was extracted with $10 \% \mathrm{MeOH} / \mathrm{DCM}(3 \times 60 \mathrm{~mL})$. The organic phases were combined, dried over $\mathrm{MgSO}_{4}$, filtered, and concentrated in the rotaevaporator. The residue was triturated with hexanes, filtered, and washed with hexanes until all pyridine was removed, indicated by TLC analysis, and dried in vacuo to afford 44 as a yellow solid ( $96 \%$ yield, $716 \mathrm{mg}, 1.83 \mathrm{mmol}$ ): $R_{f}=0.47$ (EtOAc, UV, Dragendorff stain); ${ }^{1} \mathrm{H}$ NMR (500 MHz, DMSO- $\left.d_{6}\right) \delta 13.32(\mathrm{~s}$, $1 \mathrm{H}), 8.70(\mathrm{~s}, 2 \mathrm{H}), 8.00-7.79(\mathrm{~m}, 5 \mathrm{H}), 3.28(\mathrm{~s}, 3 \mathrm{H}), 2.65(\mathrm{~s}, 3 \mathrm{H})$; ${ }^{13} \mathrm{C}\left\{{ }^{1} \mathrm{H}\right\}$ NMR $\left(126 \mathrm{MHz}\right.$, DMSO- $\left.d_{6}\right) \delta 150.5,146.7,141.3,138.6$, $136.1,133.7,130.4,129.9,126.6,124.8,120.8,116.0,43.9,21.3 ; \nu_{\max }$ $\left(\mathrm{cm}^{-1}\right.$, thin film, ATR) $2765(\mathrm{br}), 1606(\mathrm{~s}), 1573(\mathrm{w}), 1533(\mathrm{w})$, $1491(\mathrm{w}), 1448(\mathrm{w}), 1422(\mathrm{w}), 1301(\mathrm{~s}), 1222(\mathrm{w}), 1205(\mathrm{w}), 1150$ (s), $1105(\mathrm{~m}), 1077(\mathrm{~m}), 1004(\mathrm{~m}), 986(\mathrm{w}), 964(\mathrm{~m}), 950(\mathrm{~m}), 892$ $(\mathrm{w}), 875(\mathrm{w}), 828(\mathrm{~s}), 762(\mathrm{~s}), 739(\mathrm{~m}), 708(\mathrm{w}), 699(\mathrm{w})$; HRMS (ESI+/TOF) $\mathrm{m} / z[\mathrm{M}+\mathrm{H}]^{+}$calcd for $\mathrm{C}_{16} \mathrm{H}_{15} \mathrm{BrN}_{3} \mathrm{O}_{2} \mathrm{~S} 392.0068$, 394.0049 , found $392.0053,394.0034 ; \mathrm{mp} 225.0{ }^{\circ} \mathrm{C}$ ( dec), turned brown at $210.0^{\circ} \mathrm{C}$.
Suzuki-Miyaura Cross-Coupling: General Procedure C. A culture tube $(13 \mathrm{~mm} \times 100 \mathrm{~mm}, 9 \mathrm{~mL})$ was charged with the corresponding bromo-imidazole $(0.10 \mathrm{mmol}, 1.00$ equiv), corresponding boronic ester or boronic acid $(0.125 \mathrm{mmol}, 1.25$ equiv) and a magnetic stir bar under inert atmosphere. Then, degassed DME (0.5 $\mathrm{mL}$ ) was added followed by addition of a premixed solution of $\mathrm{Pd}(\mathrm{OAc})_{2}(10 \mathrm{~mol} \%)$ and Aphos $(24 \mathrm{~mol} \%)$ in degassed DME $(0.25 \mathrm{~mL})$. The reaction mixture was stirred for $5 \mathrm{~min}$ at room temperature and then $1.2 \mathrm{M}$ aqueous $\mathrm{K}_{2} \mathrm{CO}_{3}(0.25 \mathrm{~mL}, 3.00$ equiv $)$ degassed solution was added and the mixture was stirred for additional $5 \mathrm{~min}$. After this time, the reaction mixture was stirred in a preheated aluminum block at $80{ }^{\circ} \mathrm{C}$ for $18 \mathrm{~h}$. After consumption of the starting material, indicated by TLC analysis $\left(7 \% \mathrm{EtOH} / \mathrm{CHCl}_{3}\right.$, Dragendorff), the reaction mixture was allowed to reach room temperature and it was diluted with $10 \% \mathrm{MeOH} / \mathrm{DCM}(\sim 7 \mathrm{~mL})$, filtered through a pad (20 mm diameter) composed of Celite (top, 1 $\mathrm{cm}$ ) and silica gel (bottom, $3 \mathrm{~cm}$ ). The pad was washed with $10 \%$ $\mathrm{MeOH} / \mathrm{DCM}(25-50 \mathrm{~mL})$ and the filtrate was concentrated under in the reduced pressure. The crude product was adsorbed over basic alumina and purification was performed by silica gel column chromatography.

4-(2-(2-Methyl-4-(methylsulfonyl)phenyl)-4-phenyl-1H-imidazol5-yl)pyridine (46). The title compound was prepared according to general procedure $\mathrm{C}$, using 44 (39.0 $\mathrm{mg}, 0.10 \mathrm{mmol}, 1.00$ equiv), phenylboronic acid (15.0 mg, $0.125 \mathrm{mmol}, 1.25$ equiv), $\mathrm{Pd}(\mathrm{OAc})_{2}$ (2.3 mg, $10 \mathrm{~mol} \%$ ), and Aphos (6.7 mg, $24 \mathrm{~mol} \%)$. Purification by silica gel chromatography, eluting with EtOH in $\mathrm{CHCl}_{3}(21 \mathrm{~cm} \times 10$ $\mathrm{mm}$, gradient elution, $0 \% \rightarrow 4 \%, 0.5 \%$ increases, $20 \mathrm{~mL}$ runs, $3-4 \mathrm{~mL}$ fractions) followed by repurification in silica gel using EtOAc $(4 \mathrm{~cm} \times$ $30 \mathrm{~mm}$, isocratic elution, $100 \% \mathrm{EtOAc}, 150 \mathrm{~mL}$ run, $10 \mathrm{~mL}$ fractions), yielded 46 as a white solid (71\% yield, $28.0 \mathrm{mg}, 0.07 \mathrm{mmol}):{ }^{1} \mathrm{H}$ NMR (500 MHz, DMSO- $\left.d_{6} / \mathrm{D}_{2} \mathrm{O} / \mathrm{TFA}\right) \delta 8.66(\mathrm{~d}, J=7.0 \mathrm{~Hz}, 2 \mathrm{H})$, $8.05(\mathrm{~d}, J=7.0 \mathrm{~Hz}, 2 \mathrm{H}), 7.99(\mathrm{~d}, J=8.2 \mathrm{~Hz} 1 \mathrm{H}), 7.93(\mathrm{~d}, J=1.1 \mathrm{~Hz}$, $2 \mathrm{H}), 7.87(\mathrm{dd}, J=8.2,1.5 \mathrm{~Hz}, 1 \mathrm{H}), 7.66-7.61(\mathrm{~m}, 2 \mathrm{H}), 7.60-7.55$ $(\mathrm{m}, 2 \mathrm{H}), 3.24(\mathrm{~s}, 3 \mathrm{H}), 2.75(\mathrm{~s}, 3 \mathrm{H}) ;{ }^{13} \mathrm{C}\left\{{ }^{1} \mathrm{H}\right\}$ NMR $(126 \mathrm{MHz}$, DMSO- $\left.d_{6} / \mathrm{D}_{2} \mathrm{O} / \mathrm{TFA}\right) \delta 150.9,146.8,141.5,141.1,138.9,137.3$, $133.5,131.5,130.3,130.2,129.9,129.8,129.5,129.5,124.8,122.4$, $43.8,21.5 ; \nu_{\max }\left(\mathrm{cm}^{-1}\right.$, thin film, ATR) $3084(\mathrm{br}), 2928(\mathrm{w}), 1601(\mathrm{~s})$, $1501(\mathrm{w}), 1486(\mathrm{w}), 1444(\mathrm{w}), 1327(\mathrm{~m}), 1303(\mathrm{~s}), 1214(\mathrm{w}), 1147$ (s), $1108(\mathrm{~m}), 1074(\mathrm{~m}), 999(\mathrm{w}), 962(\mathrm{~m}), 951(\mathrm{~m}), 879(\mathrm{w}), 832$ (s), 777 (m), 762 (s), 742 (s), 702 (s); HRMS (ESI+/TOF) $m / z$ [M $+\mathrm{H}]^{+}$calcd for $\mathrm{C}_{22} \mathrm{H}_{20} \mathrm{~N}_{3} \mathrm{O}_{2} \mathrm{~S} 390.1276$, found 390.1273; mp 265.0 ${ }^{\circ} \mathrm{C}(\mathrm{dec})$.

4-(4-(4-(Benzyloxy)phenyl)-2-(2-methyl-4-(methylsulfonyl)phenyl)-1H-imidazol-5-yl)pyridine (47). The title compound was prepared according to general procedure C, using $44(39.0 \mathrm{mg}, 0.10$ mmol, 1.00 equiv), 4-benzyloxyphenylboronic acid $(29.0 \mathrm{mg}, 0.125$ mmol, 1.25 equiv), $\mathrm{Pd}(\mathrm{OAc})_{2}(2.3 \mathrm{mg}, 10 \mathrm{~mol} \%)$, and Aphos (6.7 $\mathrm{mg}, 24 \mathrm{~mol} \%)$. Purification by silica gel chromatography, eluting with EtOH in $\mathrm{CHCl}_{3}(21 \mathrm{~cm} \times 10 \mathrm{~mm}$, gradient elution, $0 \% \rightarrow 6 \%, 0.5 \%$ increases, $20 \mathrm{~mL}$ runs, 3-4 mL fractions) followed by repurification in silica gel using $\mathrm{MeOH}$ in DCM eluent $(21 \mathrm{~cm} \times 10 \mathrm{~mm}$, gradient elution, $0 \% \rightarrow 6 \%, 0.5 \%$ increases, $20 \mathrm{~mL}$ runs, $7 \mathrm{~mL}$ fractions), yielded 47 as a pale yellow solid (69\% yield, $34.0 \mathrm{mg}, 0.07 \mathrm{mmol}):{ }^{1} \mathrm{H}$ NMR (500 MHz, DMSO- $\left.d_{6} / \mathrm{D}_{2} \mathrm{O} / \mathrm{TFA}\right) \delta 8.57(\mathrm{~d}, J=7.0 \mathrm{~Hz}, 2 \mathrm{H})$, $8.04(\mathrm{~d}, J=7.0 \mathrm{~Hz}, 1 \mathrm{H}), 7.93-7.88(\mathrm{~m}, 2 \mathrm{H}), 7.84(\mathrm{dd}, J=8.1,1.6$ $\mathrm{Hz}, 1 \mathrm{H}), 7.52(\mathrm{~d}, J=8.7 \mathrm{~Hz}, 2 \mathrm{H}), 7.44(\mathrm{~d}, J=7.5 \mathrm{~Hz}, 2 \mathrm{H}), 7.38(\mathrm{t}, J$ $=7.4 \mathrm{~Hz}, 2 \mathrm{H}), 7.32(\mathrm{t}, J=7.2 \mathrm{~Hz}, 1 \mathrm{H}), 7.16(\mathrm{~d}, J=8.8 \mathrm{~Hz}, 2 \mathrm{H}), 5.14$ (s, 2H), $3.20(\mathrm{~s}, 3 \mathrm{H}), 2.67(\mathrm{~s}, 3 \mathrm{H}) ;{ }^{13} \mathrm{C}\left\{{ }^{1} \mathrm{H}\right\}$ NMR $(126 \mathrm{MHz}$, DMSO- $\left.d_{6} / \mathrm{D}_{2} \mathrm{O} / \mathrm{TFA}\right) \delta 160.3,151.0,146.9,141.7,141.5,139.5$, 137.6, 137.3, 133.6, 131.4, 131.0, 130.8, 130.2, 129.4, 129.0, 128.6, $125.3,122.9,121.6,116.5,70.3,44.1,21.5 ; \nu_{\max }\left(\mathrm{cm}^{-1}\right.$, thin film, ATR) $3041(w), 2921(w), 1732(w), 1605(s), 1513(m), 1488(w)$, $1469(\mathrm{w}), 1445(\mathrm{w}), 1303(\mathrm{~m}), 1289(\mathrm{~m}), 1243(\mathrm{~m}), 1151(\mathrm{~s}), 1143$ (s), $1072(\mathrm{w}), 974(\mathrm{~m}), 831(\mathrm{~s}), 808(\mathrm{w}), 767(\mathrm{~s}), 742(\mathrm{~m})$; HRMS (ESI+/TOF) $m / z\left[\mathrm{M}+\mathrm{H}^{+}\right.$calcd for $\mathrm{C}_{29} \mathrm{H}_{26} \mathrm{~N}_{3} \mathrm{O}_{3} \mathrm{~S} 496.1695$ 466.1589, found 496.1688; mp 245.0-248.5 ${ }^{\circ} \mathrm{C}$ ( $\left.\mathrm{MeOH} / \mathrm{DCM}\right)$, turned brown upon heating

4-(4-(4-(Methoxymethoxy) phenyl)-2-(2-methyl-4(methylsulfonyl)phenyl)-1H-imidazol-5-yl)pyridine (48). The title 
compound was prepared according to general procedure $C$, using 44 (39.0 mg, $0.10 \mathrm{mmol}, 1.00$ equiv), 4-(methoxymethoxy)phenyl boronic acid (23.0 mg, $0.125 \mathrm{mmol}, 1.25$ equiv), $\mathrm{Pd}(\mathrm{OAc})_{2}(2.3$ $\mathrm{mg}, 10 \mathrm{~mol} \%)$, and Aphos (6.7 mg, $24 \mathrm{~mol} \%)$. Purification by silica gel chromatography, eluting with EtOH in $\mathrm{CHCl}_{3}(21 \mathrm{~cm} \times 10 \mathrm{~mm}$, gradient elution, $0 \% \rightarrow 6 \%, 0.5 \%$ increases, $20.0 \mathrm{~mL}$ runs, $3-4 \mathrm{~mL}$ fractions) followed by repurification in silica gel using $\mathrm{MeOH}$ in DCM eluent $(21 \mathrm{~cm} \times 10 \mathrm{~mm}$, gradient elution, $0 \% \rightarrow 6 \%, 0.5 \%$ increases, $20 \mathrm{~mL}$ runs, $7 \mathrm{~mL}$ fractions), yielded 48 as a white solid (73\% yield, $33.0 \mathrm{mg}, 0.07 \mathrm{mmol}):{ }^{1} \mathrm{H}$ NMR $\left(500 \mathrm{MHz}\right.$, DMSO- $d_{6} /$ $\left.\mathrm{D}_{2} \mathrm{O}\right) \delta 8.38(\mathrm{~d}, J=4.7 \mathrm{~Hz}, 2 \mathrm{H}), 7.89-7.83(\mathrm{~m}, 2 \mathrm{H}), 7.80(\mathrm{~d}, J=$ $8.53,1 \mathrm{H}), 7.50(\mathrm{~d}, J=4.7 \mathrm{~Hz}, 2 \mathrm{H}), 7.42(\mathrm{~d}, J=8.53,2 \mathrm{H}) 7.10(\mathrm{~d}, J=$ $8.15 \mathrm{~Hz}, 2 \mathrm{H}), 5.20(\mathrm{~s}, 2 \mathrm{H}), 3.37(\mathrm{~s}, 3 \mathrm{H}), 3.19(\mathrm{~s}, 3 \mathrm{H}), 2.66(\mathrm{~s}, 3 \mathrm{H})$; ${ }^{13} \mathrm{C}\left\{{ }^{1} \mathrm{H}\right\}$ NMR $\left(126 \mathrm{MHz}, \mathrm{DMSO}-d_{6} / \mathrm{D}_{2} \mathrm{O}\right) \delta 157.8,150.0,145.7$, 143.2, 140.6, 139.0, 134.9, 134.4, 131.9, 130.9, 130.5, 130.1, 125.1, $123.8,121.8,117.4,94.5,56.6,44.2,21.6$ (note that extra signals in the ${ }^{13} \mathrm{C}\left\{{ }^{1} \mathrm{H}\right\}$ NMR spectra are due to the presence of tautomers); $\nu_{\max }$ $\left(\mathrm{cm}^{-1}\right.$, thin film, ATR) $2925(\mathrm{w}), 1600(\mathrm{~s}), 1513(\mathrm{~m}), 1491(\mathrm{w})$, $1444(\mathrm{w}), 1309(\mathrm{~m}), 1238(\mathrm{~m}), 1214(\mathrm{w}), 1200(\mathrm{w}), 1143(\mathrm{~s}), 1108$ (m), $1000(\mathrm{~m}), 970(\mathrm{~s}), 955(\mathrm{~m}), 918(\mathrm{w}), 834(\mathrm{~s}), 761(\mathrm{~s}), 741(\mathrm{~s})$; HRMS (ESI+/TOF) $m / z[\mathrm{M}+\mathrm{H}]^{+}$calcd for $\mathrm{C}_{24} \mathrm{H}_{24} \mathrm{~N}_{3} \mathrm{O}_{4} \mathrm{~S}$ 450.1487, found 450.1467; $\mathrm{mp} 225.0-226.4{ }^{\circ} \mathrm{C}(\mathrm{MeOH} / \mathrm{DCM})$.

3-(2-(2-Methyl-4-(methylsulfonyl)phenyl)-5-(pyridin-4-yl)- $1 \mathrm{H}$ imidazol-4-yl)benzenesulfonamide (49). The title compound was prepared according to general procedure C, using $44(39.0 \mathrm{mg}, 0.10$ mmol, 1.00 equiv), (3-aminosulfonylphenyl)boronic acid $(26.0 \mathrm{mg}$, $0.125 \mathrm{mmol}, 1.25$ equiv), $\mathrm{Pd}(\mathrm{OAc})_{2}(2.3 \mathrm{mg}, 10 \mathrm{~mol} \%)$, and Aphos (6.7 $\mathrm{mg}, 24 \mathrm{~mol} \%)$. Purification by preparative TLC, eluting with EtOH in $\mathrm{CHCl}_{3}\left(20 \mathrm{~cm} \times 20 \mathrm{~cm}\right.$ plate, $10 \% \mathrm{EtOH} / \mathrm{CHCl}_{3}$, two runs), yielded 49 as a white solid (62\% yield, $29.0 \mathrm{mg}, 0.06 \mathrm{mmol}$ ): ${ }^{1} \mathrm{H}$ NMR (500 MHz, DMSO- $\left.d_{6} / \mathrm{D}_{2} \mathrm{O} / \mathrm{TFA}\right) \delta 8.66(\mathrm{~d}, J=6.2 \mathrm{~Hz}$, $2 \mathrm{H}), 8.06-8.01(\mathrm{~m}, 3 \mathrm{H}), 7.99-7.90(\mathrm{~m}, 3 \mathrm{H}), 7.89-7.84(\mathrm{~m}, 2 \mathrm{H})$, $7.75(\mathrm{t}, J=7.7,1 \mathrm{H}), 3.23(\mathrm{~s}, 3 \mathrm{H}), 2.73(\mathrm{~s}, 3 \mathrm{H}) ;{ }^{13} \mathrm{C}\left\{{ }^{1} \mathrm{H}\right\}$ NMR $(126$ $\left.\mathrm{MHz}, \mathrm{DMSO}-d_{6} / \mathrm{D}_{2} \mathrm{O} / \mathrm{TFA}\right) \delta 150.5,147.5,145.2,141.8,141.3$, $139.1,136.0,133.6,133.0,131.8,130.8,130.6,130.4,130.0,127.1$, $126.5,125.0,123.0,43.9,21.5 ; \nu_{\max }\left(\mathrm{cm}^{-1}\right.$, thin film, ATR) $3296(\mathrm{br})$, $2931(\mathrm{w}), 1606(\mathrm{~m}), 1479(\mathrm{w}), 1410(\mathrm{w}), 1342(\mathrm{~m}), 1303(\mathrm{~m}), 1205$ $(\mathrm{w}), 1161(\mathrm{~s}), 1156(\mathrm{~s}), 1118(\mathrm{w}), 1079(\mathrm{w}), 1108(\mathrm{w}), 976(\mathrm{w}), 859$ $(\mathrm{w}), 833(\mathrm{~m}), 806(\mathrm{w}), 764(\mathrm{~m}), 746(\mathrm{~m}), 690(\mathrm{~s})$; HRMS (ESI + TOF) $m / z[\mathrm{M}+\mathrm{H}]^{+}$calcd for $\mathrm{C}_{22} \mathrm{H}_{21} \mathrm{~N}_{4} \mathrm{O}_{4} \mathrm{~S}_{2} 469.1004$, found 469.0997; mp 234.0-236.2 ${ }^{\circ} \mathrm{C}\left(\mathrm{EtOH} / \mathrm{CHCl}_{3}\right)$.

$\mathrm{N}$-Cyclopropyl-3-(2-(2-methyl-4-(methylsulfonyl)phenyl)-5-(pyridin-4-yl)-1H-imidazol-4-yl)benzenesulfonamide (50). The title compound was prepared according to general procedure $\mathrm{C}$, using 44 (39.0 mg, $0.10 \mathrm{mmol}, 1.00$ equiv), 3-(cyclopropylsulfamoyl)phenylboronic acid (31.0 mg, $0.125 \mathrm{mmol}, 1.25$ equiv), $\mathrm{Pd}(\mathrm{OAc})_{2}$ ( $2.3 \mathrm{mg}, 10 \mathrm{~mol} \%)$, and Aphos (6.7 mg, $24 \mathrm{~mol} \%)$. Purification by silica gel chromatography, eluting with EtOH in $\mathrm{CHCl}_{3}(21 \mathrm{~cm} \times 10$ $\mathrm{mm}$, gradient elution, $3 \% \rightarrow 8 \%, 0.5 \%$ increases, $20 \mathrm{~mL}$ runs, $3-4 \mathrm{~mL}$ fractions), yielded $\mathbf{5 0}$ as a white solid (57\% yield, $29.0 \mathrm{mg}, 0.06$ mmol): ${ }^{1} \mathrm{H}$ NMR (500 MHz, DMSO- $\left.d_{6} / \mathrm{D}_{2} \mathrm{O} / \mathrm{TFA}\right) \delta 8.63$ (d, $J=$ $6.8 \mathrm{~Hz}, 2 \mathrm{H}), 8.02(\mathrm{~d}, J=6.8 \mathrm{~Hz}, 2 \mathrm{H}), 7.97-7.84(\mathrm{~m}, 6 \mathrm{H}), 7.79(\mathrm{t}, J=$ $7.71 \mathrm{H}), 3.21(\mathrm{~s}, 3 \mathrm{H}), 2.70(\mathrm{~s}, 3 \mathrm{H}) .2 .15-2.09(\mathrm{~m}, 1 \mathrm{H}), 0.51-0.45$ (m, 2H), 0.41-0.36 (m, 2H); ${ }^{13} \mathrm{C}\left\{{ }^{1} \mathrm{H}\right\}$ NMR $\left(126 \mathrm{MHz}\right.$, DMSO-d $d_{6} /$ $\left.\mathrm{D}_{2} \mathrm{O} / \mathrm{TFA}\right) \delta 150.6,147.7,141.9,141.7,141.5,139.4,136.1,133.8$, $133.7,131.9,131.3,130.9,130.8,130.1,128.6,127.7,125.2,123.4$, 44.1, 24.7, 21.5, 5.9; $\nu_{\max }\left(\mathrm{cm}^{-1}\right.$, thin film, ATR) $3077(\mathrm{br}), 2925(\mathrm{w})$, $2835(\mathrm{w}), 1608(\mathrm{~m}), 1539(\mathrm{w}), 1475(\mathrm{w}), 1413(\mathrm{w}), 1334(\mathrm{~m}), 1318$ $(\mathrm{m}), 1222(\mathrm{w}), 1161(\mathrm{~s}), 1119(\mathrm{w}), 1103(\mathrm{w}), 1030(\mathrm{w}), 1008(\mathrm{w})$, $961(\mathrm{~m}), 890(\mathrm{w}), 836(\mathrm{~m}), 765(\mathrm{w}), 695(\mathrm{~m})$; HRMS (ESI+/TOF) $m / z[\mathrm{M}+\mathrm{H}]^{+}$calcd for $\mathrm{C}_{25} \mathrm{H}_{25} \mathrm{~N}_{4} \mathrm{O}_{4} \mathrm{~S}_{2}$ 509.1317, found 509.1317; $\mathrm{mp} 212.7-215.7{ }^{\circ} \mathrm{C}\left(\mathrm{EtOH} / \mathrm{CHCl}_{3}\right)$.

1-Methyl-5-(2-(2-methyl-4-(methylsulfonyl)phenyl)-5-(pyridin-4yl)-1H-imidazol-4-yl)-1H-indole (51). The title compound was prepared according to general procedure C, using $44(39.0 \mathrm{mg}$, $0.10 \mathrm{mmol}, 1.00$ equiv), $\mathrm{N}$-methylindole-5-boronic acid $(22.0 \mathrm{mg}$, $0.125 \mathrm{mmol}, 1.25$ equiv), $\mathrm{Pd}(\mathrm{OAc})_{2}(2.3 \mathrm{mg}, 10 \mathrm{~mol} \%)$, and Aphos $(6.7 \mathrm{mg}, 24 \mathrm{~mol} \%)$. Purification by silica gel chromatography, eluting with $\mathrm{EtOH}$ in $\mathrm{CHCl}_{3}(21 \mathrm{~cm} \times 10 \mathrm{~mm}$, gradient elution, $0 \% \rightarrow 6 \%$, $0.5 \%$ increases, $20 \mathrm{~mL}$ runs, $3-4 \mathrm{~mL}$ fractions) followed by repurification in silica gel using $\mathrm{MeOH}$ in DCM eluent $(21 \mathrm{~cm} \times$ $10 \mathrm{~mm}$, gradient elution, $0 \% \rightarrow 6 \%, 0.5 \%$ increases, $20 \mathrm{~mL}$ runs, $7 \mathrm{~mL}$ fractions), yielded 41 as a white solid (56\% yield, $25.0 \mathrm{mg}, 0.06$ mmol): $R_{f}=0.45$ (7\% EtOH/ $\mathrm{CHCl}_{3}$, Dragendorff stain); ${ }^{1} \mathrm{H}$ NMR $\left(400 \mathrm{MHz}, \mathrm{DMSO}-d_{6} / \mathrm{D}_{2} \mathrm{O} / \mathrm{TFA}\right) \delta 8.56(\mathrm{~d}, J=7.0 \mathrm{~Hz}, 2 \mathrm{H}), 8.04$ $(\mathrm{d}, J=7.0 \mathrm{~Hz}, 2 \mathrm{H}), 7.97(\mathrm{~d}, J=8.2 \mathrm{~Hz}, 1 \mathrm{H}), 7.92(\mathrm{~d}, J=1.4 \mathrm{~Hz}, 1 \mathrm{H})$, $7.86(\mathrm{dd}, J=1.6,8.2 \mathrm{~Hz}, 1 \mathrm{H}), 7.83(\mathrm{~d}, J=1.2 \mathrm{~Hz}, 1 \mathrm{H}), 7.60(\mathrm{~d}, J=$ $8.5 \mathrm{~Hz}, 1 \mathrm{H}), 7.41(\mathrm{~d}, J=3.0 \mathrm{~Hz}, 1 \mathrm{H}), 7.34(\mathrm{dd}, J=8.5,1.5 \mathrm{~Hz}, 1 \mathrm{H})$, $6.54(\mathrm{~d}, J=3.0 \mathrm{~Hz}, 1 \mathrm{H}), 3.82(\mathrm{~s}, 3 \mathrm{H}), 3.22(\mathrm{~s}, 1 \mathrm{H}), 2.73(\mathrm{~s}, 3 \mathrm{H})$ (note that the signal at $\delta 8.09 \mathrm{ppm}$ corresponds to residual $\mathrm{CHCl}_{3}$ in the sample); ${ }^{13} \mathrm{C}\left\{{ }^{1} \mathrm{H}\right\}$ NMR (126 MHz, DMSO- $\left.d_{6} / \mathrm{D}_{2} \mathrm{O} / \mathrm{TFA}\right) \delta$ $151.1,146.5,141.5,141.3,139.5,139.2,137.5,133.5,131.8,130.8$, $130.6,130.1,129.0,125.1,122.5,122.4,122.0,119.6,111.4,44.0$, $33.2,21.5$ (note that signal at $\delta 79.5 \mathrm{ppm}$ corresponds to residual $\mathrm{CHCl}_{3}$ in the sample and one carbon signal missing in the spectra); $\nu_{\max }\left(\mathrm{cm}^{-1}\right.$, thin film, ATR) 2914 (w), 2683 (br), 1603 (s), 1507 (w), $1485(w), 1441(w), 1430(w), 1378(w), 1309(s), 1286(w), 1243$ $(\mathrm{w}), 1210(\mathrm{w}), 1154(\mathrm{~s}), 1112(\mathrm{~m}), 1090(\mathrm{~m}), 1071(\mathrm{w}), 1003(\mathrm{w})$, $964(\mathrm{~m}), 951(\mathrm{~m}), 893(\mathrm{w}), 832(\mathrm{~s}), 815(\mathrm{w}), 763(\mathrm{~m}), 741(\mathrm{~m}), 730$ (m), $701(\mathrm{w})$; HRMS (ESI+/TOF) $m / z[\mathrm{M}+\mathrm{H}]^{+}$calcd for $\mathrm{C}_{25} \mathrm{H}_{23} \mathrm{~N}_{4} \mathrm{O}_{2} \mathrm{~S} 443.1542$, found 443.1529; mp $294.0{ }^{\circ} \mathrm{C}$ (dec).

4-(4-(Benzofuran-5-yl)-2-(2-methyl-4-(methylsulfonyl)phenyl)$1 \mathrm{H}$-imidazol-5-yl)pyridine (52). The title compound was prepared according to general procedure $\mathrm{C}$, using $44(39.0 \mathrm{mg}, 0.10 \mathrm{mmol}, 1.00$ equiv), benzofuran-5-boronic acid ( $21.0 \mathrm{mg}, 0.125 \mathrm{mmol}, 1.25$ equiv), $\mathrm{Pd}(\mathrm{OAc})_{2}(2.3 \mathrm{mg}, 10 \mathrm{~mol} \%)$, and Aphos (6.7 mg, $\left.24 \mathrm{~mol} \%\right)$. Purification by silica gel chromatography, eluting with $\mathrm{EtOH}$ in $\mathrm{CHCl}_{3}(21 \mathrm{~cm} \times 10 \mathrm{~mm}$, gradient elution, $0 \% \rightarrow 6 \%, 0.5 \%$ increases, $20 \mathrm{~mL}$ runs, $3-4 \mathrm{~mL}$ fractions) followed by repurification in silica gel using $\mathrm{MeOH}$ in $\mathrm{DCM}$ eluent $(21 \mathrm{~cm} \times 10 \mathrm{~mm}$, gradient elution, $0 \%$ $\rightarrow 6 \%, 0.5 \%$ increases, $20 \mathrm{~mL}$ runs, $7 \mathrm{~mL}$ fractions), yielded 52 as a white solid (59\% yield, $26.0 \mathrm{mg}, 0.06 \mathrm{mmol}): R_{f}=0.45(7 \% \mathrm{EtOH} /$ $\mathrm{CHCl}_{3}$, Dragendorff stain); ${ }^{1} \mathrm{H}$ NMR (500 MHz, DMSO- $d_{6} / \mathrm{D}_{2} \mathrm{O} /$ TFA) $\delta 8.57(\mathrm{~d}, J=6.9 \mathrm{~Hz}, 1 \mathrm{H}), 8.04-8.00(\mathrm{~m}, 3 \mathrm{H}), 7.95(\mathrm{~d}, J=8.2$ $\mathrm{Hz}, 1 \mathrm{H}), 7.92(\mathrm{~d}, J=8.5 \mathrm{~Hz}, 1 \mathrm{H}), 7.86(\mathrm{dd}, J=8.5,1.4 \mathrm{~Hz}, 1 \mathrm{H}), 7.76$ $(\mathrm{d}, J=8.5 \mathrm{~Hz}, 1 \mathrm{H}), 7.52(\mathrm{dd}, J=8.4,1.8 \mathrm{~Hz}, 1 \mathrm{H}), 7.03(\mathrm{~d}, J=1.4 \mathrm{~Hz}$, $1 \mathrm{H}), 3.21(\mathrm{~s}, 3 \mathrm{H}), 2.72(\mathrm{~s}, 3 \mathrm{H})$ (note that the signal at $\delta 8.09 \mathrm{ppm}$ corresponds to residual $\mathrm{CHCl}_{3}$ in the sample); ${ }^{13} \mathrm{C}\left\{{ }^{1} \mathrm{H}\right\}$ NMR $(126$ $\left.\mathrm{MHz}, \mathrm{DMSO}-d_{6} / \mathrm{D}_{2} \mathrm{O} / \mathrm{TFA}\right) \delta 155.6,151.0,147.9,146.9,141.6$, $141.4,139.4,138.0,133.7,131.4,130.7,130.1,128.9,126.2$, 125.2, $124.3,123.1,122.8,113.1,107.8,44.1,21.6$ (note that the signal at $\delta$ $79.5 \mathrm{ppm}$ corresponds to residual $\mathrm{CHCl}_{3}$ in the sample); $\nu_{\max }\left(\mathrm{cm}^{-1}\right.$, thin film, ATR) 2925 (w), 1601 (s), 1457 (w), 1444 (w), 1307 (m), $1210(\mathrm{w}), 1196(\mathrm{w}), 1150(\mathrm{~s}), 1107(\mathrm{~m}), 1086(\mathrm{w}), 1070(\mathrm{w}), 956$ (m), 869 (w), 833 (m), 763 (s), 743 (s); HRMS (ESI+/TOF) m/z $[\mathrm{M}+\mathrm{H}]^{+}$calcd for $\mathrm{C}_{24} \mathrm{H}_{20} \mathrm{~N}_{3} \mathrm{O}_{3} \mathrm{~S}$ 430.1225, found 430.1207; mp 232.0-233.4 ${ }^{\circ} \mathrm{C}(\mathrm{MeOH} / \mathrm{DCM})$.

4-(4-(Benzo[b]thiophen-5-yl)-2-(2-methyl-4-(methylsulfonyl)phenyl)-1H-imidazol-5-yl)pyridine (53). The title compound was prepared according to general procedure $\mathrm{C}$, using $44(39.0 \mathrm{mg}, 0.10$ mmol, 1.00 equiv), 2-(benzo[b]thiophen-5-yl)-4,4,5,5-tetramethyl1,3,2-dioxaborolane ${ }^{39}$ (S6) (33.0 mg, $0.125 \mathrm{mmol}, 1.25$ equiv), $\mathrm{Pd}(\mathrm{OAc})_{2}(2.3 \mathrm{mg}, 10 \mathrm{~mol} \%)$, and Aphos (6.7 mg, $\left.24 \mathrm{~mol} \%\right)$. Purification by silica gel chromatography, eluting with $\mathrm{EtOH}$ in $\mathrm{CHCl}_{3}(21 \mathrm{~cm} \times 10 \mathrm{~mm}$, gradient elution, $0 \% \rightarrow 6 \%, 0.5 \%$ increases, $20 \mathrm{~mL}$ runs, $3-4 \mathrm{~mL}$ fractions) followed by repurification in silica gel using $\mathrm{MeOH}$ in $\mathrm{DCM}$ eluent $(21 \mathrm{~cm} \times 10 \mathrm{~mm}$, gradient elution, $0 \%$ $\rightarrow 6 \%, 0.5 \%$ increases, $20 \mathrm{~mL}$ runs, $7 \mathrm{~mL}$ fractions), yielded 53 as a yellow solid (34\% yield, $15.0 \mathrm{mg}, 0.03 \mathrm{mmol}): R_{f}=0.45(7 \% \mathrm{EtOH} /$ $\mathrm{CHCl}_{3}$, Dragendorff stain); ${ }^{1} \mathrm{H}$ NMR $\left(500 \mathrm{MHz}, \mathrm{DMSO}-d_{6} / \mathrm{D}_{2} \mathrm{O} /\right.$ TFA) $\delta 8.56(\mathrm{~d}, J=6.9 \mathrm{~Hz}, 2 \mathrm{H}), 8.16-8.12(\mathrm{~m}, 2 \mathrm{H}), 8.04(\mathrm{~d}, J=6.9$ $\mathrm{Hz}, 2 \mathrm{H}), 7.95(\mathrm{~d}, J=8.2 \mathrm{~Hz}, 1 \mathrm{H}), 7.85(\mathrm{dd}, J=8.1,1.6 \mathrm{~Hz}, 1 \mathrm{H}) 7.81$ $(\mathrm{d}, J=5.4 \mathrm{~Hz}, 1 \mathrm{H}), 7.54(\mathrm{dd}, J=8.5,1.6 \mathrm{~Hz}, 1 \mathrm{H}), 7.52(\mathrm{~d}, J=5.4 \mathrm{~Hz}$, $1 \mathrm{H}), 3.21(\mathrm{~s}, 3 \mathrm{H}), 2.71(\mathrm{~s}, 3 \mathrm{H}) ;{ }^{13} \mathrm{C}\left\{{ }^{1} \mathrm{H}\right\}$ NMR $(126 \mathrm{MHz}$, DMSO$\left.d_{6} / \mathrm{D}_{2} \mathrm{O} / \mathrm{TFA}\right) \delta 151.1,147.2,141.7,141.4,141.3,140.8,139.5$, $137.9,133.8,131.5,130.8,130.2,129.9,125.6,125.5,125.3,125.1$, 125.0, 124.5, 123.0, 44.1, 21.6; $\nu_{\max }\left(\mathrm{cm}^{-1}\right.$, thin film, ATR) $2919(\mathrm{w})$, $2853(\mathrm{w}), 1602(\mathrm{~s}), 1488(\mathrm{w}), 1434(\mathrm{~m}), 1427(\mathrm{w}), 1304(\mathrm{~s}), 1213$ $(\mathrm{w}), 1201(\mathrm{w}), 1142(\mathrm{~s}), 1103(\mathrm{~m}), 1072(\mathrm{w}), 1049(\mathrm{w}), 992(\mathrm{w})$, $975(\mathrm{~m}), 955$ (m), $835(\mathrm{~m}), 816(\mathrm{~m}), 766$ (s); HRMS (ESI+/TOF) 
$m / z[\mathrm{M}+\mathrm{H}]^{+}$calcd for $\mathrm{C}_{24} \mathrm{H}_{20} \mathrm{~N}_{3} \mathrm{O}_{2} \mathrm{~S}_{2}$ 446.0991, found 446.0985; mp $274.0^{\circ} \mathrm{C}(\mathrm{dec})$.

4-(2-(2-Methyl-4-(methylsulfonyl)phenyl)-5-(pyridin--4-yl)-1Himidazol-4-yl)quinoline (54). The title compound was prepared according to general procedure C, using $44(39.0 \mathrm{mg}, 0.10 \mathrm{mmol}, 1.00$ equiv), quinoline-6-boronic acid $(22.0 \mathrm{mg}, 0.125 \mathrm{mmol}, 1.25$ equiv), $\mathrm{Pd}(\mathrm{OAc})_{2}(2.3 \mathrm{mg}, 10 \mathrm{~mol} \%)$, and Aphos $(6.7 \mathrm{mg}, 24 \mathrm{~mol} \%)$. Purification by silica gel chromatography, eluting with $\mathrm{EtOH}$ in $\mathrm{CHCl}_{3}(21 \mathrm{~cm} \times 10 \mathrm{~mm}$, gradient elution, $0 \% \rightarrow 8 \%, 0.5 \%$ increases, $20 \mathrm{~mL}$ runs, $3-4 \mathrm{~mL}$ fractions) followed by repurification in silica gel using $\mathrm{MeOH}$ in DCM eluent $(21 \mathrm{~cm} \times 10 \mathrm{~mm}$, gradient elution, $0 \%$ $\rightarrow 8 \%, 0.5 \%$ increases, $20 \mathrm{~mL}$ runs, $7 \mathrm{~mL}$ fractions), yielded 54 as a white solid ( $52 \%$ yield, $23.0 \mathrm{mg}, 0.05 \mathrm{mmol}): R_{f}=0.42(7 \% \mathrm{EtOH} /$ $\mathrm{CHCl}_{3}$, Dragendorff stain); ${ }^{1} \mathrm{H}$ NMR $\left(500 \mathrm{MHz}\right.$, DMSO- $d_{6} / \mathrm{D}_{2} \mathrm{O} /$ TFA) $\delta 9.22(\mathrm{~d}, J=5.1 \mathrm{~Hz}, 1 \mathrm{H}), 9.12(\mathrm{~d}, J=8.3 \mathrm{~Hz}, 1 \mathrm{H}), 8.62-8.56$ $(\mathrm{m}, 3 \mathrm{H}), 8.33(\mathrm{~d}, J=8.8 \mathrm{~Hz}, 1 \mathrm{H}), 8.27(\mathrm{dd}, J=8.9,1.4 \mathrm{~Hz}, 1 \mathrm{H})$, $8.11-8.05(\mathrm{~m}, 3 \mathrm{H}), 7.96(\mathrm{~d}, J=8.2 \mathrm{~Hz}, 1 \mathrm{H}), 7.92(\mathrm{~s}, 1 \mathrm{H}), 7.87(\mathrm{~d}, J$ $=8.1 \mathrm{~Hz}, 1 \mathrm{H}), 3.21(\mathrm{~s}, 3 \mathrm{H}), 2.72(\mathrm{~s}, 3 \mathrm{H}) ;{ }^{13} \mathrm{C}\left\{{ }^{1} \mathrm{H}\right\}$ NMR $(126 \mathrm{MHz}$, DMSO-d $\left.d_{6} / \mathrm{D}_{2} \mathrm{O} / \mathrm{TFA}\right) \delta 150.4,148.3,147.2,146.9,142.0,141.6$, $139.6,139.1,135.8,135.3,133.9,132.4,131.3,130.9,130.3,130.0$, $129.6,125.3,123.8,123.6,123.4,44.2,21.6 ; \nu_{\max }\left(\mathrm{cm}^{-1}\right.$, thin film, ATR) $1729(\mathrm{w}), 1598(\mathrm{~m}), 1510(\mathrm{w}), 1490(\mathrm{w}), 1304(\mathrm{~m}), 1141(\mathrm{~s})$, $1103(\mathrm{w}), 1073(\mathrm{w}), 954(\mathrm{~m}), 883(\mathrm{w}), 836(\mathrm{~m}), 765(\mathrm{~m}), 743(\mathrm{w})$; HRMS (ESI+/TOF) $m / z[\mathrm{M}+\mathrm{H}]^{+}$calcd for $\mathrm{C}_{25} \mathrm{H}_{21} \mathrm{~N}_{4} \mathrm{O}_{2} \mathrm{~S} 441.1385$, found 441.1372 ; mp $225.0-227.0{ }^{\circ} \mathrm{C}(\mathrm{dec})$, turned brown at 160.0 ${ }^{\circ} \mathrm{C}$.

6-(2-(2-Methyl-4-(methylsulfonyl)phenyl)-4-(naphthalen-2-yl)$1 \mathrm{H}$-imidazol-5-yl)pyridine (55). The title compound was prepared according to general procedure C, using $44(39.0 \mathrm{mg}, 0.10 \mathrm{mmol}, 1.00$ equiv), 2-naphthaleneboronic acid $(22.0 \mathrm{mg}, 0.125 \mathrm{mmol}, 1.25$ equiv), $\mathrm{Pd}(\mathrm{OAc})_{2}(2.3 \mathrm{mg}, 10 \mathrm{~mol} \%)$, and Aphos $(6.7 \mathrm{mg}, 24 \mathrm{~mol}$ $\%)$. Purification by silica gel chromatography, eluting with EtOH in $\mathrm{CHCl}_{3}(21 \mathrm{~cm} \times 10 \mathrm{~mm}$, gradient elution, $0 \% \rightarrow 6 \%, 0.5 \%$ increases, $20 \mathrm{~mL}$ runs, $3-4 \mathrm{~mL}$ fractions) followed by repurification in silica gel using $\mathrm{MeOH}$ in DCM eluent $(21 \mathrm{~cm} \times 10 \mathrm{~mm}$, gradient elution, $0 \%$ $\rightarrow 6 \%, 0.5 \%$ increases, $20 \mathrm{~mL}$ runs, $7 \mathrm{~mL}$ fractions), yielded 55 as a white solid (69\% yield, $31.0 \mathrm{mg}, 0.07 \mathrm{mmol}): R_{f}=0.42(7 \% \mathrm{EtOH} /$ $\mathrm{CHCl}_{3}$, Dragendorff stain); ${ }^{1} \mathrm{H}$ NMR (500 MHz, DMSO- $d_{6} / \mathrm{D}_{2} \mathrm{O} /$ TFA) $\delta 8.59(\mathrm{~d}, J=6.9 \mathrm{~Hz}, 2 \mathrm{H}), 8.21(\mathrm{~s}, 1 \mathrm{H}), 8.09-8.04(\mathrm{~m}, 3 \mathrm{H})$, $8.02-7.95(\mathrm{~m}, 3 \mathrm{H}), 7.92(\mathrm{~s}, 1 \mathrm{H}), 7.87(\mathrm{dd}, J=8.1,1.2 \mathrm{~Hz}, 1 \mathrm{H}), 7.66$ $(\mathrm{dd}, J=8.4,1.6 \mathrm{~Hz}, 1 \mathrm{H}), 7.64-7.57(\mathrm{~m}, 2 \mathrm{H}), 3.22(\mathrm{~s}, 3 \mathrm{H}), 2.74(\mathrm{~s}$, $3 \mathrm{H}$ ) (note that the signal at $\delta 8.09 \mathrm{ppm}$ corresponds to residual $\mathrm{CHCl}_{3}$ in the sample); ${ }^{13} \mathrm{C}\left\{{ }^{1} \mathrm{H}\right\}$ NMR $\left(126 \mathrm{MHz}, \mathrm{DMSO}-d_{6} / \mathrm{D}_{2} \mathrm{O} /\right.$ TFA) $\delta 151.1,147.3,141.7,141.3,139.3,137.5,133.8,133.8,133.5$, $131.8,130.7,130.1,129.8,129.2,129.0,128.5,128.2,127.9,126.9$, $126.8,125.1,123.0,44.1,21.6$ (note that the signal at $\delta 79.5 \mathrm{ppm}$ corresponds to residual $\mathrm{CHCl}_{3}$ in the sample); HRMS (ESI+/TOF) $m / z[\mathrm{M}+\mathrm{H}]^{+}$calcd for $\mathrm{C}_{26} \mathrm{H}_{22} \mathrm{~N}_{3} \mathrm{O}_{2} \mathrm{~S} 440.1433$, found 440.1418 .

6-(2-(2-Methyl-4-(methylsulfonyl)phenyl)-5-(pyridin-4-yl)- $1 \mathrm{H}$ imidazol-4-yl)naphthalen-2-ol (56). The title compound was prepared according to general procedure C, using $44(39.0 \mathrm{mg}$, $0.10 \mathrm{mmol}, 1.00$ equiv), 6-(4,4,5,5-tetramethyl-1,3,2-dioxaborolan-2yl)naphthalen-2-ol (S7) (34.0 mg, $0.125 \mathrm{mmol}, 1.25$ equiv), $\mathrm{Pd}(\mathrm{OAc})_{2}(2.3 \mathrm{mg}, 10 \mathrm{~mol} \%)$, and Aphos (6.7 mg, $\left.24 \mathrm{~mol} \%\right)$. Purification by silica gel chromatography, eluting with $\mathrm{EtOH}$ in $\mathrm{CHCl}_{3}(21 \mathrm{~cm} \times 10 \mathrm{~mm}$, gradient elution, $4 \% \rightarrow 9 \%, 0.5 \%$ increases, $20 \mathrm{~mL}$ runs, $3-4 \mathrm{~mL}$ fractions) followed by repurification in silica gel using $\mathrm{MeOH}$ in DCM eluent $(21 \mathrm{~cm} \times 10 \mathrm{~mm}$, gradient elution, $4 \%$ $\rightarrow 9 \%, 0.5 \%$ increases, $20 \mathrm{~mL}$ runs, $7 \mathrm{~mL}$ fractions), yielded 56 as a pale yellow solid $(67 \%$ yield, $30.0 \mathrm{mg}, 0.07 \mathrm{mmol}): R_{f}=0.28(7 \%$ EtOH/ $\mathrm{CHCl}_{3}$, Dragendorff stain); ${ }^{1} \mathrm{H}$ NMR $\left(500 \mathrm{MHz}\right.$, DMSO- $d_{6} /$ $\left.\mathrm{D}_{2} \mathrm{O} / \mathrm{TFA}\right) \delta 8.58(\mathrm{~d}, J=6.7 \mathrm{~Hz}, 2 \mathrm{H}), 8.10-8.04(\mathrm{~m}, 3 \mathrm{H}), 7.97(\mathrm{~d}, J$ $=8.1 \mathrm{~Hz}, 1 \mathrm{H}), 7.92(\mathrm{~s}, 1 \mathrm{H}), 7.88-7.81(\mathrm{~m}, 3 \mathrm{H}) 7.54(\mathrm{dd}, J=8.5,1.2$ $\mathrm{Hz}, 1 \mathrm{H}), 7.22(\mathrm{~d}, J=1.9 \mathrm{~Hz}, 1 \mathrm{H}), 7.16(\mathrm{dd}, J=8.8,2.2 \mathrm{~Hz}, 1 \mathrm{H}), 3.21$ (s, 3H), $2.72(\mathrm{~s}, 3 \mathrm{H}) ;{ }^{13} \mathrm{C}\left\{{ }^{1} \mathrm{H}\right\}$ NMR (126 MHz, DMSO- $d_{6} / \mathrm{D}_{2} \mathrm{O} /$ TFA) $\delta 157.0,151.1,147.1,141.6,141.4,139.4,138.0,135.7,133.7$, $131.4,131.0,130.7,130.2,129.2,128.3,128.0,127.1,125.2,123.5$, $122.8,120.3,109.5,44.1,21.6 ; \nu_{\max }\left(\mathrm{cm}^{-1}\right.$, thin film, ATR) $3221(\mathrm{br})$, $2927(\mathrm{w}), 2851(\mathrm{w}), 1626(\mathrm{w}), 1608(\mathrm{~s}), 1572(\mathrm{w}), 1436(\mathrm{w}), 1396$ $(\mathrm{w}), 1305(\mathrm{~s}), 1250(\mathrm{w}), 1211(\mathrm{~m}), 1163(\mathrm{w}), 1144(\mathrm{~s}), 1124(\mathrm{w})$, $1114(\mathrm{~m}), 1038(\mathrm{~m}), 1013(\mathrm{w}), 1001(\mathrm{w}), 947(\mathrm{~m}), 915(\mathrm{w}), 878(\mathrm{~s}$ $837(\mathrm{~m}), 829(\mathrm{~m}), 820(\mathrm{w}), 767$ (s); HRMS (ESI+/TOF) $\mathrm{m} / z[\mathrm{M}+$ $\mathrm{H}]^{+}$calcd for $\mathrm{C}_{26} \mathrm{H}_{22} \mathrm{~N}_{3} \mathrm{O}_{3} \mathrm{~S} 456.1382$, found 456.1358 ; mp $250.0{ }^{\circ} \mathrm{C}$ (dec).

4-(4-(6-Methoxynaphthalen-2-yl)-2-(2-methyl-4(methylsulfonyl)phenyl)-1H-imidazol-5-yl)pyridine (57). The title compound was prepared according to general procedure $\mathrm{C}$, using 44 (79.0 mg, $0.20 \mathrm{mmol}, 1.00$ equiv), 6-methoxy-2-naphthaleneboronic acid (53.0 mg, $0.25 \mathrm{mmol}, 1.25$ equiv), $\mathrm{Pd}(\mathrm{OAc})_{2}(4.6 \mathrm{mg}, 10 \mathrm{~mol}$ \%), Aphos (13.4 mg, $24 \mathrm{~mol} \%), \mathrm{K}_{2} \mathrm{CO}_{3}(83 \mathrm{mg}, 0.06 \mathrm{mmol}, 3.00$ equiv), degassed DME $(1.5 \mathrm{~mL})$, and distilled $\mathrm{H}_{2} \mathrm{O}(0.5 \mathrm{~mL})$. Purification by silica gel chromatography, eluting with EtOH in DCM $(21 \mathrm{~cm} \times 20 \mathrm{~mm}$, gradient elution, $0 \% \rightarrow 8 \%, 0.5 \%$ increases, $20 \mathrm{~mL}$ runs, $3-4 \mathrm{~mL}$ fractions), yielded $\mathbf{5 7}$ as a white solid (97\% yield, 91.0 $\mathrm{mg}, 0.19 \mathrm{mmol}): R_{f}=0.37$ (7\% EtOH/ $\mathrm{CHCl}_{3}$, Dragendorff stain); ${ }^{1} \mathrm{H}$ NMR (DMSO- $\left.d_{6} / \mathrm{D}_{2} \mathrm{O} / \mathrm{TFA}\right) \delta 8.58(\mathrm{~d}, J=7.0 \mathrm{~Hz}, 2 \mathrm{H}), 8.12(\mathrm{~s}$, $1 \mathrm{H}), 8.05(\mathrm{~d}, J=7.0 \mathrm{~Hz}, 2 \mathrm{H}), 7.98(\mathrm{~d}, J=1.7 \mathrm{~Hz}, 1 \mathrm{H}), 7.96(\mathrm{~d}, J=$ $2.2 \mathrm{~Hz}, 1 \mathrm{H}), 7.92(\mathrm{~s}, 1 \mathrm{H}), 7.89(\mathrm{~d}, J=9.0 \mathrm{~Hz}, 1 \mathrm{H}), 7.86(\mathrm{dd}, J=8.2$, $1.5 \mathrm{~Hz}, 1 \mathrm{H}), 7.60(\mathrm{dd}, J=8.5,1.5,1 \mathrm{H}), 7.40(\mathrm{~d}, J=2.3 \mathrm{~Hz}, 1 \mathrm{H}), 7.23$ $(\mathrm{dd}, J=9.0,2.5 \mathrm{~Hz}, 1 \mathrm{H}), 3.88(\mathrm{~s}, 3 \mathrm{H}), 3.22(\mathrm{~s}, 3 \mathrm{H}), 2.73(\mathrm{~s}, 3 \mathrm{H})$; ${ }^{13} \mathrm{C}\left\{{ }^{1} \mathrm{H}\right\}$ NMR $\left(126 \mathrm{MHz}\right.$, DMSO- $\left.d_{6} / \mathrm{D}_{2} \mathrm{O} / \mathrm{TFA}\right) \delta 159.1,151.1$, $147.1,141.6,141.3,139.3,137.8,135.4,133.7,131.6,130.7,130.6$, $130.1,129.0,129.0,128.7,127.2,125.1,124.4,122.8,120.3,106.8$, 56.1, 44.1, 21.6; $\nu_{\max }\left(\mathrm{cm}^{-1}\right.$, thin film, ATR) $3125(\mathrm{br}), 1629(\mathrm{w})$, $1600(\mathrm{~s}), 1498(\mathrm{w}), 1302(\mathrm{~s}), 1263(\mathrm{~m}), 1205$ (m), 1147 (s), 1110 $(\mathrm{m}), 1070(\mathrm{w}), 953(\mathrm{~m}), 859(\mathrm{~m}), 835(\mathrm{~m}), 767(\mathrm{~m}), 740(\mathrm{w})$; HRMS (ESI+/TOF) $m / z[\mathrm{M}+\mathrm{H}]^{+}$calcd for $\mathrm{C}_{27} \mathrm{H}_{24} \mathrm{~N}_{3} \mathrm{O}_{3} \mathrm{~S}$ 470.1538 , found $470.1551 ; \mathrm{mp} 256.0{ }^{\circ} \mathrm{C}$ ( dec), turned brown at $254.0^{\circ} \mathrm{C}$.

4-(4-(6-Ethoxynaphthalen-2-yl)-2-(2-methyl-4-(methylsulfonyl)phenyl)-1H-imidazol-5-yl)pyridine (58). The title compound was prepared according to general procedure $\mathrm{C}$, using $44(39.0 \mathrm{mg}, 0.10$ mmol, 1.00 equiv), 2-(6-ethoxynaphthalen-2-yl)-4,4,5,5-tetramethyl1,3,2-dioxaborolane (S9) (37.0 mg, $0.125 \mathrm{mmol}, 1.25$ equiv), $\mathrm{Pd}(\mathrm{OAc})_{2}(2.3 \mathrm{mg}, 10 \mathrm{~mol} \%)$, and Aphos (6.7 mg, $\left.24 \mathrm{~mol} \%\right)$. Purification by silica gel chromatography, eluting with EtOH in $\mathrm{CHCl}_{3}(21 \mathrm{~cm} \times 10 \mathrm{~mm}$, gradient elution, $0 \% \rightarrow 6 \%, 0.5 \%$ increases, $20 \mathrm{~mL}$ runs, $3-4 \mathrm{~mL}$ fractions) followed by repurification in silica gel using $\mathrm{MeOH}$ in DCM eluent $(16 \mathrm{~cm} \times 10 \mathrm{~mm}$, gradient elution, $0 \%$ $\rightarrow 6 \%, 0.5 \%$ increases, $20 \mathrm{~mL}$ runs, $7 \mathrm{~mL}$ fractions), yielded $\mathbf{5 8}$ as a white solid (64\% yield, $31.0 \mathrm{mg}, 0.06 \mathrm{mmol}): R_{f}=0.43(7 \% \mathrm{EtOH} /$ $\mathrm{CHCl}_{3}$, Dragendorff stain); ${ }^{1} \mathrm{H}$ NMR $\left(500 \mathrm{MHz}, \mathrm{DMSO}-d_{6} / \mathrm{D}_{2} \mathrm{O} /\right.$ TFA) $\delta 8.58(\mathrm{~d}, J=6.8 \mathrm{~Hz}, 2 \mathrm{H}), 8.11(\mathrm{~s}, 1 \mathrm{H}), 8.06(\mathrm{~d}, J=6.8 \mathrm{~Hz}$, $2 \mathrm{H}), 7.97(\mathrm{~d}, J=8.3 \mathrm{~Hz}, 1 \mathrm{H}), 7.95(\mathrm{~d}, J=8.6 \mathrm{~Hz}, 1 \mathrm{H}) 7.92(\mathrm{~s}, 1 \mathrm{H})$, $7.90-7.84(\mathrm{~m}, 2 \mathrm{H}), 7.59(\mathrm{dd}, J=8.5,1.4 \mathrm{~Hz}, 1 \mathrm{H}), 7.38(\mathrm{~d}, J=1.9 \mathrm{z}$, $1 \mathrm{H}), 7.21(\mathrm{dd}, J=8.9,2.3 \mathrm{~Hz}, 1 \mathrm{H}), 3.22(\mathrm{~s}, 3 \mathrm{H}), 2.73(\mathrm{~s}, 3 \mathrm{H}), 1.38$ $(\mathrm{t}, J=7.0 \mathrm{~Hz}, 3 \mathrm{H})$ (note that $\mathrm{CH}_{2}$ of the ethoxy group is not observed due to superposition of HOD signal); ${ }^{1} \mathrm{H}$ NMR $(500 \mathrm{MHz}$, DMSO- $\left.d_{6}\right) \delta 13.10(\mathrm{~s}, 1 \mathrm{H}), 8.45(\mathrm{~d}, J=4.8 \mathrm{~Hz}, 2 \mathrm{H}), 8.09(\mathrm{~s}, 1 \mathrm{H})$, $8.04(\mathrm{~d}, J=8.1 \mathrm{~Hz}, 1 \mathrm{H}), 7.95-7.85(\mathrm{~m}, 4 \mathrm{H}), 7.61-7.50(\mathrm{~m}, 3 \mathrm{H})$, $7.40(\mathrm{~d}, J=2.2 \mathrm{~Hz}, 1 \mathrm{H}), 7.23(\mathrm{dd}, J=8.9,2.5 \mathrm{~Hz}, 1 \mathrm{H}), 4.19(\mathrm{q}, J=$ $7.0 \mathrm{~Hz}, 2 \mathrm{H}), 3.28(\mathrm{~s}, 3 \mathrm{H}), 2.82(\mathrm{~s}, 3 \mathrm{H}), 1.43(\mathrm{t}, J=7.0 \mathrm{~Hz}, 3 \mathrm{H})$; ${ }^{13} \mathrm{C}\left\{{ }^{1} \mathrm{H}\right\}$ NMR (126 MHz, DMSO- $\left.d_{6} / \mathrm{D}_{2} \mathrm{O} / \mathrm{TFA}\right) \delta 158.3,151.1$, 147.1, 141.6, 141.3, 139.3, 137.8, 135.4, 133.7, 131.6, 130.68, 130.65, $130.1,129.0,128.9,128.6,127.2,125.1,124.3,122.8,120.6,107.4$, 64.2, 44.1, 21.6, $15.1 ;{ }^{13} \mathrm{C}\left\{{ }^{1} \mathrm{H}\right\}$ NMR (126 MHz, DMSO- $\left.d_{6} / \mathrm{D}_{2} \mathrm{O}\right) \delta$ $157.3,149.7,145.1,142.2,140.0,137.9,134.3,134.2,134.0,131.6$, $129.7,129.6,129.3,128.3,127.9,127.4,127.1,125.1,124.4,120.8$, $119.7,106.7,63.3,43.5,21.5,14.7 ; \nu_{\max }\left(\mathrm{cm}^{-1}\right.$, thin film, ATR) 3033 (br), $2928(\mathrm{w}), 1631(\mathrm{w}), 1600(\mathrm{~s}), 1497(\mathrm{w}), 1442(\mathrm{w}), 1400(\mathrm{w})$, 1319 (m), 1300 (m), 1261 (m), 1207 (w), 1144 (s), 1094 (m), 1041 (m), $994(\mathrm{~m}), 834(\mathrm{~m}), 768(\mathrm{~s}), 742(\mathrm{~s}), 700(\mathrm{w})$; HRMS (ESI $+/$ TOF) $m / z[\mathrm{M}+\mathrm{H}]^{+}$calcd for $\mathrm{C}_{28} \mathrm{H}_{26} \mathrm{~N}_{3} \mathrm{O}_{3} \mathrm{~S}$ 484.1695, found 484.1697; mp $250.0{ }^{\circ} \mathrm{C}$ (dec).

4-(4-(6-Cyclopropoxynaphthalen-2-yl)-2-(2-methyl-4(methylsulfonyl)phenyl)-1H-imidazol-5-yl)pyridine (59). The title compound was prepared according to general procedure $C$, using 44 (39.0 $\mathrm{mg}, 0.10 \mathrm{mmol}, 1.00$ equiv), 2-(6-cyclopropoxynaphthalen-2yl)-4,4,5,5-tetramethyl-1,3,2-dioxaborolane ${ }^{40}$ (S11) $(37.0 \mathrm{mg}, 0.125$ mmol, 1.25 equiv), $\mathrm{Pd}(\mathrm{OAc})_{2}(2.3 \mathrm{mg}, 10 \mathrm{~mol} \%)$, and Aphos (6.7 
$\mathrm{mg}, 24 \mathrm{~mol} \%$ ). Purification by silica gel chromatography, eluting with EtOH in $\mathrm{CHCl}_{3}(21 \mathrm{~cm} \times 10 \mathrm{~mm}$, gradient elution, $0 \% \rightarrow 6 \%, 0.5 \%$ increases, $20 \mathrm{~mL}$ runs, $3-4 \mathrm{~mL}$ fractions) followed by repurification in silica gel using $\mathrm{MeOH}$ in DCM eluent $(21 \mathrm{~cm} \times 10 \mathrm{~mm}$, gradient elution, $0 \% \rightarrow 6 \%, 0.5 \%$ increases, $20 \mathrm{~mL}$ runs, $7 \mathrm{~mL}$ fractions), yielded 59 as a white solid (63\% yield, $31.0 \mathrm{mg}, 0.06 \mathrm{mmol}$ ): $R_{f}=0.33$ (7\% EtOH/ $\mathrm{CHCl}_{3}$, Dragendorff stain); ${ }^{1} \mathrm{H}$ NMR (500 MHz, DMSO$\left.d_{6}\right) \delta 13.10(\mathrm{~s}, 1 \mathrm{H}), 8.65-8.39(\mathrm{~m}, 2 \mathrm{H}), 8.15-7.76(\mathrm{~m}, 6 \mathrm{H}), 7.68-$ $7.48(\mathrm{~m}, 4 \mathrm{H}), 7.87-7.81(\mathrm{~m}, 3 \mathrm{H}), 7.29-7.13(\mathrm{~m}, 1 \mathrm{H}), 4.00(\mathrm{~s}, 1 \mathrm{H})$, $3.28(\mathrm{~s}, 3 \mathrm{H}), 2.82(\mathrm{~s}, 3 \mathrm{H}), 0.92-0.85(\mathrm{~m}, 2 \mathrm{H}), 0.79-0.71(\mathrm{~m}, 2 \mathrm{H})$; ${ }^{1} \mathrm{H}$ NMR (600 MHz, DMSO- $\left.d_{6} / \mathrm{D}_{2} \mathrm{O} / \mathrm{TFA}\right) \delta 8.67(\mathrm{~d}, J=7.0 \mathrm{~Hz}$, $2 \mathrm{H}), 8.19(\mathrm{~s}, 1 \mathrm{H}), 8.07(\mathrm{~d}, J=7.0 \mathrm{~Hz}, 2 \mathrm{H}), 8.04(\mathrm{~d}, J=8.2 \mathrm{~Hz}, 1 \mathrm{H})$, $8.02(\mathrm{~d}, J=8.6 \mathrm{~Hz}, 1 \mathrm{H}), 7.96(\mathrm{~s}, 1 \mathrm{H}), 7.93(\mathrm{~d}, J=9.0 \mathrm{~Hz}, 1 \mathrm{H}), 7.90$ $(\mathrm{dd}, J=1.5,8.2 \mathrm{~Hz}, 1 \mathrm{H}), 7.68-7.65(\mathrm{~m}, 2 \mathrm{H}), 7.27(\mathrm{dd}, J=2.4,8.9$ $\mathrm{Hz}, 1 \mathrm{H}), 3.27(\mathrm{~s}, 3 \mathrm{H}), 2.80(\mathrm{~s}, 3 \mathrm{H}), 0.92-0.87(\mathrm{~m}, 2 \mathrm{H}), 0.75-0.72$ $(\mathrm{m}, 2 \mathrm{H})$ (note that the $\mathrm{CH}$ of the cyclopropoxy group is not observed due to superposition of HOD signal); ${ }^{13} \mathrm{C}\left\{{ }^{1} \mathrm{H}\right\}$ NMR $(126 \mathrm{MHz}$, DMSO- $\left.d_{6}\right) \delta 157.4,149.7,145.1,142.1,140.0,137.9,134.4,134.0$, $129.7,129.5,129.2,128.6,127.8,127.4,127.1,125.3,124.3,120.7$, $119.3,108.0,51.0,43.5,21.5,6.0$ (note that two carbon signals in the ${ }^{13} \mathrm{C}\left\{{ }^{1} \mathrm{H}\right\}$ NMR are missing); ${ }^{13} \mathrm{C}\left\{{ }^{1} \mathrm{H}\right\}$ NMR (151 MHz, DMSO- $d_{6} /$ $\left.\mathrm{D}_{2} \mathrm{O} / \mathrm{TFA}\right) \delta 158.0,150.9,146.7,141.4,140.9,138.6,137.4,134.8$, $133.4,131.6,130.2,129.9,129.8,128.8,128.7,128.2,127.0,124.7$, 124.4, 122.1, 119.8, 108.3, 51.4, 43.6, 21.5, 6.2; $\nu_{\max }\left(\mathrm{cm}^{-1}\right.$, thin film, ATR) $3038(\mathrm{w}), 2927(\mathrm{w}), 1629(\mathrm{w}), 1603(\mathrm{~s}), 1573(\mathrm{w}), 1494(\mathrm{w})$, $1445(\mathrm{w}), 1354(\mathrm{w}), 1304(\mathrm{~m}), 1260(\mathrm{~m}), 1216(\mathrm{~m}), 1149(\mathrm{~s}), 1120$ (w), $1107(\mathrm{~m}), 1074(\mathrm{w}), 996(\mathrm{w}), 986(\mathrm{~s}), 966(\mathrm{w}), 953(\mathrm{~m}), 872$ (w), $836(\mathrm{~s}), 804(\mathrm{w}), 764(\mathrm{~s}), 742(\mathrm{~m})$; HRMS (ESI+/TOF) $\mathrm{m} / \mathrm{z}$ $[\mathrm{M}+\mathrm{H}]^{+}$calcd for $\mathrm{C}_{29} \mathrm{H}_{26} \mathrm{~N}_{3} \mathrm{O}_{3} \mathrm{~S}$ 496.1695, found 496.1715; mp $268.0{ }^{\circ} \mathrm{C}$ (dec).

tert-Butyl (6-(2-(2-Methyl-4-(methylsulfonyl)phenyl)-5-(pyridin4-yl)-1H-imidazol-4-yl)naphthalen-2-yl)carbamate (60). The title compound was prepared according to general procedure $C$, using 44 (39.0 mg, $0.10 \mathrm{mmol}, 1.00$ equiv), tert-butyl (6-(4,4,5,5-tetramethyl1,3,2-dioxaborolan-2-yl)naphthalen-2-yl) carbamate $^{41}$ (S13) $(46.0 \mathrm{mg}$, $0.125 \mathrm{mmol}, 1.25$ equiv), $\mathrm{Pd}(\mathrm{OAc})_{2}(2.3 \mathrm{mg}, 10 \mathrm{~mol} \%)$, and Aphos $(6.7 \mathrm{mg}, 24 \mathrm{~mol} \%)$. Purification by silica gel chromatography, eluting with $\mathrm{EtOH}$ in $\mathrm{CHCl}_{3}(21 \mathrm{~cm} \times 10 \mathrm{~mm}$, gradient elution, $0 \% \rightarrow 6 \%$, $0.5 \%$ increases, $20 \mathrm{~mL}$ runs, $3-4 \mathrm{~mL}$ fractions) followed by repurification in silica gel using $\mathrm{MeOH}$ in DCM eluent $(21 \mathrm{~cm} \times$ $10 \mathrm{~mm}$, gradient elution, $0 \% \rightarrow 6 \%, 0.5 \%$ increases, $20 \mathrm{~mL}$ runs, $7 \mathrm{~mL}$ fractions), yielded 60 as a yellow solid ( $40 \%$ yield, $22.0 \mathrm{mg}, 0.04$ $\mathrm{mmol}): R_{f}=0.47$ (7\% EtOH/ $\mathrm{CHCl}_{3}$, Dragendorff stain); ${ }^{1} \mathrm{H}$ NMR $\left(500 \mathrm{MHz}, \mathrm{DMSO}-d_{6}\right) \delta 13.09(\mathrm{~s}, 1 \mathrm{H}), 9.67(\mathrm{~s}, 1 \mathrm{H}), 8.45(\mathrm{~d}, J=3.6$ $\mathrm{Hz}, 2 \mathrm{H}), 8.17(\mathrm{~s}, 1 \mathrm{H}), 8.10-8.00(\mathrm{~m}, 2 \mathrm{H}), 7.95-7.82(\mathrm{~m}, 4 \mathrm{H})$, $7.63-746(\mathrm{~m}, 4 \mathrm{H}), 3.27(\mathrm{~s}, 3 \mathrm{H}), 2.81(\mathrm{~s}, 3 \mathrm{H}), 1.52(\mathrm{~s}, 9 \mathrm{H})$ (note that minor peaks in the ${ }^{1} \mathrm{H}$ NMR are due to the presence of a tautomers in the sample); ${ }^{13} \mathrm{C}\left\{{ }^{1} \mathrm{H}\right\}$ NMR (126 MHz, DMSO- $\left.d_{6}\right) \delta 152.9,150.0$, $149.7,145.2,142.2,140.1,138.1,137.9,134.4,133.9,133.4,131.5$, $129.6,129.5,129.3,129.0,128.7,127.7(2 \times), 127.0,125.7,124.4$, $122.0,120.8,120.3,113.4,79.5,43.5,28.2,21.5$ (note that extra peaks in the ${ }^{13} \mathrm{C}\left\{{ }^{1} \mathrm{H}\right\}$ NMR are due to the presence of tautomers in the sample); $\nu_{\max }\left(\mathrm{cm}^{-1}\right.$, thin film, ATR) $2925(\mathrm{w}), 2848(\mathrm{w}), 1724(\mathrm{~m})$, $1712(\mathrm{~m}), 1603(\mathrm{~s}), 1494(\mathrm{w}), 1367(\mathrm{w}), 1305(\mathrm{~m}), 1238(\mathrm{~m}), 1150$ (s), $1108(\mathrm{w}), 1052(\mathrm{w}), 1025(\mathrm{w}), 958(\mathrm{~m}), 884(\mathrm{~m}), 835(\mathrm{~m}), 764$ (m); HRMS (ESI+/TOF) $m / z[\mathrm{M}+\mathrm{H}]^{+}$calcd for $\mathrm{C}_{31} \mathrm{H}_{31} \mathrm{~N}_{4} \mathrm{O}_{4} \mathrm{~S}$ 555.2066, found 555.2047; $\mathrm{mp} 180.0{ }^{\circ} \mathrm{C}$ (dec).

4-(5-([1,1'-Biphenyl]-4-yl)-2-(2-methyl-4-(methylsulfonyl)phenyl)-1H-imidazol-4-yl)pyridine (61). The title compound was prepared according to general procedure C, using $44(39.0 \mathrm{mg}, 0.10$ mmol, 1.00 equiv), 4-biphenylboronic acid $(25.0 \mathrm{mg}, 0.125 \mathrm{mmol}$, 1.25 equiv), $\mathrm{Pd}(\mathrm{OAc})_{2}(2.3 \mathrm{mg}, 10 \mathrm{~mol} \%$ ), and Aphos (6.7 mg, 24 $\mathrm{mol} \%$ ). Purification by silica gel chromatography, eluting with EtOH in $\mathrm{CHCl}_{3}(21 \mathrm{~cm} \times 10 \mathrm{~mm}$, gradient elution, $0 \% \rightarrow 4 \%, 0.5 \%$ increases, $20 \mathrm{~mL}$ runs, $3-4 \mathrm{~mL}$ fractions) followed by repurification in silica gel using $\mathrm{MeOH}$ in DCM eluent $(21 \mathrm{~cm} \times 10 \mathrm{~mm}$, gradient elution, $0 \% \rightarrow 4.5 \%, 0.5 \%$ increases, $20 \mathrm{~mL}$ runs, $7 \mathrm{~mL}$ fractions), yielded 61 as a pale yellow solid ( $60 \%$ yield, $28.0 \mathrm{mg}, 0.06 \mathrm{mmol}): R_{f}$ $=0.42\left(7 \% \mathrm{EtOH} / \mathrm{CHCl}_{3}\right.$, Dragendorff stain $) ;{ }^{1} \mathrm{H}$ NMR $(500 \mathrm{MHz}$, DMSO $\left.-d_{6} / \mathrm{D}_{2} \mathrm{O} / \mathrm{TFA}\right) \delta 8.60(\mathrm{~d}, J=6.9 \mathrm{~Hz}, 2 \mathrm{H}), 8.09(\mathrm{~d}, J=6.9 \mathrm{~Hz}$,
$2 \mathrm{H}), 7.94(\mathrm{~d}, J=8.2 \mathrm{~Hz}, 1 \mathrm{H}), 7.91(\mathrm{~s}, 1 \mathrm{H}) 7.87-7.81(\mathrm{~m}, 3 \mathrm{H})$, $7.74-7.68(\mathrm{~m}, 4 \mathrm{H}), 7.49(\mathrm{t}, J=7.7 \mathrm{~Hz}, 2 \mathrm{H}), 7.39(\mathrm{t}, J=7.3 \mathrm{~Hz}, 1 \mathrm{H})$; ${ }^{13} \mathrm{C}\left\{{ }^{1} \mathrm{H}\right\}$ NMR $\left(126 \mathrm{MHz}, \mathrm{DMSO}-d_{6} / \mathrm{D}_{2} \mathrm{O} / \mathrm{TFA}\right) \delta 150.9,147.3$, $142.3,141.8,141.5,139.8,139.6,137.3,133.6,131.5,130.9,130.4$, $130.2,130.0,129.0,128.4,128.3,127.5,125.3,123.3,44.2,21.6 ; \nu_{\max }$ $\left(\mathrm{cm}^{-1}\right.$, thin film, ATR) $2925(\mathrm{br}), 2360(\mathrm{w}), 1602(\mathrm{~s}), 1517(\mathrm{w})$, 1480 (w), 1443 (w), 1312 (s), 1213 (w), 1147 (s), 1109 (m), 1075 $(\mathrm{w}), 1075(\mathrm{w}), 999(\mathrm{w}), 958(\mathrm{~m}), 877(\mathrm{w}), 768(\mathrm{~s}), 762(\mathrm{~s}), 744(\mathrm{~s})$, $733(\mathrm{~s}), 700(\mathrm{~m})$; HRMS (ESI+/TOF) $\mathrm{m} / z[\mathrm{M}+\mathrm{H}]^{+}$calcd for $\mathrm{C}_{28} \mathrm{H}_{24} \mathrm{~N}_{3} \mathrm{O}_{2} \mathrm{~S} 466.1589$, found 466.1571; mp $241.0{ }^{\circ} \mathrm{C}$ (dec).

4'-(2-(2-Methyl-4-(methylsulfonyl)phenyl)-5-(pyridin-4-yl)-1Himidazol-4-yl)-[1,1'-biphenyl]-4-carbonitrile (62). The title compound was prepared according to general procedure C, using 44 (39.0 $\mathrm{mg}, 0.10 \mathrm{mmol}, 1.00$ equiv), 4'-(4,4,5,5-tetramethyl-1,3,2-dioxaborolan-2-yl)-[1,1'-biphenyl]-4-carbonitrile $(39.0 \mathrm{mg}, 0.125 \mathrm{mmol}, 1.25$ equiv), $\mathrm{Pd}(\mathrm{OAc})_{2}(2.3 \mathrm{mg}, 10 \mathrm{~mol} \%)$, and Aphos $(6.7 \mathrm{mg}, 24 \mathrm{~mol}$ $\%)$. Purification by silica gel chromatography, eluting with $\mathrm{EtOH}$ in $\mathrm{CHCl}_{3}(21 \mathrm{~cm} \times 10 \mathrm{~mm}$, gradient elution, $0 \% \rightarrow 4 \%, 0.5 \%$ increases, $20 \mathrm{~mL}$ runs, $3-4 \mathrm{~mL}$ fractions) followed by repurification in silica gel using $\mathrm{MeOH}$ in DCM eluent $(21 \mathrm{~cm} \times 10 \mathrm{~mm}$, gradient elution, $0 \%$ $\rightarrow 5 \%, 0.5 \%$ increases, $20 \mathrm{~mL}$ runs, $7 \mathrm{~mL}$ fractions), yielded 62 as a white solid (47\% yield, $23.0 \mathrm{mg}, 0.05 \mathrm{mmol}) ; R_{f}=0.35(7 \% \mathrm{EtOH} /$ $\mathrm{CHCl}_{3}$, Dragendorff stain); ${ }^{1} \mathrm{H}$ NMR (500 MHz, DMSO- $d_{6} / \mathrm{D}_{2} \mathrm{O} /$ TFA) $\delta 8.61(\mathrm{~d}, J=6.8 \mathrm{~Hz}, 1 \mathrm{H}), 8.10(\mathrm{~d}, J=6.8 \mathrm{~Hz}, 1 \mathrm{H}), 7.97-7.88$ $(\mathrm{m}, 8 \mathrm{H}), 7.86(\mathrm{~d}, J=8.1 \mathrm{~Hz}, 1 \mathrm{H}), 7.75(\mathrm{~d}, J=8.3 \mathrm{~Hz}, 1 \mathrm{H}), 3.21(\mathrm{~s}$, $1 \mathrm{H}), 2.71(\mathrm{~s}, 1 \mathrm{H}) ;{ }^{13} \mathrm{C}\left\{{ }^{1} \mathrm{H}\right\}$ NMR $\left(126 \mathrm{MHz}\right.$, DMSO-d $\left.d_{6} / \mathrm{D}_{2} \mathrm{O} / \mathrm{TFA}\right)$ $\delta$ 151.0, 147.5, 144.4, 141.8, 141.4, 140.1, 139.4, 136.9, 133.7, 133.7, $131.8,130.8,130.5,130.2,129.9,128.7,128.4,125.2,123.2,119.7$, 111.2, 44.1, 21.6; $\nu_{\max }\left(\mathrm{cm}^{-1}\right.$, thin film, ATR) $3083(\mathrm{br}), 2846(\mathrm{br})$, $2359(\mathrm{w}), 2225(\mathrm{w}), 1604(\mathrm{~m}), 1499(\mathrm{w}), 1410(\mathrm{w}), 1310(\mathrm{~m}), 1301$ (m), $1150(\mathrm{~s}), 1111(\mathrm{~m}), 1077(\mathrm{w}), 1004(\mathrm{w}), 972(\mathrm{w}), 959(\mathrm{w}), 880$ (w), $825(\mathrm{~s}), 765(\mathrm{~m}), 765(\mathrm{~m}), 745(\mathrm{~m}), 715$ (w), $693(\mathrm{~m})$; HRMS (ESI+/TOF) $m / z[\mathrm{M}+\mathrm{H}]^{+}$calcd for $\mathrm{C}_{29} \mathrm{H}_{23} \mathrm{~N}_{4} \mathrm{O}_{2} \mathrm{~S} 491.1542$, found 491.1530; mp $255.0^{\circ} \mathrm{C}$ (dec).

4-(2-(2-Methyl-4-(methylsulfonyl)phenyl)-4-(5,6,7,8-tetrahydronaphthalen-2-yl)-1H-imidazol-5-yl)pyridine (63). The title compound was prepared according to general procedure C, using $44(39.0$ mg, $0.10 \mathrm{mmol}, 1.00$ equiv), 4,4,5,5-tetramethyl-2-(5,6,7,8-tetrahydronaphthalen-2-yl)-1,3,2-dioxaborolane $(\mathrm{S} 15) \quad(33.0 \mathrm{mg}, 0.125$ mmol, 1.25 equiv), $\mathrm{Pd}(\mathrm{OAc})_{2}(2.3 \mathrm{mg}, 10 \mathrm{~mol} \%)$, and Aphos (6.7 $\mathrm{mg}, 24 \mathrm{~mol} \%)$. Purification by silica gel chromatography, eluting with EtOH in $\mathrm{CHCl}_{3}(21 \mathrm{~cm} \times 10 \mathrm{~mm}$, gradient elution, $0 \% \rightarrow 5.5 \%, 0.5 \%$ increases, $20 \mathrm{~mL}$ runs, $3-4 \mathrm{~mL}$ fractions) followed by repurification in silica gel using $\mathrm{MeOH}$ in DCM eluent $(21 \mathrm{~cm} \times 10 \mathrm{~mm}$, gradient elution, $0 \% \rightarrow 5.5 \%, 0.5 \%$ increases, $20 \mathrm{~mL}$ runs, $7 \mathrm{~mL}$ fractions), yielded 63 as a white solid ( $61 \%$ yield, $27.0 \mathrm{mg}, 0.06 \mathrm{mmol}): R_{f}=0.33$ (7\% EtOH $/ \mathrm{CHCl}_{3}, \mathrm{UV}$, Dragendorff stain); ${ }^{1} \mathrm{H}$ NMR $(500 \mathrm{MHz}$, DMSO- $\left.d_{6} / \mathrm{D}_{2} \mathrm{O} / \mathrm{TFA}\right) \delta 8.61(\mathrm{~d}, J=6.6 \mathrm{~Hz}, 2 \mathrm{H}), 8.08(\mathrm{~d}, J=6.8 \mathrm{~Hz}$, $2 \mathrm{H}), 7.94(\mathrm{~d}, J=8.3 \mathrm{~Hz}, 1 \mathrm{H}), 7.91(\mathrm{~s}, 1 \mathrm{H}), 7.85(\mathrm{~d}, J=8.3 \mathrm{~Hz}, 1 \mathrm{H})$, $7.32(\mathrm{~s}, 1 \mathrm{H}), 7.29(\mathrm{~d}, J=7.9 \mathrm{~Hz}, 1 \mathrm{H}), 7.22(\mathrm{~d}, J=7.9 \mathrm{~Hz}, 1 \mathrm{H}), 3.22$ (s, $3 \mathrm{H}), 2.80-2.73(\mathrm{~m}, 4 \mathrm{H}), 2.71(\mathrm{~s}, 3 \mathrm{H}), 1.78-1.72(\mathrm{~m}, 4 \mathrm{H})$; ${ }^{13} \mathrm{C}\left\{{ }^{1} \mathrm{H}\right\}$ NMR (126 MHz, DMSO- $\left.d_{6} / \mathrm{D}_{2} \mathrm{O} / \mathrm{TFA}\right) \delta 151.2,146.8$, $141.5,141.2,139.6,139.1,138.6,137.8,133.6,131.2,130.6,130.5$, 130.0, 129.9, 126.6, 126.4, 125.0, 122.6, 44.0, 29.3, 29.2, 23.0, 21.5 (note that the signal at $\delta 23.0 \mathrm{ppm}$ in the ${ }^{13} \mathrm{C}\left\{{ }^{1} \mathrm{H}\right\}$ NMR corresponds to two carbons from the tetrahydronaphthalene moiety); $\nu_{\max }\left(\mathrm{cm}^{-1}\right.$, thin film, ATR) 2935 (w), 2856 (w), 1599 (s), 1429 (w), 1309 (s), $1212(\mathrm{w}), 1147(\mathrm{~s}), 1106(\mathrm{~m}), 1076(\mathrm{w}), 998(\mathrm{w}), 963(\mathrm{w}), 952(\mathrm{w})$, 871 (w), 827 (m), 808 (w), 765 (s), 738 (m); HRMS (ESI+/TOF) $m / z[\mathrm{M}+\mathrm{H}]^{+}$calcd for $\mathrm{C}_{26} \mathrm{H}_{26} \mathrm{~N}_{3} \mathrm{O}_{2} \mathrm{~S} 444.1746$, found 444.1761; mp $252.0^{\circ} \mathrm{C}(\mathrm{dec})$.

4-(4-(2,3-Dihydrobenzo[b][1,4]dioxin-6-yl)-2-(2-methyl-4(methylsulfonyl)phenyl)-1H-imidazol-5-yl)pyridine (64). The title compound was prepared according to general procedure $C$, using 44 (39.0 mg, $0.10 \mathrm{mmol}, 1.00$ equiv), 1,4-benzodioxane-6-boronic acid ( $25.0 \mathrm{mg}, 0.125 \mathrm{mmol}, 1.25$ equiv), $\mathrm{Pd}(\mathrm{OAc})_{2}(2.3 \mathrm{mg}, 10 \mathrm{~mol} \%)$, and Aphos (6.7 mg, $24 \mathrm{~mol} \%)$. Purification by silica gel chromatography, eluting with EtOH in $\mathrm{CHCl}_{3}(21 \mathrm{~cm} \times 10 \mathrm{~mm}$, gradient elution, $0 \% \rightarrow 5 \%, 0.5 \%$ increases, $20 \mathrm{~mL}$ runs, $3-4 \mathrm{~mL}$ fractions) followed by repurification in silica gel using $\mathrm{MeOH}$ in 
DCM eluent $(15 \mathrm{~cm} \times 10 \mathrm{~mm}$, gradient elution, $0 \% \rightarrow 6 \%, 0.5 \%$ increases, $20 \mathrm{~mL}$ runs, $7 \mathrm{~mL}$ fractions), yielded 64 as a pale yellow solid $(60 \%$ yield, $27.0 \mathrm{mg}, 0.06 \mathrm{mmol}): R_{f}=0.37\left(7 \% \mathrm{EtOH} / \mathrm{CHCl}_{3}\right.$, Dragendorff stain); ${ }^{1} \mathrm{H}$ NMR (500 MHz, DMSO- $\left.d_{6} / \mathrm{D}_{2} \mathrm{O} / \mathrm{TFA}\right) \delta$ $8.59(\mathrm{~d}, J=7.0 \mathrm{~Hz}, 2 \mathrm{H}), 8.06(\mathrm{~d}, J=7.0 \mathrm{~Hz}, 2 \mathrm{H}), 7.91(\mathrm{~d}, J=8.2 \mathrm{~Hz}$, $1 \mathrm{H}), 7.89(\mathrm{~d}, J=1.4,1 \mathrm{H}) 7.84(\mathrm{dd}, J=8.2,1.8 \mathrm{~Hz}, 1 \mathrm{H}), 7.10(\mathrm{~d}, J=$ 2.1, $1 \mathrm{H}), 7.05(\mathrm{dd} J=8.3,2.1 \mathrm{~Hz}, 1 \mathrm{H}), 7.00(\mathrm{~d}, J=8.3 \mathrm{~Hz}, 1 \mathrm{H})$, $4.30-4.25(\mathrm{~m}, 4 \mathrm{H}), 3.20(\mathrm{~s}, 3 \mathrm{H}), 2.67(\mathrm{~s}, 3 \mathrm{H}) ;{ }^{13} \mathrm{C}\left\{{ }^{1} \mathrm{H}\right\}$ NMR $(126$ $\left.\mathrm{MHz}, \mathrm{DMSO}-d_{6} / \mathrm{D}_{2} \mathrm{O} / \mathrm{TFA}\right) \delta 150.9,146.8,145.6,144.6,141.7$, $141.5,139.5,137.2,133.5,130.9,130.8,130.2,125.2,123.0,123.0$, $122.1,118.9,118.4,65.1,64.9,44.1,21.5 ; \nu_{\max }\left(\mathrm{cm}^{-1}\right.$, thin film, ATR $)$ 2668 (br), 2360 (w), 1603 (s), 1541 (w), 1512 (w), 1489 (w), 1461 $(\mathrm{w}), 1442(\mathrm{w}), 1311(\mathrm{~s}), 1287(\mathrm{~s}), 1253(\mathrm{~m}), 1154(\mathrm{~s}), 1112(\mathrm{w})$, $1097(\mathrm{w}), 1063(\mathrm{~s}), 1049(\mathrm{w}), 1006(\mathrm{w}), 977(\mathrm{w}), 965(\mathrm{w}), 951(\mathrm{~m})$, $931(\mathrm{w}), 893(\mathrm{w}), 875(\mathrm{w}), 865(\mathrm{~m}), 841(\mathrm{w}), 830(\mathrm{~s}), 764(\mathrm{~m}), 741$ (m); HRMS (ESI+/TOF) $m / z[\mathrm{M}+\mathrm{H}]^{+}$calcd for $\mathrm{C}_{24} \mathrm{H}_{22} \mathrm{~N}_{3} \mathrm{O}_{4} \mathrm{~S}$ 448.1331, found 448.1315; mp $299.0{ }^{\circ} \mathrm{C}$ (dec).

One-Pot Miyaura Borylation-Suzuki Coupling. 7-(2-(2Methyl-4-(methylsulfonyl)phenyl)-5-(pyridin-4-yl)-1H-imidazol-4yl)quinoline (67). A culture tube $(13 \mathrm{~mm} \times 100 \mathrm{~mm}, 9 \mathrm{~mL})$ was charged with 7-bromoquinoline (65) $(42.0 \mathrm{mg}, 0.20 \mathrm{mmol}, 2.00$ equiv), $\mathrm{B}_{2}(\text { pin })_{2}(80.0 \mathrm{mg}, 0.30 \mathrm{mmol}, 3.00$ equiv), KOAc $(59.0 \mathrm{mg}$, $0.60 \mathrm{mmol}, 6.00$ equiv), and a magnetic stir bar under nitrogen. Degassed DME $(0.15 \mathrm{~mL})$ was added followed by a premixed solution of $\mathrm{Pd}(\mathrm{OAc})_{2}(2.4 \mathrm{mg}, 0.011 \mathrm{mmol}, 5 \mathrm{~mol} \%$ relative to 65$)$ and Aphos (7.1 mg, $0.025 \mathrm{mmol}, 12 \%$ relative to 65) in DME (0.35 $\mathrm{mL}$ ). The reaction mixture was stirred in a preheated aluminum block at $80{ }^{\circ} \mathrm{C}$ for $2 \mathrm{~h}$. After consumption of the starting material, indicated by TLC analysis ( $\left.30 \% \mathrm{EtOAc/hexane,} \mathrm{KMnO}_{4}\right)$, the reaction mixture was cooled to room temperature, the culture tube was opened under a nitrogen flow, and $44(39.0 \mathrm{mg}, 0.10 \mathrm{mmol}, 1.00$ equiv) was added followed by addition of a premixed solution of $\mathrm{Pd}(\mathrm{OAc})_{2}(1.2 \mathrm{mg}$, $0.005 \mathrm{mmol}, 5 \mathrm{~mol} \%$ relative to 44$)$ and Aphos $(3.6 \mathrm{mg}, 0.01 \mathrm{mmol}$, $12 \mathrm{~mol} \%$ relative to 44$)$ in DME $(0.15 \mathrm{~mL})$. Then, DME $(0.10 \mathrm{~mL})$ and $1.2 \mathrm{M} \mathrm{K}_{2} \mathrm{CO}_{3}$ aqueous solution $(0.25 \mathrm{~mL}, 0.30 \mathrm{mml}, 3.00$ equiv) were added, and the reaction mixture was purged with nitrogen for 5 $\mathrm{min}$. The reaction mixture was stirred in a preheated aluminum block at $80{ }^{\circ} \mathrm{C}$ for $18 \mathrm{~h}$. After consumption of 44 , indicated by TLC analysis (7\% EtOH/ $\mathrm{CHCl}_{3}$, Dragendorff), the reaction mixture was allowed to reach room temperature and it was diluted with $10 \% \mathrm{MeOH} / \mathrm{DCM}$ $(\sim 7 \mathrm{~mL})$, filtered through a pad $(20 \mathrm{~mm}$ diameter $)$ composed of Celite (top, $1 \mathrm{~cm}$ ) and silica gel (bottom, $3 \mathrm{~cm}$ ). The pad was washed with $10 \% \mathrm{MeOH} / \mathrm{DCM}(25-50 \mathrm{~mL})$, and the filtrate was concentrated under reduced pressure. The crude product was adsorbed over basic alumina and purified by silica column chromatography, eluting with EtOH in $\mathrm{CHCl}_{3}(21 \mathrm{~cm} \times 10 \mathrm{~mm}$, gradient elution, $0 \% \rightarrow 7 \%, 0.5 \%$ increases, $20 \mathrm{~mL}$ runs, $3-4 \mathrm{~mL}$ fractions) followed by repurification in silica gel using $\mathrm{MeOH}$ in DCM eluent $(21 \mathrm{~cm} \times 10 \mathrm{~mm}$, gradient elution, $0 \% \rightarrow 8 \%, 0.5 \%$ increases, $20 \mathrm{~mL}$ runs, $7 \mathrm{~mL}$ fractions), to yield 67 as a white solid ( $50 \%$ yield, $22.0 \mathrm{mg}, 0.05 \mathrm{mmol}): R_{f}=0.45\left(7 \% \mathrm{EtOH} / \mathrm{CHCl}_{3}\right.$, Dragendorff stain); ${ }^{1} \mathrm{H}$ NMR (500 MHz, DMSO- $\left.d_{6} / \mathrm{D}_{2} \mathrm{O} / \mathrm{TFA}\right) \delta$ $9.22(\mathrm{~d}, J=5.2 \mathrm{~Hz}, 1 \mathrm{H}), 9.12(\mathrm{~d}, J=8.4 \mathrm{~Hz}, 1 \mathrm{H}), 8.66(\mathrm{~d}, J=6.8 \mathrm{~Hz}$, $2 \mathrm{H}), 8.44(\mathrm{~s}, 1 \mathrm{H}), 8.41(\mathrm{~d}, J=8.6 \mathrm{~Hz}, 1 \mathrm{H}), 8.13(\mathrm{~d}, J=6.8 \mathrm{~Hz}, 2 \mathrm{H})$, $8.09(\mathrm{~d}, J=8.5 \mathrm{~Hz}, 1 \mathrm{H}), 8.07-8.03(\mathrm{~m}, 1 \mathrm{H}), 7.99(\mathrm{~d}, J=8.1 \mathrm{~Hz}$, $1 \mathrm{H}), 7.94(\mathrm{~s}, 1 \mathrm{H}), 7.89(\mathrm{~d}, J=8.1 \mathrm{~Hz}, 1 \mathrm{H}), 3.23(\mathrm{~s}, 3 \mathrm{H}), 2.74(\mathrm{~s}$, $3 \mathrm{H}) ;{ }^{13} \mathrm{C}\left\{{ }^{1} \mathrm{H}\right\}$ NMR $\left(126 \mathrm{MHz}, \mathrm{DMSO}-d_{6} / \mathrm{D}_{2} \mathrm{O} / \mathrm{TFA}\right) \delta 150.0$, $148.5,147.3,146.0,142.1,141.5,139.7,139.5,135.9,135.5,133.8$, $132.5,131.0,130.8,130.2,130.2,129.3,125.2,124.1,123.3,122.0$, 44.1, 21.5; $\nu_{\max }\left(\mathrm{cm}^{-1}\right.$, thin film, ATR) $2922(\mathrm{w}), 2845(\mathrm{w}), 1614$ (w), $1584(w), 1509(w), 1490(w), 1449(w), 1303(s), 1210(w)$, $1155(\mathrm{~m}), 1141(\mathrm{~s}), 1104(\mathrm{~m}), 1073(\mathrm{w}), 975(\mathrm{w}), 958(\mathrm{w}), 880(\mathrm{~m})$, 837 (s), 765 (s), $742(\mathrm{~m})$; HRMS (ESI+/TOF) $\mathrm{m} / z[\mathrm{M}+\mathrm{H}]^{+}$calcd for $\mathrm{C}_{25} \mathrm{H}_{21} \mathrm{~N}_{4} \mathrm{O}_{2} \mathrm{~S} 441.1385$, found 441.1372; mp $300.0{ }^{\circ} \mathrm{C}$ (dec).

tert-Butyl 4-(4-Bromo-5-(pyridin-4-yl)-1H-imidazol-2-yl)piperidine-1-carboxylate (69). Following a modified literature procedure, ${ }^{24}$ a $25 \mathrm{~mL}$ round-bottom flask was charged with 36 (437 mg, $1.33 \mathrm{mmol}, 1.00$ equiv), dry DCM $(5.9 \mathrm{~mL})$, dry pyridine $(1.5 \mathrm{~mL})$, and a magnetic stir bar under an inert atmosphere. The reaction flask was covered with aluminum foil, and the reaction mixture was cooled to $0{ }^{\circ} \mathrm{C}$ in an ice/water bath and stirred for 15 min. Solid $\mathrm{Py} \cdot \mathrm{HBr} \cdot \mathrm{Br}_{2}$ (pyridinium hydrobromide perbromide, 520 $\mathrm{mg}, 1.46 \mathrm{mmol}, 1.10$ equiv) was added in portions, by briefly removing the Suba seal, and the reaction mixture was stirred at $0{ }^{\circ} \mathrm{C}$ for $1 \mathrm{~h}$. After consumption of the starting material, indicated by TLC analysis (100\% EtOAc, Dragendorff), the solvent was removed in the rotaevaporator. The residue was partitioned between $1 \mathrm{M}$ aq $\mathrm{NaHSO}_{3}$ $(1 \times 30 \mathrm{~mL})$ and $\mathrm{CHCl}_{3}(1 \times 30 \mathrm{~mL})$. The phases were separated, and the aqueous layer was extracted with $\mathrm{CHCl}_{3}(3 \times 15 \mathrm{~mL})$. The organic phases were combined, dried over $\mathrm{MgSO}_{4}$, filtered, and concentrated in the rotaevaporator. Purification by silica gel chromatography, eluting with $\mathrm{MeOH}$ in $\mathrm{CHCl}_{3}(13 \mathrm{~cm} \times 30 \mathrm{~mm}$, gradient elution, $4 \% \rightarrow 6 \%$, $0.5 \%$ increases, $80 \mathrm{~mL}$ runs, $20 \mathrm{~mL}$ fractions) followed by repurification in silica gel using EtOAc/EtOH (3:1) in hexanes $(13 \mathrm{~cm} \times 30 \mathrm{~mm}$, isocratic elution, $50 \%$ EtOAc/ EtOH (3:1)/hexane, $400 \mathrm{~mL}$ run, $20 \mathrm{~mL}$ fractions), yielded a light yellow gum, to which precipitation was induced with pentane to afford 69 as a pale yellow solid (95\% yield, $513 \mathrm{mg}, 1.26 \mathrm{mmol}$ ): $R_{f}=$ 0.37 (50\% EtOAc:EtOH (3:1)/hexaneanes, UV, Dragendorff): $R_{f}=$ 0.17 (5\% MeOH/DCM, UV, Dragendorff); ${ }^{1} \mathrm{H}$ NMR (500 MHz, $\left.\mathrm{CDCl}_{3}\right) \delta 12.10(\mathrm{br} \mathrm{s}, 1 \mathrm{H}), 8.52(\mathrm{~s}, 2 \mathrm{H}), 7.76(\mathrm{~s}, 2 \mathrm{H}), 4.14(\mathrm{~d}, J=$ $11.4 \mathrm{~Hz}, 2 \mathrm{H}), 2.97-2.88(\mathrm{~m}, 1 \mathrm{H}), 2.88-2.68(\mathrm{~m}, 2 \mathrm{H}), 1.92(\mathrm{~d}, J=$ $11.5 \mathrm{~Hz}, 2 \mathrm{H}), 1.84-1.64(\mathrm{~m}, 2 \mathrm{H}), 1.43(\mathrm{~s}, 9 \mathrm{H}) ;{ }^{13} \mathrm{C}\left\{{ }^{1} \mathrm{H}\right\}$ NMR $(126$ $\left.\mathrm{MHz} \mathrm{CDCl}_{3}\right) \delta 154.8,153.2,149.6,120.4,80.3,43.9,36.7,30.8,28.6$ (note that, due to slow relaxation, some ${ }^{13} \mathrm{C}\left\{{ }^{1} \mathrm{H}\right\}$ NMR signals were not identified in the spectra; ${ }^{28}$ specifically, the ${ }^{13} \mathrm{C}\left\{{ }^{1} \mathrm{H}\right\}$ NMR data for compound 69 lacks three of the 12 expected signals) $\nu_{\max }\left(\mathrm{cm}^{-1}\right.$, thin film, ATR) 2875 (br), 1679 (s), 1603 (s), 1580 (w), 1519 (w), 1367 (m), $1276(\mathrm{~m}), 1233(\mathrm{~s}), 1164(\mathrm{~s}), 1125(\mathrm{~m}), 1063(\mathrm{w}), 1045(\mathrm{w})$, $1003(\mathrm{~m}), 981(\mathrm{~m}), 935(\mathrm{~m}), 874(\mathrm{w}), 821(\mathrm{~m}), 723(\mathrm{w}), 693(\mathrm{~m})$; HRMS (ESI+/TOF) $m / z[\mathrm{M}+\mathrm{H}]^{+}$calcd for $\mathrm{C}_{18} \mathrm{H}_{24} \mathrm{BrN}_{4} \mathrm{O}_{2}$ 407.1083, 409.1064, found 407.1051, 409.1126; mp $197.0{ }^{\circ} \mathrm{C}$ (dec).

tert-Butyl 4-(4-(6-methoxynaphthalen-2-yl)-5-(pyridin-4-yl)- $1 \mathrm{H}$ imidazol-2-yl)piperidine-1-carboxylate (71). The title compound was prepared according to general procedure C, using $69(41.0 \mathrm{mg}$, $0.10 \mathrm{mmol}, 1.00$ equiv), 2-(6-methoxynaphthalen-2-yl)-4,4,5,5tetramethyl-1,3,2-dioxaborolane (S16) $(52.0 \mathrm{mg}, 0.175 \mathrm{mmol}, 1.75$ equiv), $\mathrm{Pd}(\mathrm{OAc})_{2}(2.3 \mathrm{mg}, 10 \mathrm{~mol} \%)$, and Aphos $(6.7 \mathrm{mg}, 24 \mathrm{~mol}$ $\%)$. Purification by silica gel chromatography, eluting with $\mathrm{EtOH}$ in $\mathrm{CHCl}_{3}(20 \mathrm{~cm} \times 15 \mathrm{~mm}$, gradient elution, $0 \% \rightarrow 4.5 \%, 0.5 \%$ increases, $25 \mathrm{~mL}$ runs, $5 \mathrm{~mL}$ fractions then isocratic elution, $4.5 \%$ $\mathrm{EtOH} / \mathrm{CHCl}_{3}, 50 \mathrm{~mL}$ run, $5 \mathrm{~mL}$ fractions), yielded 71 as a pale yellow solid ( $89 \%$ yield, $43.0 \mathrm{mg}$, $0.89 \mathrm{mmol}): R_{f}=0.40(7 \% \mathrm{EtOH} /$ $\mathrm{CHCl}_{3}, \mathrm{UV}$, Dragendorff stain); ${ }^{1} \mathrm{H}$ NMR $\left(250 \mathrm{MHz}, \mathrm{CDCl}_{3}\right) \delta$ $10.07($ br s, $1 \mathrm{H}), 8.41(\mathrm{~d}, J=5.2 \mathrm{~Hz}, 2 \mathrm{H}), 7.90-7.77(\mathrm{~m}, 1 \mathrm{H}), 7.72$ $(\mathrm{d}, J=8.6 \mathrm{~Hz}, 1 \mathrm{H}), 7.67(\mathrm{~d}, J=8.8 \mathrm{~Hz}, 1 \mathrm{H}), 7.59-7.36(\mathrm{~m}, 3 \mathrm{H})$, $7.21-7.11(\mathrm{~m}, 2 \mathrm{H}), 4.30-4.12(\mathrm{~m}, 2 \mathrm{H}), 3.93(\mathrm{~s}, 3 \mathrm{H}), 3.00(\mathrm{tt}, J=$ 11.7, $3.6 \mathrm{~Hz}, 1 \mathrm{H}), 2.92-2.73(\mathrm{~m}, 2 \mathrm{H}), 2.11-1.95(\mathrm{~m}, 2 \mathrm{H}), 1.77(\mathrm{dq}$, $J=3.7,12.4 \mathrm{~Hz}, 1 \mathrm{H}), 1.45(\mathrm{~s}, 9 \mathrm{H}) ;{ }^{13} \mathrm{C}\left\{{ }^{1} \mathrm{H}\right\}$ NMR $(126 \mathrm{MHz}$, $\left.\mathrm{CDCl}_{3}\right) \delta 158.5,154.8,151.3,150.2,149.8,142.8,134.4,133.6,129.6$, $129.4,129.0,127.7,127.2,127.1,126.8,125.8,121.5,121.3,119.8$, $105.9,79.9,55.5,36.5,31.0,29.8,28.6 ; \nu_{\max }\left(\mathrm{cm}^{-1}\right.$, thin film, ATR $)$ 2930 (br), 1693 (s), 1601 (s), $1536(\mathrm{w}), 1418(\mathrm{~m}), 1391(\mathrm{w}), 1366$ (w), $1273(\mathrm{~m}), 1249(\mathrm{w}), 1210(\mathrm{~m}), 1165(\mathrm{~s}), 1123(\mathrm{~m}), 1085(\mathrm{~m})$, $1030(\mathrm{w}), 1007(\mathrm{w}), 99(\mathrm{w}), 857(\mathrm{w}), 831(\mathrm{~m}), 693(\mathrm{w}), 667(\mathrm{w})$; HRMS (ESI+/TOF) $m / z[\mathrm{M}+\mathrm{H}]^{+}$calcd for $\mathrm{C}_{29} \mathrm{H}_{33} \mathrm{~N}_{4} \mathrm{O}_{3} 485.2553$, found 485.2537; mp $193.0{ }^{\circ} \mathrm{C}(\mathrm{dec})$. Spectroscopic data are in accordance with the literature. ${ }^{11}$

Synthesis of GSK3037619A (72). N-Boc piperidine-substituted imidazole 71 (12.0 mg, $0.03 \mathrm{mmol}, 1.0$ equiv) was dissolved in DCM $(0.25 \mathrm{~mL}, 0.1 \mathrm{M})$ under a nitrogen atmosphere. Trifluoroacetic acid (39 $\mu \mathrm{L}, 0.50 \mathrm{mmol}, 20$ equiv) was added, and the reaction mixture was allowed to stir for $1 \mathrm{~h}$ and was followed by TLC $(10 \% \mathrm{MeOH} /$ $\left.\mathrm{NH}_{4} \mathrm{OH}(10: 1) / \mathrm{DCM}\right)$. After consumption of the starting material, the solvent and excess trifluoroacetic acid were removed in vacuo and the residue was dissolved in anhydrous $\mathrm{MeCN}(1 \mathrm{~mL}, 0.03 \mathrm{M})$ under a nitrogen atmosphere. Then $\mathrm{Et}_{3} \mathrm{~N}(5.3 \mu \mathrm{L}, 0.04 \mathrm{mmol}, 1.5$ equiv) was added followed by a $37 \%$ aqueous formaldehyde solution $(14 \mu \mathrm{L}$, $0.19 \mathrm{mmol}, 7.5$ equiv), and the reaction mixture was left to stir for $1 \mathrm{~h}$ at room temperature. $\mathrm{Na}(\mathrm{OAc})_{3} \mathrm{BH}(14.0 \mathrm{mg}, 0.06 \mathrm{mmol}, 2.5$ equiv $)$ 
was added, and the reaction mixture was stirred for $18 \mathrm{~h}$. The solvent was removed under reduced pressure, and the residue was diluted in $10 \% \mathrm{MeOH} / \mathrm{NH}_{4} \mathrm{OH}(10: 1) / \mathrm{CHCl}_{3}$, filtered through a short $(1 \mathrm{~cm}$ $\times 15 \mathrm{~mm})$ pad of silica gel, which was washed with $10 \% \mathrm{MeOH} /$ $\mathrm{NH}_{4} \mathrm{OH}(10: 1) / \mathrm{CHCl}_{3}$ until the product was eluted completely. The solvent was concentrated, resulting in a yellow residue. Purification by silica gel chromatography, eluting with $\mathrm{MeOH} / \mathrm{NH}_{4} \mathrm{OH}$ (10:1) in $\mathrm{CHCl}_{3}\left(4 \mathrm{~cm} \times 15 \mathrm{~mm}\right.$, isocratic elution, $10 \% \mathrm{MeOH} / \mathrm{NH}_{4} \mathrm{OH}$ $(10: 1) / \mathrm{CHCl}_{3}, 50 \mathrm{~mL}$ run, $2 \mathrm{~mL}$ fractions), yielded a white solid, which was triturated with $\mathrm{Et}_{2} \mathrm{O} /$ hexanes $(2: 8)(3 \times 5 \mathrm{~mL})$ to afford 72 as a white solid ( $80 \%$ yield, $8 \mathrm{mg}, 0.02 \mathrm{mmol}): R_{f}=0.40(10 \%$ $\mathrm{MeOH} / \mathrm{NH}_{4} \mathrm{OH}(10: 1) / \mathrm{CHCl}_{3}$, UV, Dragendorff stain); ${ }^{1} \mathrm{H}$ NMR $\left(600 \mathrm{MHz}, \mathrm{CD}_{3} \mathrm{OD}\right) \delta 8.36(\mathrm{~d}, J=6.2 \mathrm{~Hz}, 2 \mathrm{H}), 7.88(\mathrm{~s}, 1 \mathrm{H}), 7.82(\mathrm{~d}$, $J=8.5 \mathrm{~Hz}, 1 \mathrm{H}), 7.75(\mathrm{~d}, J=8.9 \mathrm{~Hz}, 1 \mathrm{H}), 7.49(\mathrm{~d}, J=6.2 \mathrm{~Hz}, 2 \mathrm{H})$, $7.43(\mathrm{dd}, J=1.7,8.6 \mathrm{~Hz}, 1 \mathrm{H}), 7.28(\mathrm{~d}, J=2.5 \mathrm{~Hz}, 1 \mathrm{H}), 7.17(\mathrm{dd}, J=$ 9.0, $2.7 \mathrm{~Hz}, 1 \mathrm{H}), 3.93(\mathrm{~s}, 3 \mathrm{H}), 3.02(\mathrm{~d}, J=11.8 \mathrm{~Hz}, 2 \mathrm{H}), 2.87(\mathrm{tt}, J=$ 12.0, 3.9, Hz, 1H), $2.34(\mathrm{~s}, 3 \mathrm{H}), 2.19(\mathrm{dt}, J=11.9,2.0 \mathrm{~Hz}, 2 \mathrm{H}), 2.06$ $(\mathrm{d}, J=11.1 \mathrm{~Hz}, 2 \mathrm{H}), 1.97(\mathrm{dq}, J=12.6,3.4 \mathrm{~Hz}, 2 \mathrm{H}) ;{ }^{13} \mathrm{C}\left\{{ }^{1} \mathrm{H}\right\} \mathrm{NMR}$ $\left(151 \mathrm{MHz}, \mathrm{CD}_{3} \mathrm{OD}\right) \delta 159.9,154.1,150.0,136.0,130.6,130.3,128.6$, 128.6, 127.9, 123.0, 120.6, 106.8, 56.4, 55.8, 46.4, 36.9, 31.7; $\nu_{\max }$ $\left(\mathrm{cm}^{-1}\right.$, thin film, ATR) $3010(\mathrm{br}), 2939(\mathrm{w}), 2848(\mathrm{w}), 2792(\mathrm{w})$, $1630(\mathrm{w}), 1601(\mathrm{~s}), 1535(\mathrm{w}), 1493(\mathrm{w}), 1465(\mathrm{w}), 1379(\mathrm{w}), 1270$ (m), $1209(\mathrm{w}), 1181(\mathrm{w}), 1164(\mathrm{w}), 1127(\mathrm{w}), 1066(\mathrm{w}), 1029(\mathrm{w})$, $994(\mathrm{w}), 832(\mathrm{w}), 753(\mathrm{w}), 695(\mathrm{w})$; HRMS (ESI+/TOF) $m / z[\mathrm{M}+$ $\mathrm{H}]^{+}$calcd for $\mathrm{C}_{25} \mathrm{H}_{27} \mathrm{~N}_{4} \mathrm{O} 399.2185$, found 399.2201; mp $262.0{ }^{\circ} \mathrm{C}$ (dec).

\section{ASSOCIATED CONTENT}

\section{S Supporting Information}

The Supporting Information is available free of charge on the ACS Publications website at DOI: 10.1021/acs.joc.9b01844.

${ }^{1} \mathrm{H}$ and ${ }^{13} \mathrm{C}\left\{{ }^{1} \mathrm{H}\right\}$ NMR spectra for compounds 5-31, 32, $36,43,44,46-64,67,69,71$, and 72, optimization tables, and synthetic procedures for compounds S1-S16 (PDF)

\section{AUTHOR INFORMATION}

\section{Corresponding Author}

*E-mail: rapilli@unicamp.br.

\section{ORCID}

Ronaldo A. Pilli: 0000-0002-5919-7763

\section{Notes}

The authors declare no competing financial interest.

\section{ACKNOWLEDGMENTS}

We thank the Brazilian agencies FAPESP (2013/07607-8 and 2019/13104-5) and CNPq (131263/2017-0) for financial support. The SGC is a registered charity (1097737) that receives funds from AbbVie, Bayer Pharma AG, Boehringer Ingelheim, Canada Foundation for Innovation, Eshelman Institute for Innovation, Genome Canada, Innovative Medicines Initiative (EU/EFPIA) [ULTRA-DD grant no. 115766], Janssen, Merck KGaA Darmstadt Germany, MSD, Novartis Pharma AG, Ontario Ministry of Economic Development and Innovation, Pfizer, Takeda, and Wellcome Trust [106169/ ZZ14/Z]. We thank Dr. Ricardo Serafim for helpful discussions.

\section{REFERENCES}

(1) Blakemore, D. C.; Castro, L.; Churcher, I.; Rees, D. C.; Thomas, A. W.; Wilson, D. M.; Wood, A. Organic Synthesis Provides Opportunities to Transform Drug Discovery. Nat. Chem. 2018, 10 (4), 383-394.
(2) Schreiber, S. L. Organic Synthesis toward Small-Molecule Probes and Drugs. Proc. Natl. Acad. Sci. U. S. A. 2011, 108 (17), 6699-6702.

(3) Anderson, E. B.; Long, T. E. Imidazole- and ImidazoliumContaining Polymers for Biology and Material Science Applications. Polymer 2010, 51 (12), 2447-2454.

(4) Green, M. D.; Long, T. E. Designing Imidazole-Based Ionic Liquids and Ionic Liquid Monomers for Emerging Technologies. Polym. Rev. 2009, 49 (4), 291-314.

(5) Amarasekara, A. S. Acidic Ionic Liquids. Chem. Rev. 2016, 116 (10), 6133-6183.

(6) Zhang, L.; Peng, X.-M.; Damu, G. L. V.; Geng, R.-X.; Zhou, C.H. Comprehensive Review in Current Developments of ImidazoleBased Medicinal Chemistry. Med. Res. Rev. 2014, 34 (2), 340-437.

(7) Sakemi, S.; Sun, H. H. Nortopsentins A, B, and C. Cytotoxic and Antifungal Imidazolediylbis[Indoles] from the Sponge Spongoaorites Ruetzleri. J. Org. Chem. 1991, 56 (13), 4304-4307.

(8) Rani, N.; Sharma, A.; Singh, R. Trisubstituted Imidazole Synthesis: A Review. Mini-Rev. Org. Chem. 2014, 12 (1), 34-65.

(9) Bellina, F.; Rossi, R. Regioselective Functionalization of the Imidazole Ring via Transition Metal-Catalyzed C-N and C-C Bond Forming Reactions. Adv. Synth. Catal. 2010, 352 (8), 1223-1276.

(10) Liverton, N. J.; Butcher, J. W.; Claiborne, C. F.; Claremon, D. A.; Libby, B. E.; Nguyen, K. T.; Pitzenberger, S. M.; Selnick, H. G.; Smith, G. R.; Tebben, A.; et al. Design and Synthesis of Potent, Selective, and Orally Bioavailable Tetrasubstituted Imidazole Inhibitors of P38 Mitogen-Activated Protein Kinase. J. Med. Chem. 1999, 42 (12), 2180-2190.

(11) Lee, C. F.; Holownia, A.; Bennett, J. M.; Elkins, J. M.; St. Denis, J. D.; Adachi, S.; Yudin, A. K. Oxalyl Boronates Enable Modular Synthesis of Bioactive Imidazoles. Angew. Chem., Int. Ed. 2017, 56 (22), 6264-6267.

(12) Selig, R.; Goettert, M.; Schattel, V.; Schollmeyer, D.; Albrecht, W.; Laufer, S. A Frozen Analogue Approach to Aminopyridinylimidazoles Leading to Novel and Promising P38 MAP Kinase Inhibitors. J. Med. Chem. 2012, 55 (19), 8429-8439.

(13) Günther, M.; Lategahn, J.; Juchum, M.; Döring, E.; Keul, M.; Engel, J.; Tumbrink, H. L.; Rauh, D.; Laufer, S. Trisubstituted Pyridinylimidazoles as Potent Inhibitors of the Clinically Resistant L858R/T790M/C797S EGFR Mutant: Targeting of Both Hydrophobic Regions and the Phosphate Binding Site. J. Med. Chem. 2017, 60 (13), 5613-5637.

(14) Tan, J.; Chen, Y.; Li, H.; Yasuda, N. Suzuki-Miyaura CrossCoupling Reactions of Unprotected Haloimidazoles. J. Org. Chem. 2014, 79 (18), 8871-8876.

(15) Elkins, J. M.; Fedele, V.; Szklarz, M.; Abdul Azeez, K. R.; Salah, E.; Mikolajczyk, J.; Romanov, S.; Sepetov, N.; Huang, X. P.; Roth, B. L.; et al. Comprehensive Characterization of the Published Kinase Inhibitor Set. Nat. Biotechnol. 2016, 34 (1), 95-103.

(16) Belkina, N. V.; Liu, Y.; Hao, J.-J.; Karasuyama, H.; Shaw, S. LOK Is a Major ERM Kinase in Resting Lymphocytes and Regulates Cytoskeletal Rearrangement through ERM Phosphorylation. Proc. Natl. Acad. Sci. U. S. A. 2009, 106 (12), 4707-4712.

(17) Kuramochi, S.; Moriguchi, T.; Kuida, K.; Endo, J.; Semba, K.; Nishida, E.; Karasuyama, H. LOK Is a Novel Mouse STE20-like Protein Kinase That Is Expressed Predominantly in Lymphocytes. J. Biol. Chem. 1997, 272 (36), 22679-22684.

(18) Viswanatha, R.; Ohouo, P. Y.; Smolka, M. B.; Bretscher, A. Local Phosphocycling Mediated by LOK/SLK Restricts Ezrin Function to the Apical Aspect of Epithelial Cells. J. Cell Biol. 2012, 199 (6), 969-984.

(19) Endo, J.; Toyama-Sorimachi, N.; Taya, C.; KuramochiMiyagawa, S.; Nagata, K.; Kuida, K.; Takashi, T.; Yonekawa, H.; Yoshizawa, Y.; Miyasaka, N.; et al. Deficiency of a STE20/PAK Family Kinase LOK Leads to the Acceleration of LFA-1 Clustering and Cell Adhesion of Activated Lymphocytes. FEBS Lett. 2000, 468 (2-3), 234-238.

(20) Johnson, N. W.; Semones, M.; Adams, J. L.; Hansbury, M.; Winkler, J. Optimization of Triarylimidazoles for Tie2: Influence of 
Conformation on Potency. Bioorg. Med. Chem. Lett. 2007, 17 (20), 5514-5517.

(21) Niculescu-Duvaz, D.; Niculescu-Duvaz, I.; Suijkerbuijk, B. M. J. M.; Ménard, D.; Zambon, A.; Davies, L.; Pons, J. F.; Whittaker, S.; Marais, R.; Springer, C. J. Potent BRAF Kinase Inhibitors Based on 2,4,5-Trisubstituted Imidazole with Naphthyl and Benzothiophene 4Substituents. Bioorg. Med. Chem. 2013, 21 (5), 1284-1304.

(22) Floyd, M. B.; Du, M. T.; Fabio, P. F.; Jacob, L. A.; Johnson, B. D. The Oxidation of Acetophenones to Arylglyoxals with Aqueous Hydrobromic Acid in Dimethyl Sulfoxide. J. Org. Chem. 1985, 50 (25), 5022-5027.

(23) Zuliani, V.; Cocconcelli, G.; Fantini, M.; Ghiron, C.; Rivara, M. A Practical Synthesis of 2,4(5)-Diarylimidazoles from Simple Building Blocks. J. Org. Chem. 2007, 72 (12), 4551-4553.

(24) Vernier, J.-M.; O’Connor, P.; Ripka, W.; Matthews, D.; Pinkerton, A.; Bounaud, P.-Y.; Hopkins, S. RAF Kinase Inhibitors. WO2011085269(A1), 2011.

(25) Tan, J.; Chen, Y.; Li, H.; Yasuda, N. Suzuki-Miyaura CrossCoupling Reactions of Unprotected Haloimidazoles. J. Org. Chem. 2014, 79 (18), 8871-8876.

(26) Rivara, M.; Zuliani, V. In Vivo Screening of Diarylimidazoles as Anticonvulsant Agents. Med. Chem. Res. 2012, 21 (11), 3428-3434.

(27) Bellina, F.; Cauteruccio, S.; Di Fiore, A.; Marchetti, C.; Rossi, R. Highly Selective Synthesis of 4(5)-Aryl-, 2,4(5)-Diaryl-, and 4,5Diaryl-1H-Imidazoles via Pd-Catalyzed Direct C-5 Arylation of 1Benzyl-1H-Imidazole. Tetrahedron 2008, 64 (26), 6060-6072.

(28) Chen, X. Y.; Englert, U.; Bolm, C. Base-Mediated Syntheses of Di- and Trisubstituted Imidazoles from Amidine Hydrochlorides and Bromoacetylenes. Chem. - Eur. J. 2015, 21 (38), 13221-13224.

(29) Bunge, K.; Huisgen, R.; Raab, R.; Sturm, H. J. 1.3-Dipolare Cycloadditionen, 64. Weitere Umsetzungen von Nitril-Yliden Mit Hetero-Mehrfachbindungen. Chem. Ber. 1972, 105 (4), 1307-1323.

(30) Shi, S.; Xu, K.; Jiang, C.; Ding, Z. ZnCl 2 -Catalyzed [3 + 2] Cycloaddition of Benzimidates and $2 \mathrm{H}$-Azirines for the Synthesis of Imidazoles. J. Org. Chem. 2018, 83 (23), 14791-14796.

(31) Neunhoeffer, H.; Lehmann, B.; Ewald, H. Zur Chemie Der 1,2,4-Triazine, VIII. Struktur Eines Reaktionsproduktes von 3-(PTolyl)-1,2,4-triazinen Mit Acetylendicarbonsäure-dimethylester. Justus Liebigs Ann. Chem. 1977, 1977 (9), 1421-1428.

(32) Bellina, F.; Cauteruccio, S.; Rossi, R. Efficient and Practical Synthesis of 4(5)-Aryl-1 H -Imidazoles and 2,4(5)-Diaryl-1 H -Imidazoles via Highly Selective Palladium-Catalyzed Arylation Reactions. J. Org. Chem. 2007, 72 (22), 8543-8546.

(33) Shimbayashi, T.; Okamoto, K.; Ohe, K. Synthesis of Imidazoles and Pyrimidines Using Palladium-Catalyzed Decarboxylative Intramolecular Condensation of 1,2,4-Oxadiazol-5(4 H)-Ones. Synlett 2014, 25 (13), 1916-1920.

(34) Zuliani, V.; Fantini, M.; Nigam, A.; Stables, J. P.; Patel, M. K.; Rivara, M. Anticonvulsant Activity of 2,4(1H)-Diarylimidazoles in Mice and Rats Acute Seizure Models. Bioorg. Med. Chem. 2010, 18 (22), 7957-7965.

(35) Rivara, M.; Baheti, A. R.; Fantini, M.; Cocconcelli, G.; Ghiron, C.; Kalmar, C. L.; Singh, N.; Merrick, E. C.; Patel, M. K.; Zuliani, V. 2,4(5)-Diarylimidazoles: Synthesis and Biological Evaluation of a New Class of Sodium Channel Blockers against HNav1.2. Bioorg. Med. Chem. Lett. 2008, 18 (20), 5460-5462.

(36) Donohoe, T. J.; Kabeshov, M. A.; Rathi, A. H.; Smith, I. E. D. Direct Preparation of Thiazoles, Imidazoles, Imidazopyridines and Thiazolidines from Alkenes. Org. Biomol. Chem. 2012, 10 (5), 10931101.

(37) Li, J.; Zhang, P.; Jiang, M.; Yang, H.; Zhao, Y.; Fu, H. Visible Light as a Sole Requirement for Intramolecular C(Sp3)-H Imination. Org. Lett. 2017, 19 (8), 1994-1997.

(38) Nery, M.; Azevedo, M.; Cardoso, J.; Slana, G.; Lopes, R.; Lopes, C. A New Chemoselective Synthesis of Brombuterol. Synthesis 2007, 2007 (10), 1471-1474.

(39) Taylor, N. J.; Emer, E.; Preshlock, S.; Schedler, M.; Tredwell, M.; Verhoog, S.; Mercier, J.; Genicot, C.; Gouverneur, V. Derisking the Cu-Mediated 18F-Fluorination of Heterocyclic Positron Emission
Tomography Radioligands. J. Am. Chem. Soc. 2017, 139 (24), 82678276.

(40) Vidadala, R. S. R.; Rivas, K. L.; Ojo, K. K.; Hulverson, M. A.; Zambriski, J. A.; Bruzual, I.; Schultz, T. L.; Huang, W.; Zhang, Z.; Scheele, S.; et al. Development of an Orally Available and Central Nervous System (CNS) Penetrant Toxoplasma Gondii CalciumDependent Protein Kinase 1 (TgCDPK1) Inhibitor with Minimal Human Ether-a-Go-Go-Related Gene (HERG) Activity for the Treatment of Toxoplasmosis. J. Med. Chem. 2016, 59 (13), 65316546.

(41) Naumiec, G. R.; Cai, L.; Lu, S.; Pike, V. W. Quinuclidine and DABCO Enhance the Radiofluorination of 5-Substituted 2-Halopyridines. Eur. J. Org. Chem. 2017, 2017 (45), 6593-6603. 